Supporting Information for

\title{
Efficient Functionalizations of Heteroatom Bridged Calix[2]arene[2]triazines on the Larger Rim
}

\author{
Hai-Bo Yang, De-Xian Wang,* Qi-Qiang Wang, Mei-Xiang Wang*
}

Beijing National Laboratory for Molecular Sciences, Laboratory of Chemical Biology, Institute of Chemistry, Chinese Academy of Sciences, Beijing, 100080, China

\section{Table of Contents}

Copies of ${ }^{1} \mathrm{H}$ and ${ }^{13} \mathrm{C}$ NMR Spectra for 6-9, 12a-e, 13a-c

UV-vis Titration of 12a-c with $\mathrm{Cu}^{2+}, \mathrm{Fe}^{2+}, \mathrm{Co}^{2+}, \mathrm{Ni}^{2+}, \mathrm{Zn}^{2+}$ and 13a-c with $\mathrm{Cu}^{2+}$

ESI-MS of the Complexs 12a-c with $\mathrm{Cu}\left(\mathrm{ClO}_{4}\right)_{2}$ 48-53 


\section{${ }^{1} \mathrm{H}$ NMR of 6}

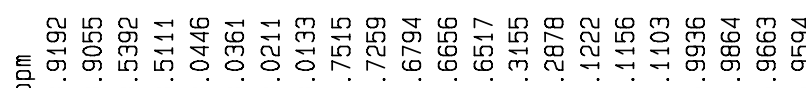

ம ம
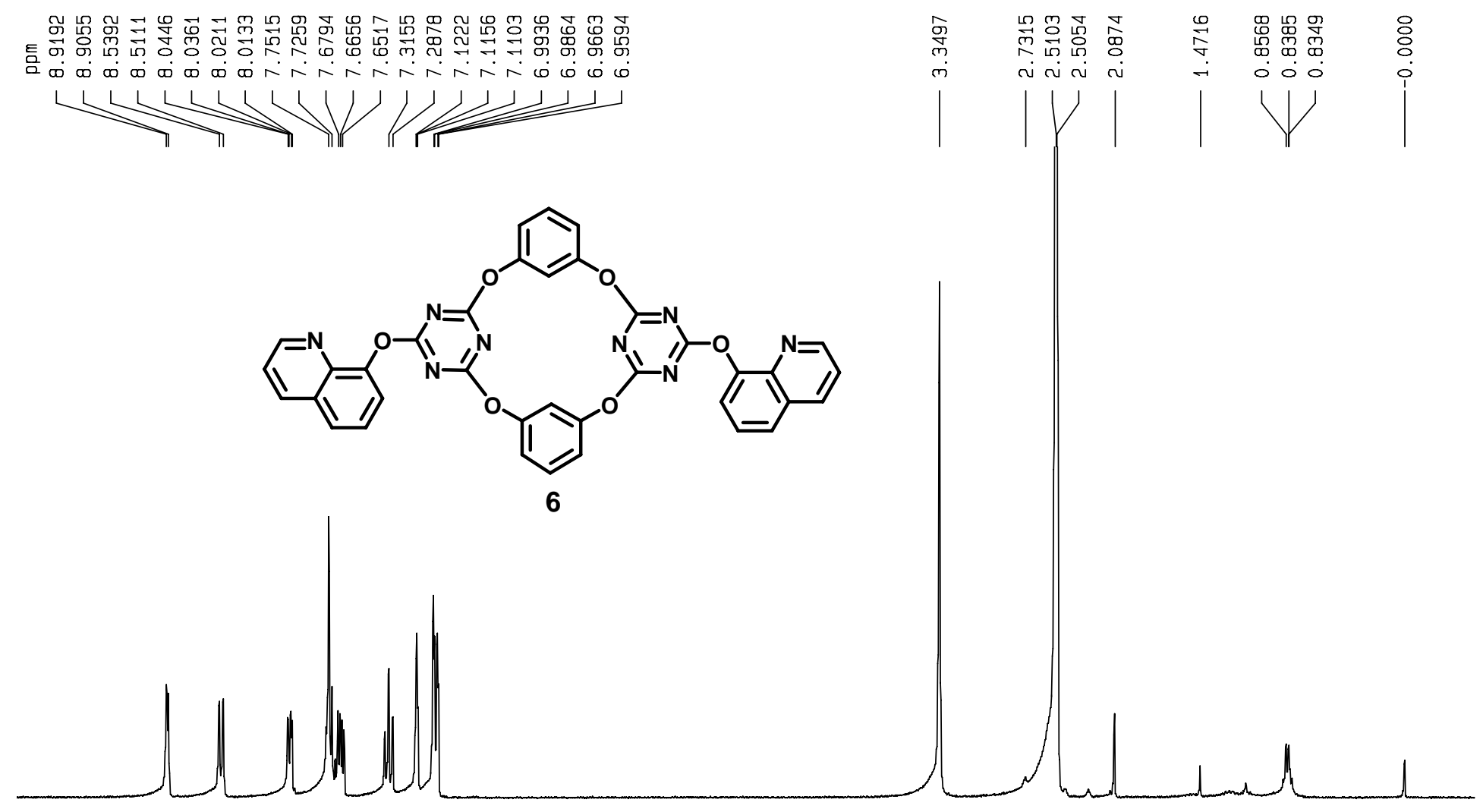

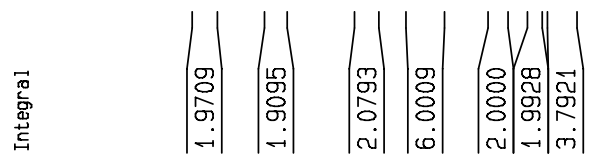

ppm
1
8

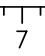

Current Data Parameters NAME $\quad$ yhb-1106-408a

EXPONO

F2 - Acquisition Parameters

Date $\quad 20041107$

$\begin{array}{lr}20041107 \\ \text { Time } & 6.03\end{array}$

INSTRUM av300 PROBHD $5 \mathrm{~mm}$ DUL $13 \mathrm{C}-1$ $\begin{array}{lr}\text { PULPHOG } & 2930 \\ \text { TD } & 65536\end{array}$

SOLVENT DMSO

NS

SWH $\quad 6172.839 \mathrm{~Hz}$

FIDRES $\quad 0.094190 \mathrm{~Hz}$

AQ $\quad 5.3084660 \mathrm{sec}$

DW $\quad 81.000$ usec

81.000 usec
6.00 usec

$2.00000000 \mathrm{sec}$

$=======$ CHANNEL $f 1=======$
NUC1 $1 \mathrm{H}$

P1 9.30 usec

SF01 $\quad 300.1318534 \mathrm{MHz}$

2 - Processing parameters

SI 32768

$\mathrm{SF} \quad 300.1299991 \mathrm{MHz}$

WDW EM

$\begin{array}{lc}\text { LB } & 0 \\ \text { GB } & 0.30 \mathrm{~Hz}\end{array}$

10 NMB plot parameters

CX $22.00 \mathrm{~cm}$

$\begin{array}{lll}\text { CY } & 20.00 \mathrm{~cm} \\ \text { CY } & 3001.30 \mathrm{cz}\end{array}$

F1 $\quad 10.000 \mathrm{ppm}$

$\begin{array}{ll}\text { F1 } & 3001.30 \mathrm{~Hz} \\ \text { F2P } & -0.500 \mathrm{ppm}\end{array}$

$\begin{array}{lll}\text { F2 } & -150.06 \mathrm{~Hz} \\ \text { PPMCM } & 0.47727 \mathrm{PPm} / \mathrm{Cm}\end{array}$

$\begin{array}{lr}\text { HZCM } & 0.47727 \mathrm{ppm} / \mathrm{cm} \\ & 143.24385 \mathrm{~Hz} / \mathrm{cm}\end{array}$ 

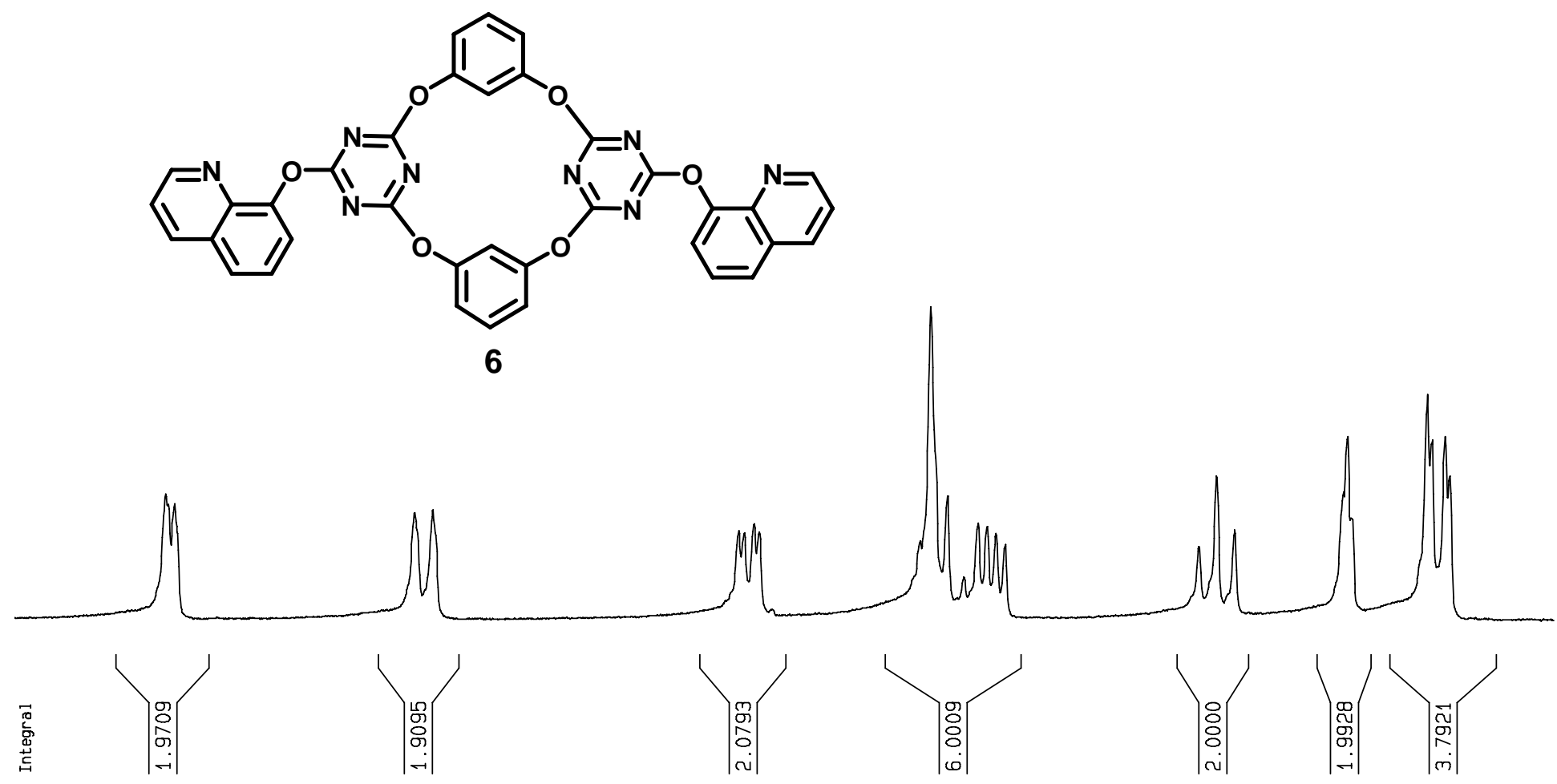

Current Data Parameters NAME $\quad y n b-1106-408 a$ EXPNO

F2 - Acquisition Parameter

Date

Date 20041107 PROBHD $5 \mathrm{~mm}$ DUL $13 \mathrm{C}-1$ PULPROG Zg30 TD $\quad 65536$
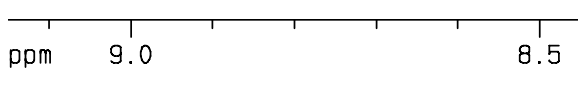

8.0

7.5 
${ }^{13} \mathrm{C}$ NMR of 6

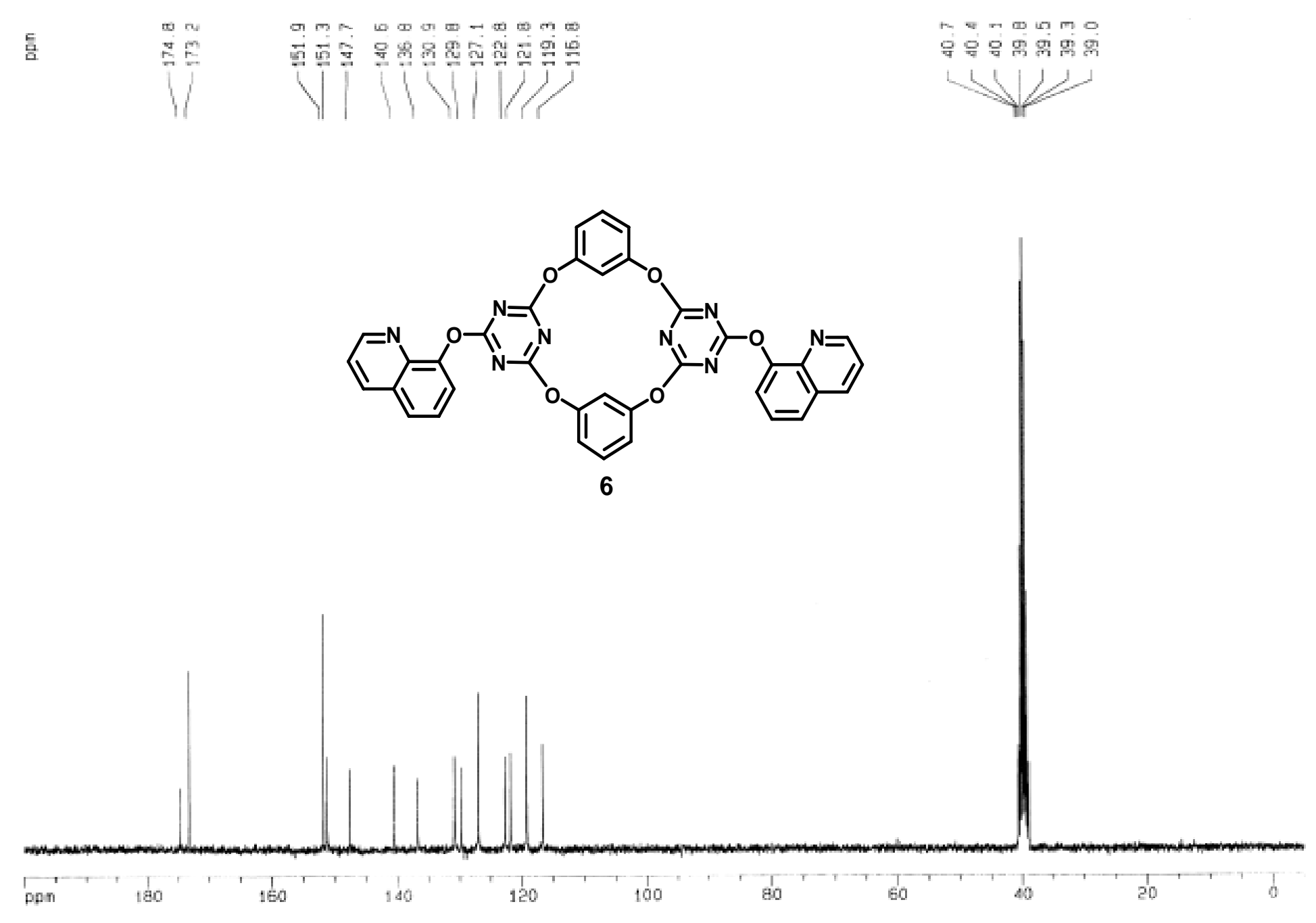

\begin{tabular}{|c|c|c|}
\hline \multicolumn{3}{|c|}{ FZ - Acquisition Paraneters } \\
\hline Date_ & 20020124 & \\
\hline Tune & 3.07 & \\
\hline INGTFUM & $07 \times 300$ & \\
\hline Рคавн & 881 & \\
\hline PUL.FAOG & 2gdc & \\
\hline то & 32768 & \\
\hline SOLVENT & OMSO & \\
\hline N5 & 1550 & \\
\hline 05 & 2 & \\
\hline 5NH & 17361.111 & \\
\hline FIDFES & 0.529819 & \\
\hline $\mathrm{AD}$ & 0.9437664 & $\sec$ \\
\hline м6 & 256 & \\
\hline ס & 28.000 & usec \\
\hline DE & 4.50 & usec \\
\hline TE & 300.0 & \\
\hline D11 & 0.03000000 & sec \\
\hline PL12 & 26.00 & 08 \\
\hline CPOPAG: & valtz16 & \\
\hline PCPDE & 100.00 & user \\
\hline SFa2 & 300.1310035 & N-2 \\
\hline neuce & 211 & \\
\hline PL2 & 2.00 & \\
\hline D1 & 2.00000000 & ser \\
\hline$p_{1}$ & 6.50 & usec \\
\hline SFos & 75.47556600 & $\mathrm{MHI}$ \\
\hline NuC1 & $13 \mathrm{C}$ & \\
\hline PLI & 0.00 & \\
\hline \multirow{2}{*}{\multicolumn{3}{|c|}{$\begin{array}{l}\text { F2 - Processing Daransters } \\
\text { SI }\end{array}$}} \\
\hline & & \\
\hline SF & 75.4677658 & NH2 \\
\hline KDW & EM & \\
\hline SSB & 0 & \\
\hline LB & 1.00 & \\
\hline 68 & 0 & \\
\hline$P C$ & 0.10 & \\
\hline \multicolumn{3}{|c|}{ 10 NWF plot paraneters } \\
\hline$[x$ & 21.50 & \\
\hline$F$ 1P & 200.000 & pon \\
\hline$F_{1}$ & 15093.55 & $\mathrm{~Hz}$ \\
\hline$F \mathfrak{P a}$ & -5.000 & ppn \\
\hline F2 & -377.34 & \\
\hline $\mathrm{PPQCM}$ & 9. 53488 & ppen/can \\
\hline $\mathrm{HzCM}$ & 719.57629 & $\mathrm{~Hz} / \mathrm{Ca}$ \\
\hline
\end{tabular}


${ }^{1} \mathrm{H}$ NMR of 7
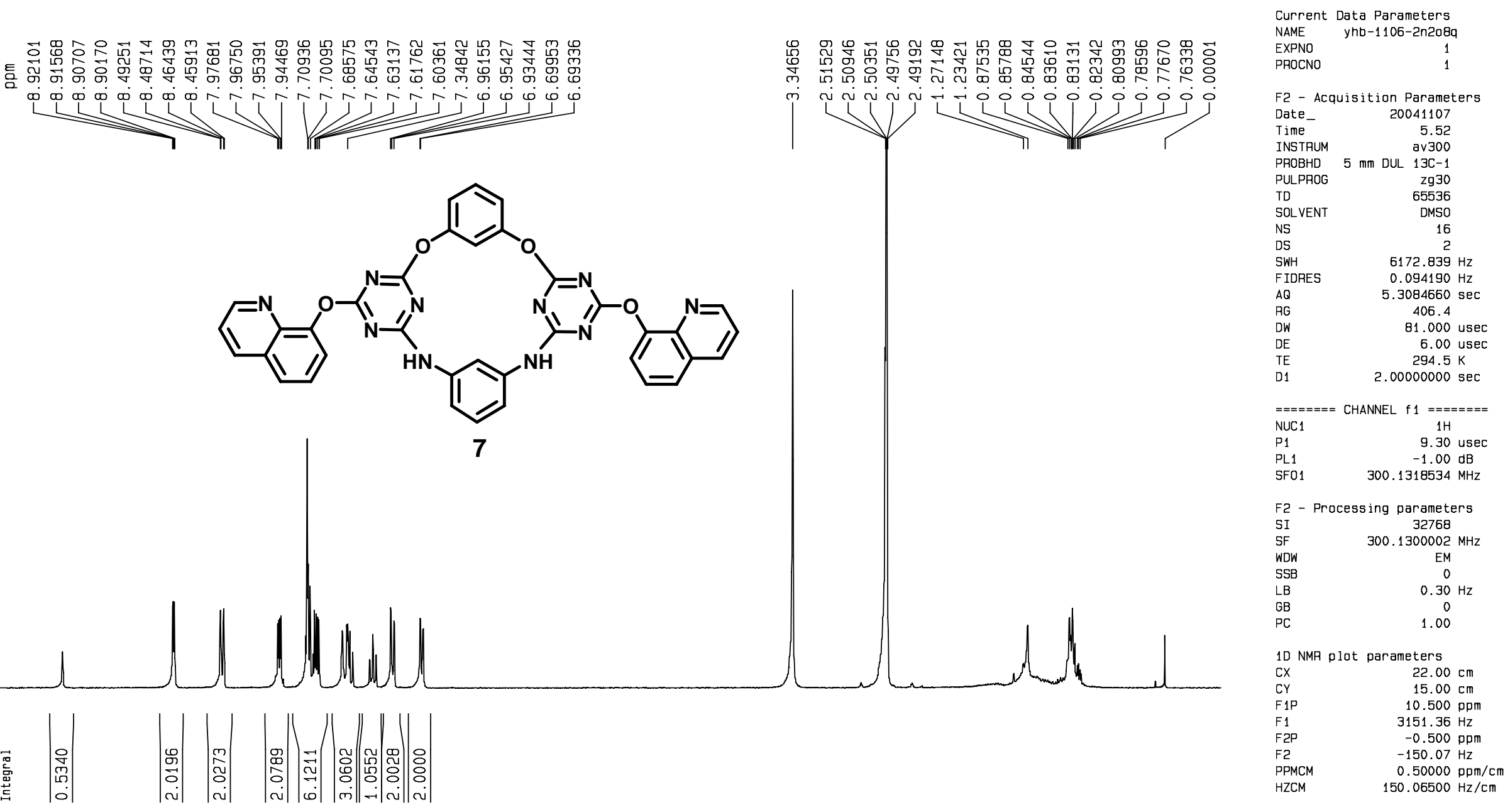

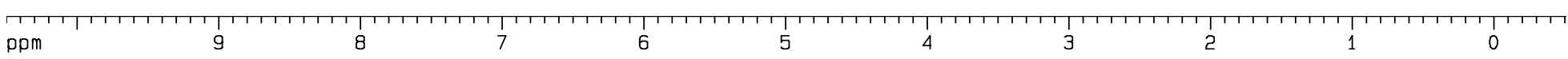




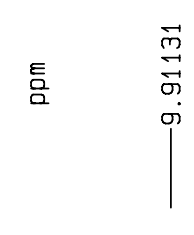

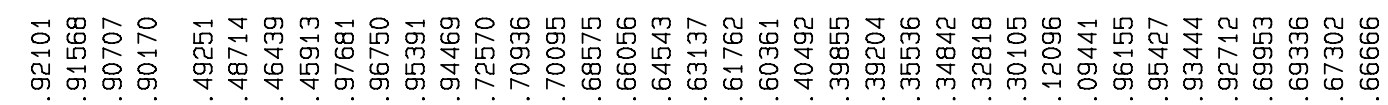

i
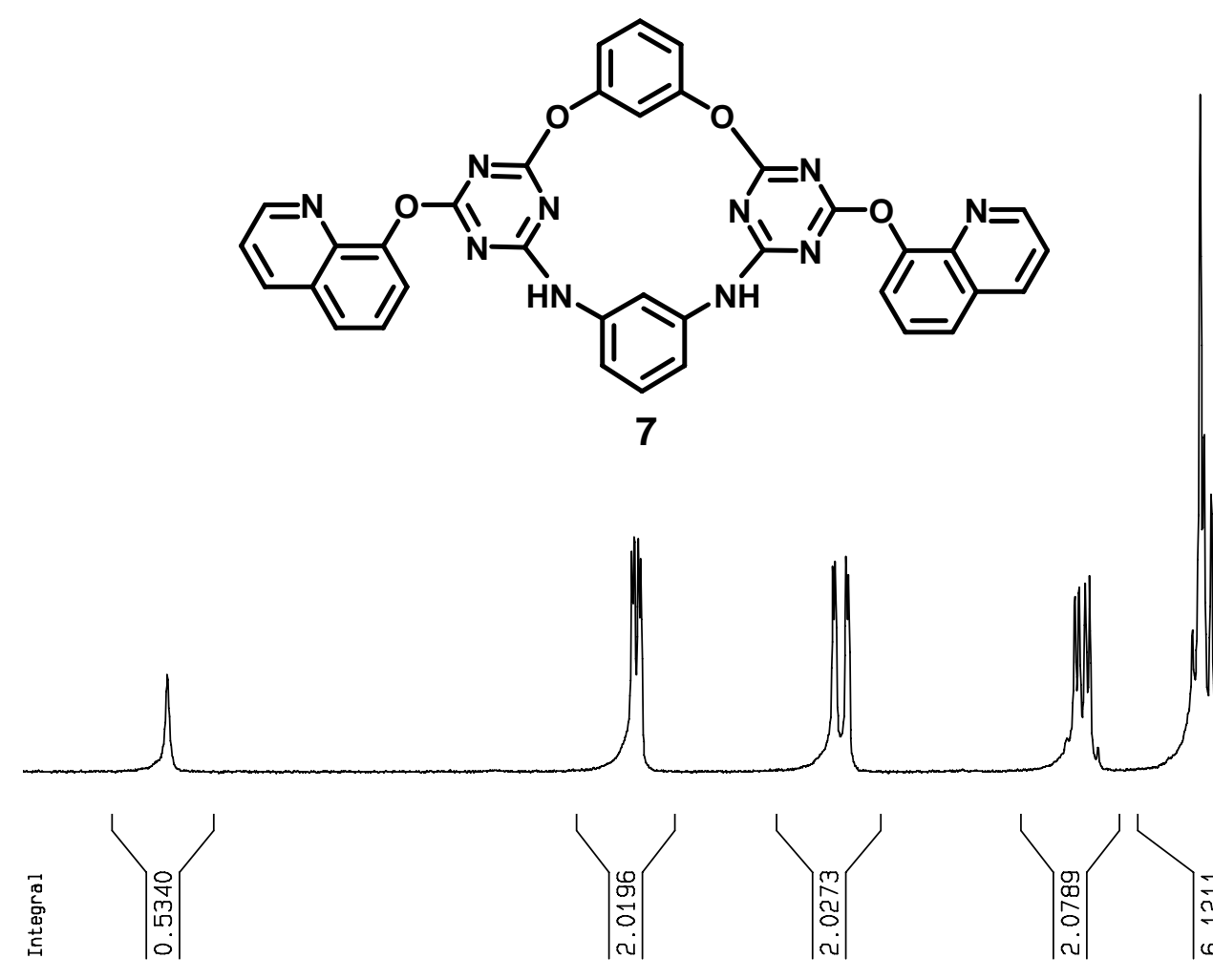

$\begin{array}{ll} & \\ \text { ppm } & 10.0\end{array}$

9.5

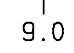

8.5

8.0

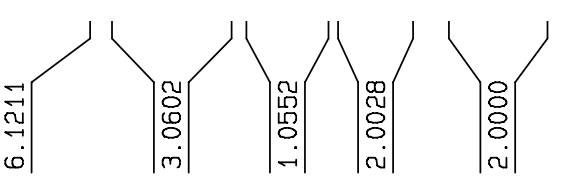

9.0

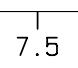

7.0

1.0

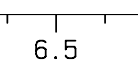

Current Data Parameters

NAME $\quad y n b-1106-2 n 208$

EXPNO

F2 - Acquisition Parameters

Date

$\begin{array}{lr}\text { Time } & 5.52 \\ \text { INSTRUM } & \text { av300 }\end{array}$ 20041107

PROBHD $5 \mathrm{~mm}$ DUL $13 \mathrm{C}-1$

2930

$\begin{array}{lr}\text { TD } & 65536 \\ \text { SOLVENT } & \text { DMSO }\end{array}$

$\begin{array}{lr}\text { SOLVENT } & \text { DMSO } \\ \text { NS } & 16 \\ \text { DS } & 2\end{array}$

SWH $\quad 6172.839 \mathrm{~Hz}$

$\begin{array}{ll}0.094190 \mathrm{~Hz} \\ \text { AQ } & 5.3084660 \mathrm{sec}\end{array}$

RG $\quad 406.4$

$\begin{array}{lr}\text { DW } & 81.000 \text { usec } \\ \text { DE } & 6.00 \text { usec }\end{array}$

TE $294.5 \mathrm{~K}$

Di

$=======$ CHANNEL $f 1======$

NUC1

P1 9.30 usec

$\begin{array}{lr}\text { PL1 } & -1.00 \mathrm{~dB} \\ \text { SF01 } & 300.1318534 \mathrm{MHz}\end{array}$

F2 - Processing parameters

$\begin{array}{lc}\text { SI } & 32768 \\ \text { SF } & 300.1300002 \mathrm{MHZ}\end{array}$

WOW
$55 B$

LB $\quad 0.30 \mathrm{~Hz}$

$\begin{array}{lr}\text { GB } & 0 \\ P C & 1.00\end{array}$

10 NMR plot parameters

CX $\quad 22.00 \mathrm{~cm}$

$\begin{array}{ll}\text { CY } & 25.00 \mathrm{~cm} \\ \text { FIP } & 10.221 \mathrm{~cm}\end{array}$

$\begin{array}{ll}\text { F1P } & 10.221 \mathrm{ppm} \\ \text { F1 } & 3067.78 \mathrm{~Hz}\end{array}$

$\begin{array}{lr}\text { F1 } & 1067.78 \mathrm{~Hz} \\ \text { F2P } & 6.310 \mathrm{ppm} \\ \text { F2 } & 1893.91 \mathrm{~Hz}\end{array}$

$\begin{array}{ll}\text { F2 } & 1893.91 \mathrm{~Hz} \\ \text { PPMCM } & 0.17778 \mathrm{pDm} / \mathrm{Cm}\end{array}$

$\begin{array}{lr}\text { PPMCM } & 0.17778 \mathrm{pDm} / \mathrm{Cm} \\ \mathrm{HZCM} & 53.35778 \mathrm{~Hz} / \mathrm{cm}\end{array}$ 
${ }^{13} \mathrm{C}$ NMR of 7

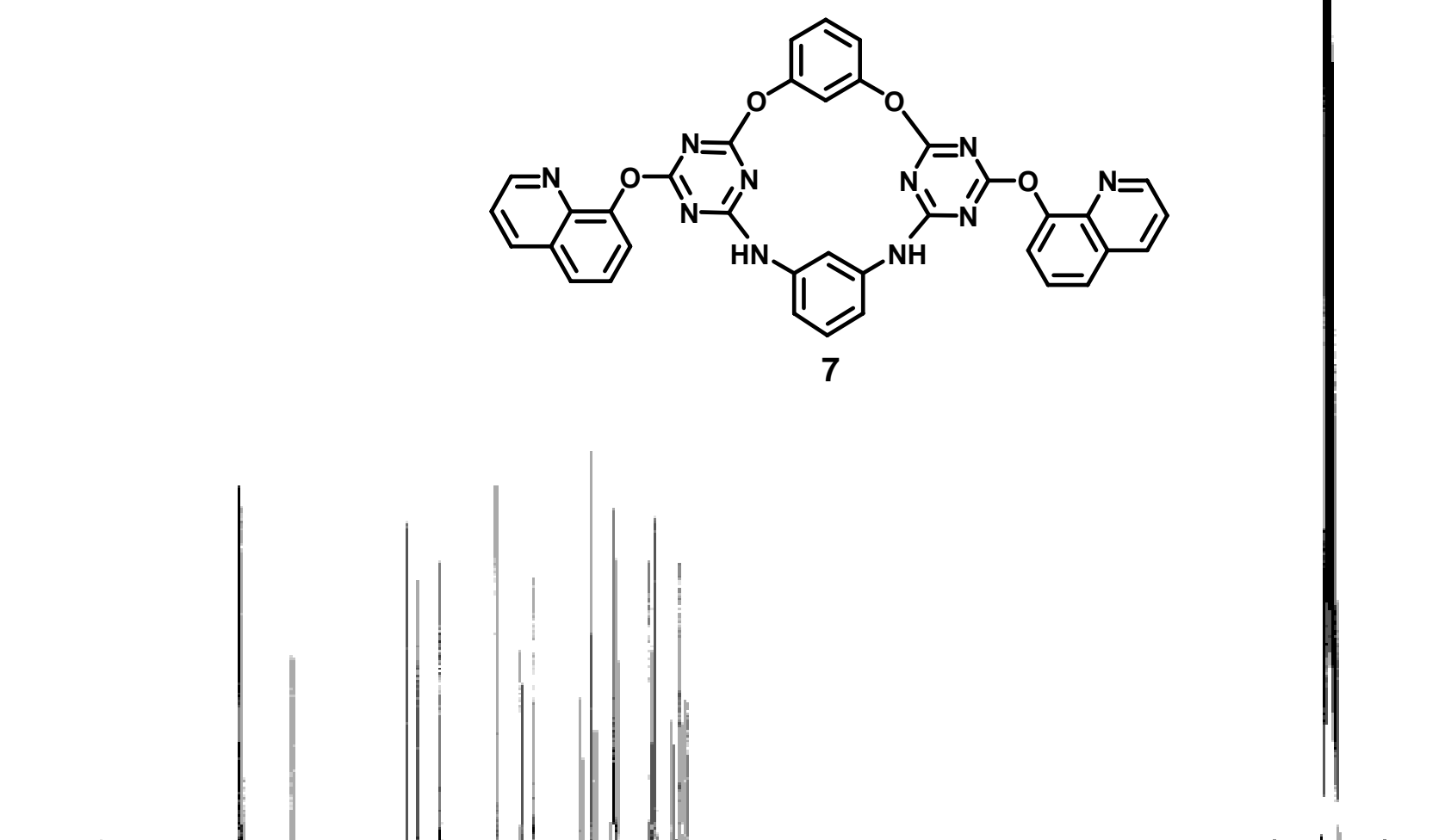

F2 - Acquisition Farenete"s

Date_ 20020129

Time 7.09

$\begin{array}{lc}\text { INSTEAN } & \text { anx } 300 \\ \text { SADEHO } & \text { EeI }\end{array}$

QULPFOG 2 gde

32768
SDLVENT DNSO

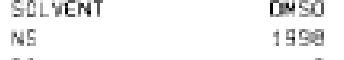

CWH $17961.111 \mathrm{HL}$

FILPES $\quad 0.529819 \mathrm{HC}$

AQ O. 9437684 sec

DK 28.800 used

OE 4.50 ves

$011 \quad 0.03000000 \mathrm{ser}$

PLI3 13

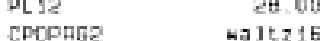

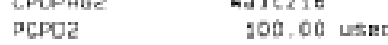

SFOP $360-1310025 \mathrm{NH}^{2}$

Nuce

noce

19. 20000000 여

P. 6.50 use

SF01 $75.4755660 \mathrm{~N}-2$

Nuc1 $13 \mathrm{C}$

-2 - Processirn paraneters

SI B192

SF $\quad 75.4677522 \mathrm{MHz}$

NDW

$\begin{array}{ll}S S 9 & 0 \\ -B & 1.00 \mathrm{~Hz}\end{array}$

$\begin{array}{ll}\mathrm{GB} & 0.10\end{array}$

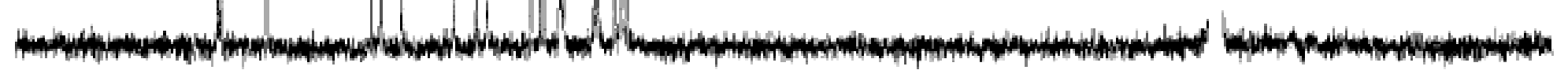

10 NHF olot paraneters

Cx 21.50 tm

Fi 200000000

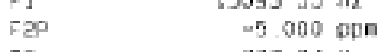

$72 \quad-37734 \mathrm{~Hz}$

IPNCM $\quad 953488 \mathrm{ppm} / \mathrm{cm}$

60

60

40

20 
${ }^{1} \mathrm{H}$ NMR of 8

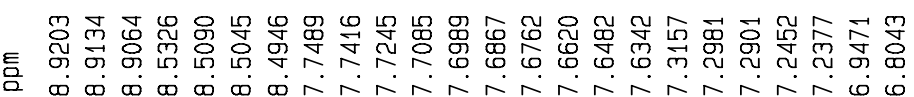
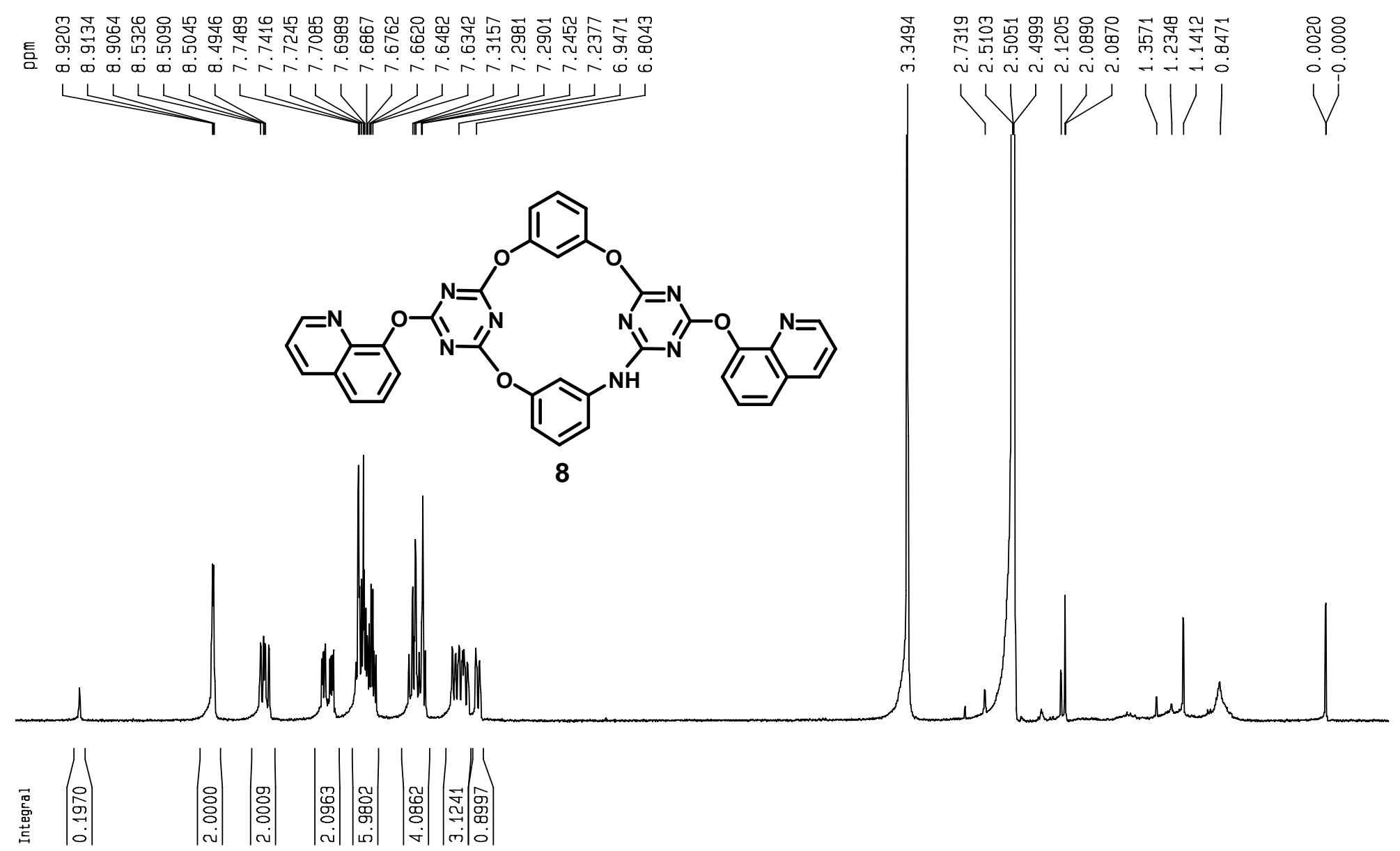

Current Data Parameters

VAME Yhb-1106-301n8a

TROCNO

$=2$ - Acquisition Parameters

Jate

$\begin{array}{lr} & -20041107 \\ \text { Jate } & 5.13 \\ \text { Time } & \\ \text { INSTRUM } & \text { av300 }\end{array}$

ЭАOBHD $5 \mathrm{~mm}$ DUL $13 \mathrm{C}-1$

JULPROG Zg30

SOLVENT DMSO

JS

SWH $\quad 6172.839 \mathrm{~Hz}$

$\begin{array}{lll} & 0.094190 \mathrm{~Hz} \\ A Q & -309650 \mathrm{seC}\end{array}$

ZG $\quad 5.3084660 \mathrm{sec}$

JW $\quad \begin{aligned} 322.5 \\ \text { JW }\end{aligned}$

TE $\quad 6.00$ use

$2.00000000 \mathrm{sec}$

$=======$ CHANNEL $f 1=======$
VUC1 $1 \mathrm{H}$

9.30 use

SF01 $\quad-1.00 \mathrm{~dB}$

=2 - Processing parameters

SI 32768

SF $300.1299998 \mathrm{MHz}$

$\begin{array}{lc}\text { WDW } & E M \\ \text { SSB } & 0 \\ -B & 0.30 \mathrm{~Hz}\end{array}$

${ }^{3}$

10 NMF plot parameters

cX $\quad 22.00 \mathrm{~cm}$

$\begin{array}{lr}\text { TY } & 35.00 \mathrm{~cm} \\ =1 \mathrm{P} & 10.500 \mathrm{ppm} \\ =1 & 3151.36 \mathrm{~Hz}\end{array}$

$\begin{array}{ll}-2 \mathrm{P} & -0.500 \mathrm{ppm} \\ =-2 & -150.07 \mathrm{~Hz}\end{array}$

JPMCM $\quad-150.07 \mathrm{~Hz}$

$\begin{array}{ll} & 0.50000 \mathrm{ppm} / \mathrm{cm} \\ \text { HZCM } & 150.06500 \mathrm{~Hz} / \mathrm{cm}\end{array}$

(11

9

8

7

6

5

4

3

2 


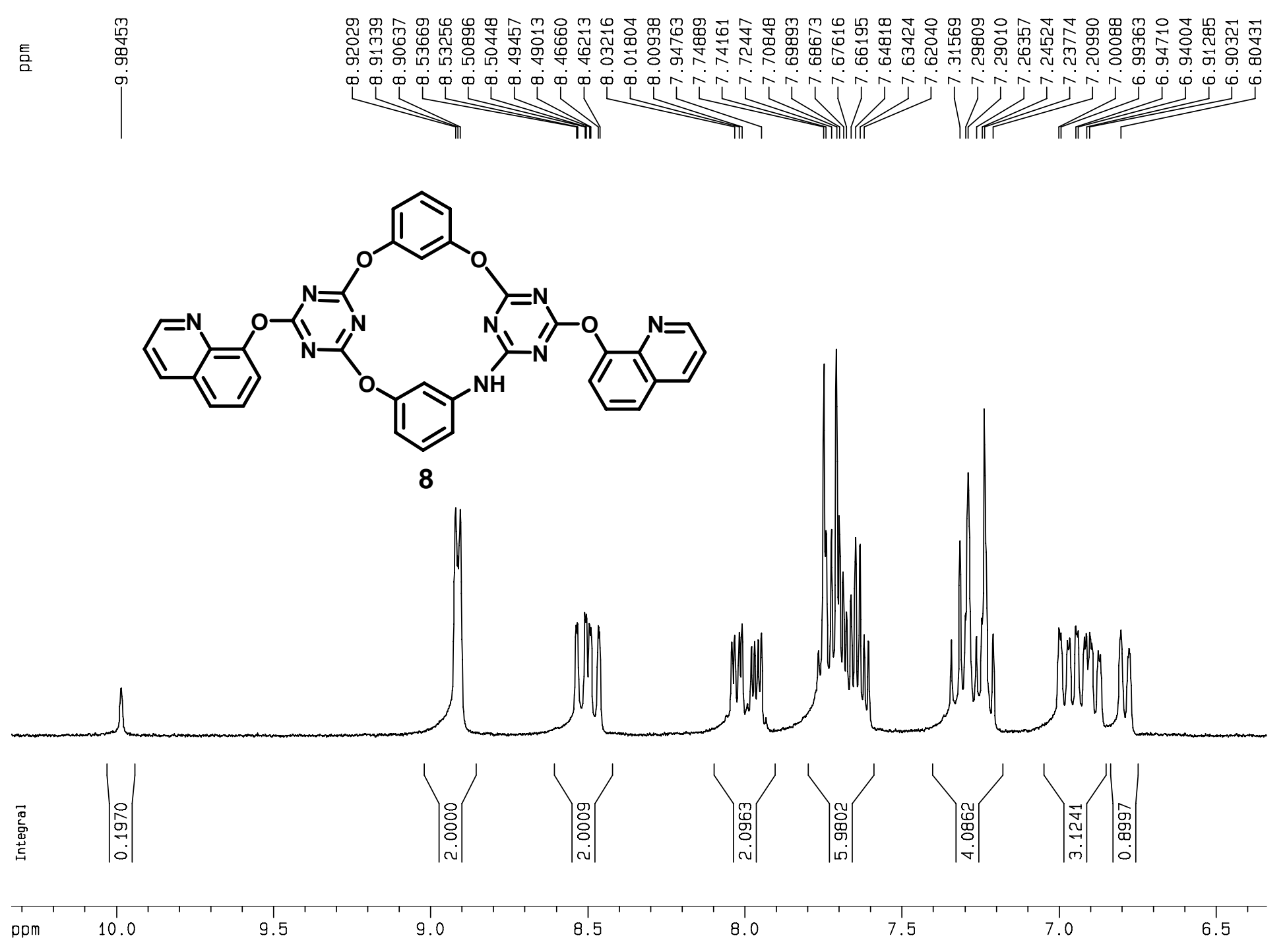

Current Data Parameters

NAME $\quad$ yhb-1106-301n8q EXPNO
PROCNO

F2 - Acquisition Parameters

Date_ $\quad 20041107$

Time

INSTRUM av300

$5 \mathrm{~mm}$ DUL $13 \mathrm{C}-1$

\begin{tabular}{lr} 
PULPROG & gg 30 \\
TD & 65536 \\
\hline
\end{tabular}

SOLVENT

NS
DS

DS
SWH

FIDRES $\quad 0.094190 \mathrm{~Hz}$

AQ $\quad 5.3084660 \mathrm{sec}$

RG $\quad 322.5$

DW $\quad 81.000$ usec

DE 6.00 use

$\begin{array}{lr}\text { TE } & 294.2 \mathrm{~K} \\ \text { D1 } & 2.00000000 \mathrm{sec}\end{array}$

$======$ CHANNEL $f 1$
NUC1

NUC1 $1 \mathrm{H}$

$\begin{array}{lr}\text { PL1 } & -1.00 \mathrm{~dB}\end{array}$

5F01 $300.1318534 \mathrm{MHz}$

F2 - Processing parameters

SI 32768

$\mathrm{SF} \quad 300.1299998 \mathrm{MHz}$

$\begin{array}{lr}\text { WDW } & \text { EM } \\ \text { SSB } & 0\end{array}$

$\begin{array}{ll}S S B & 0 \\ L B & 0.30 \mathrm{~Hz}\end{array}$

$\begin{array}{lr}\text { GB } & 0 \\ \mathrm{PC} & 1.00\end{array}$

10 NMR plot parameters

CX

$\begin{array}{ll}\text { CX } & 22.00 \mathrm{~cm} \\ \text { CY } & 50.00 \mathrm{~cm}\end{array}$

FIP $\quad 10.334 \mathrm{ppm}$

$\begin{array}{ll}F 1 & 3101.58 \mathrm{~Hz}\end{array}$

$\begin{array}{ll} & 6.340 \mathrm{ppm} \\ \mathrm{F} & \\ \mathrm{F} & 1902.69 \mathrm{~Hz}\end{array}$

PPMCM $\quad 0.18157 \mathrm{ppm} / \mathrm{c}$

HZCM $\quad 54.49490 \mathrm{~Hz} / \mathrm{cm}$ 
${ }^{13} \mathrm{C}$ NMR of 8

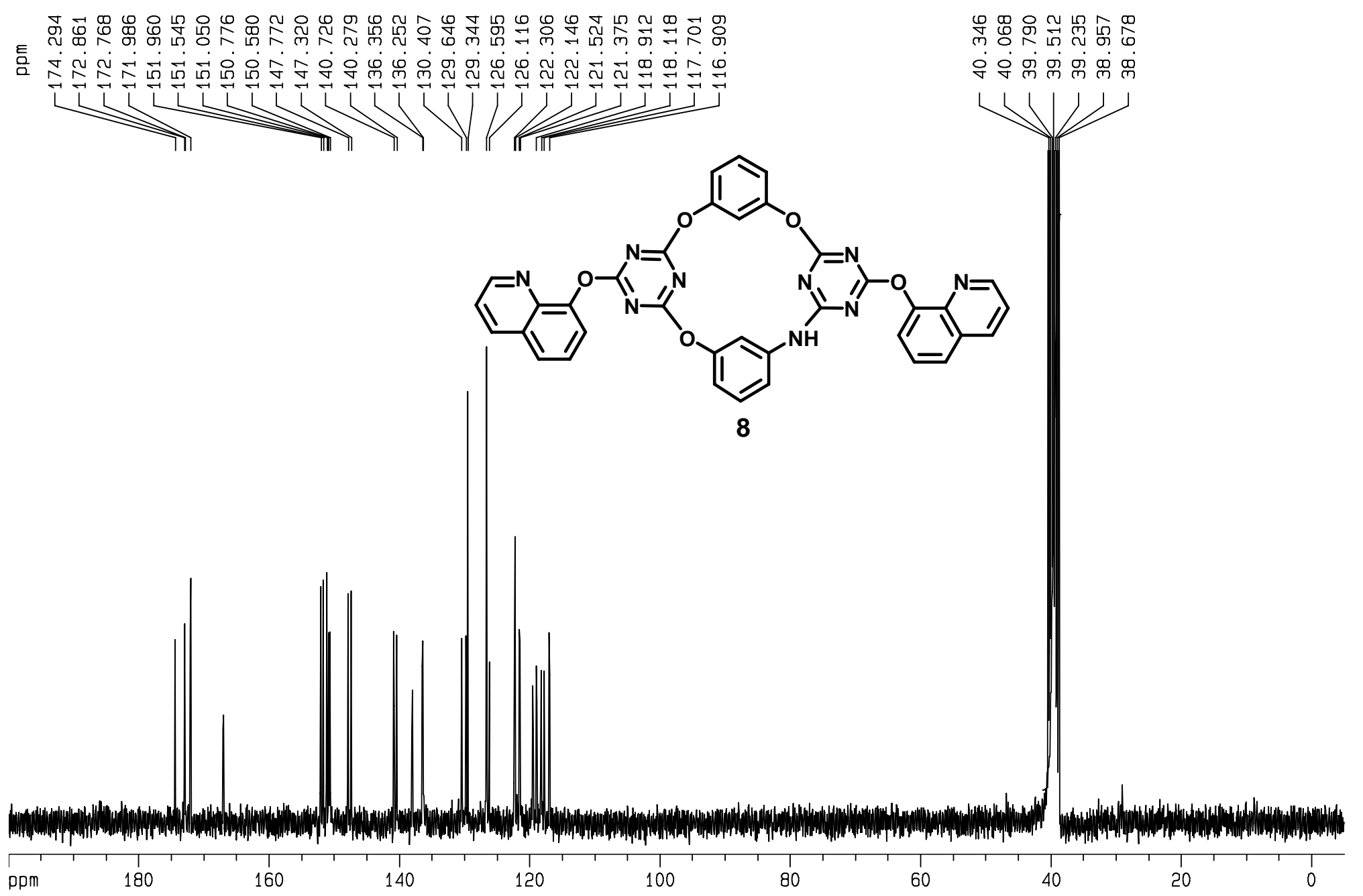

\begin{tabular}{|c|c|}
\hline & \\
\hline $\begin{array}{l}\text { PULPROG } \\
\text { TD }\end{array}$ & $\begin{array}{r}\text { zgpg30 } \\
65536\end{array}$ \\
\hline SOLVENT & \\
\hline NS & 2788 \\
\hline DS & 4 \\
\hline SWH & $17985.611 \mathrm{~Hz}$ \\
\hline FIDRES & $0.274439 \mathrm{~Hz}$ \\
\hline$A Q$ & $1.8219508 \mathrm{sec}$ \\
\hline $\mathrm{RG}$ & 287.4 \\
\hline DW & 27.800 usec \\
\hline DE & 6.00 usec \\
\hline TE & $301.7 \mathrm{~K}$ \\
\hline D1 & $2.00000000 \mathrm{sec}$ \\
\hline d11 & $0.03000000 \mathrm{sec}$ \\
\hline d12 & $0.00002000 \mathrm{sec}$ \\
\hline$======$ & ANNEL $f 1$ ======= \\
\hline NUC1 & $13 \mathrm{C}$ \\
\hline P1 & 9.40 usec \\
\hline PL1 & $-1.00 \mathrm{~dB}$ \\
\hline SF01 & $75.4752953 \mathrm{MHZ}$ \\
\hline$======$ & ANNEL $f 2=======$ \\
\hline CPDPAG2 & waltz16 \\
\hline NUC2 & $1 \mathrm{H}$ \\
\hline PCPD2 & 80.00 usec \\
\hline PL2 & $-1.00 \mathrm{~dB}$ \\
\hline PL12 & $18.00 \mathrm{~dB}$ \\
\hline PL13 & $18.00 \mathrm{~dB}$ \\
\hline SF02 & $300.1312005 \mathrm{MHz}$ \\
\hline$F 2-\operatorname{Pr}$ & sing parameters \\
\hline SI & 32768 \\
\hline $\mathrm{SF}$ & $75.4677867 \mathrm{MHZ}$ \\
\hline WOW & EM \\
\hline SSB & 0 \\
\hline LB & $1.00 \mathrm{~Hz}$ \\
\hline GB & 0 \\
\hline PC & 1.40 \\
\hline 10 NMR & parameters \\
\hline$c x$ & $22.00 \mathrm{~cm}$ \\
\hline CY & $100.00 \mathrm{~cm}$ \\
\hline F1P & $200.000 \mathrm{ppm}$ \\
\hline F1 & $15093.56 \mathrm{~Hz}$ \\
\hline F2P & $-5.000 \mathrm{ppm}$ \\
\hline F2 & $-377.34 \mathrm{~Hz}$ \\
\hline PPMCM & $9.31818 \mathrm{ppm} / \mathrm{c}$ \\
\hline HZCM & $703.22253 \mathrm{H}$ \\
\hline
\end{tabular}




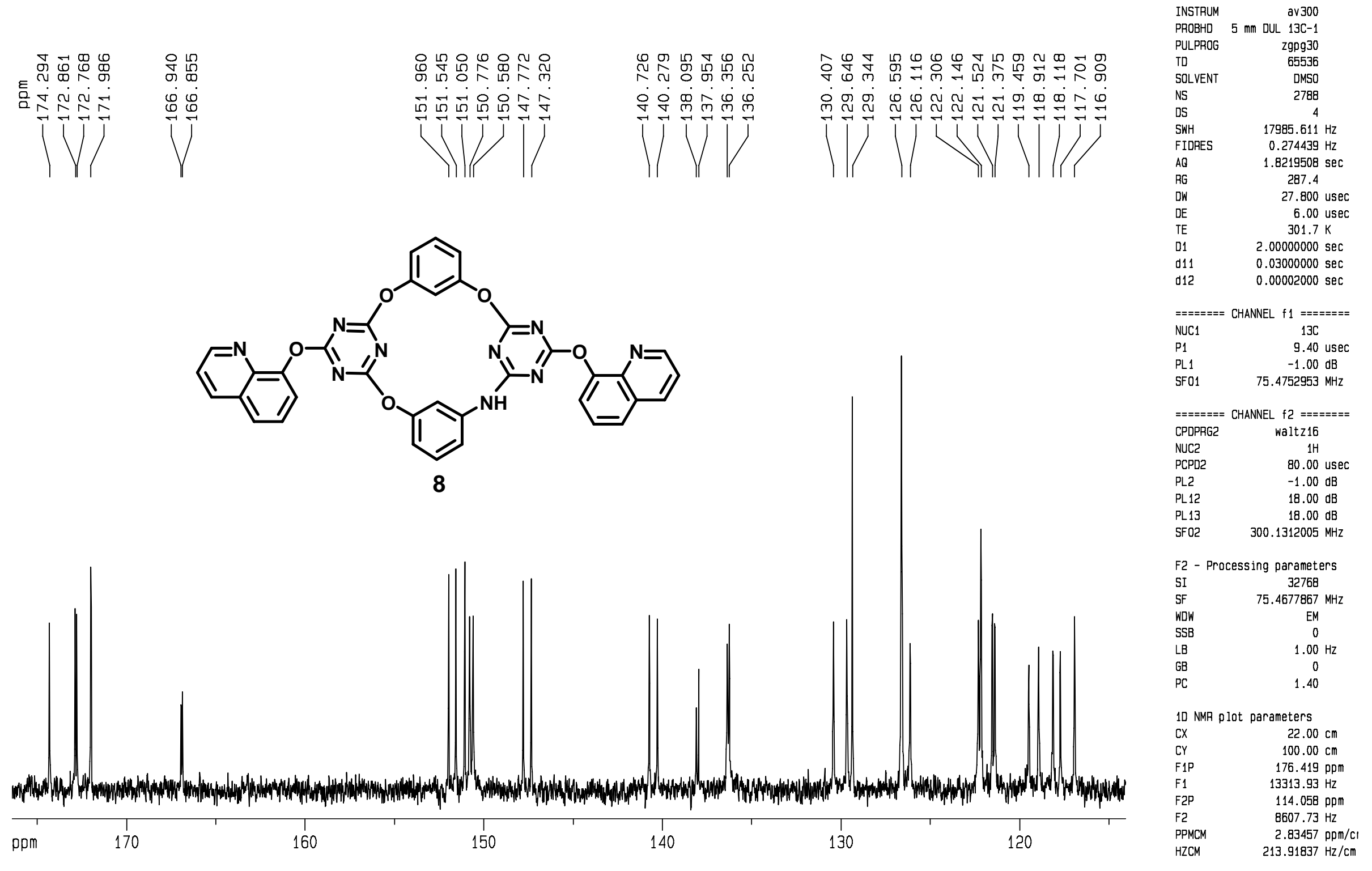




\section{${ }^{1}$ H NMR of 9}

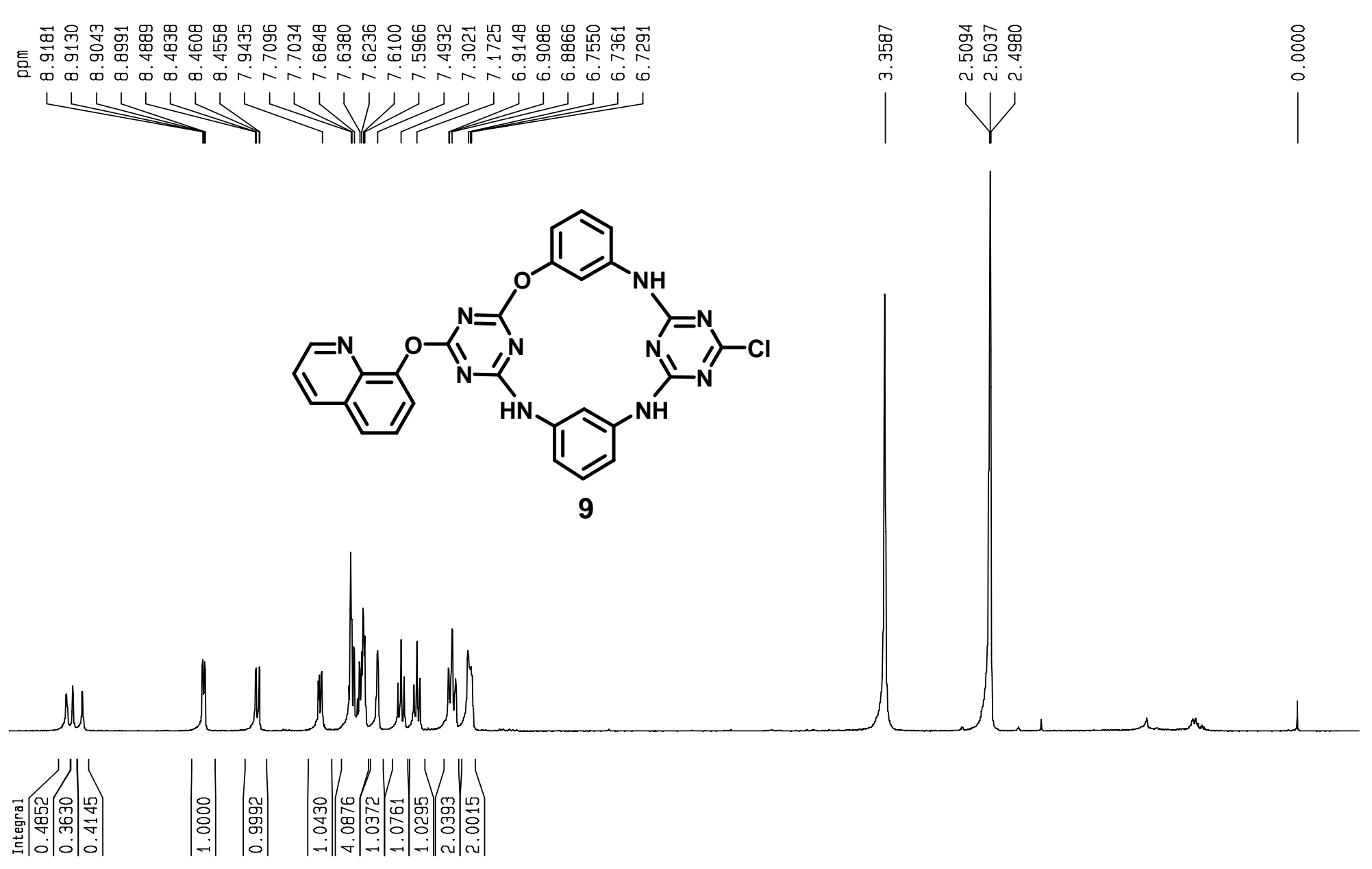

Current Data Parameters

VAME $\quad$ yhb-1106-3n108q

JROCNO

=2 - Acquisition Parameter

Jate_-

5.41
INSTRUM

गROBHD $5 \mathrm{~mm}$ DUL $13 \mathrm{C}-1$

JULPROG Z930

$\begin{array}{lr}\text { TD } & 65536 \\ \text { SDIVNT } & \text { DMSO }\end{array}$

$\begin{array}{lr}\text { VS } & 16 \\ \text { JS } & 5\end{array}$

JWH $\quad 6172.839 \mathrm{~Hz}$

$\begin{array}{ll}=\text { IDRES } & 0.094190 \mathrm{~Hz} \\ A Q & 5.3084660 \mathrm{sec}\end{array}$

7G 322.5

$\begin{array}{rr}\text { JW } & 81.000 \text { usec } \\ \text { JE } & 6.00 \text { usec }\end{array}$

$6.00 \mathrm{usec}$
$294.2 \mathrm{~K}$

J1 $2.00000000 \mathrm{sec}$

$=======$ CHANNEL $f 1$ $1=======$
VUC1 $1 \mathrm{H}$

ग1 9.30 usec

$\begin{array}{lr}\text { IL1 } & -1.00 \mathrm{~dB} \\ \text { SF01 } & 300.1318534 \mathrm{MHz}\end{array}$

$=2$ - Processing parameters

SI $\quad 32768$

WDW

$\begin{array}{lc}\text { WOW } & \text { EM } \\ \text { SSB } & 0 \\ B & 0.30 \mathrm{~Hz}\end{array}$

$\begin{array}{lr}\mathrm{AB} & 0.30 \\ \mathrm{~GB} & 0 \\ \mathrm{JC} & 1.00\end{array}$

10 NMA plot parameters

$\begin{array}{ll}\text { CX } & 22.00 \mathrm{~cm} \\ \mathrm{cY} & 10.00 \mathrm{~cm}\end{array}$

$\begin{array}{lll}=1 \mathrm{P} & 10.00 \mathrm{~cm} \\ = & 10.500 \mathrm{ppm}\end{array}$

$\begin{array}{ll}=1 & 3151.36 \mathrm{~Hz} \\ =2 \mathrm{p} & -0.500 \mathrm{ppm}\end{array}$

$-0.500 \mathrm{ppm}$
$-150.07 \mathrm{~Hz}$ $\begin{array}{lr}\text { JPMCM } & 0.50000 \mathrm{ppm} / \mathrm{cm} \\ \text { HZCM } & 150.06500 \mathrm{~Hz} / \mathrm{cm}\end{array}$

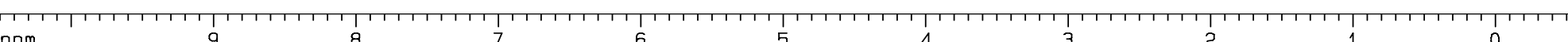



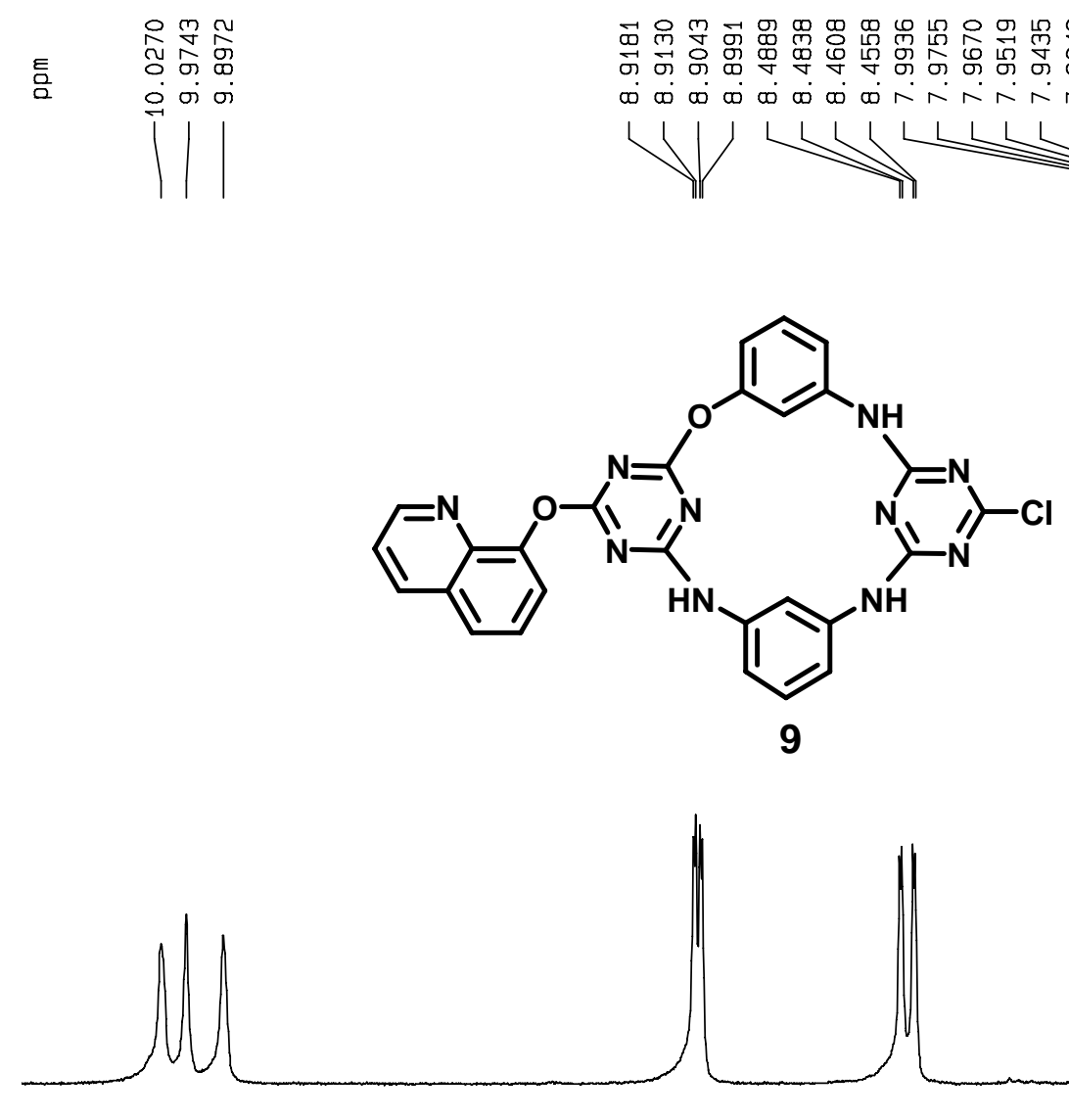

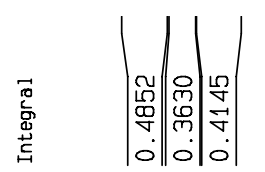

ppm 10.0
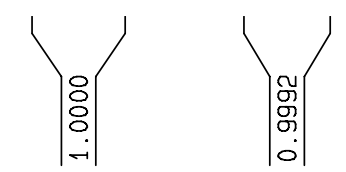

9.5

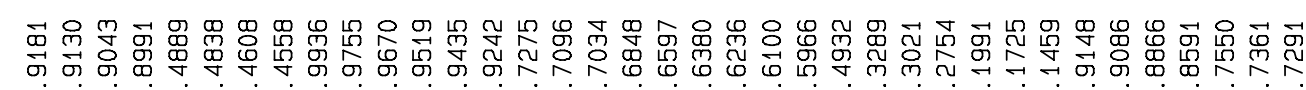
m
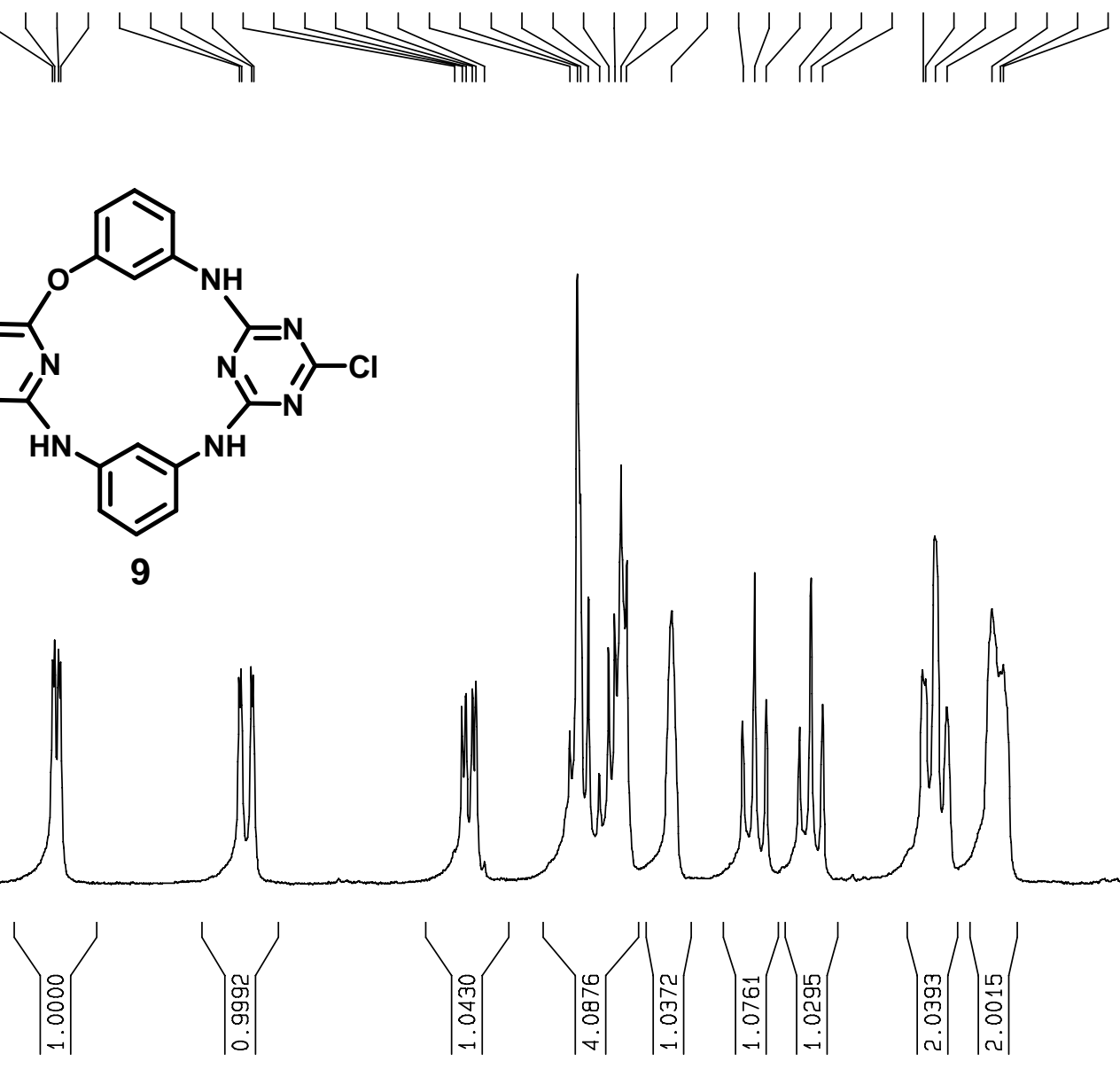

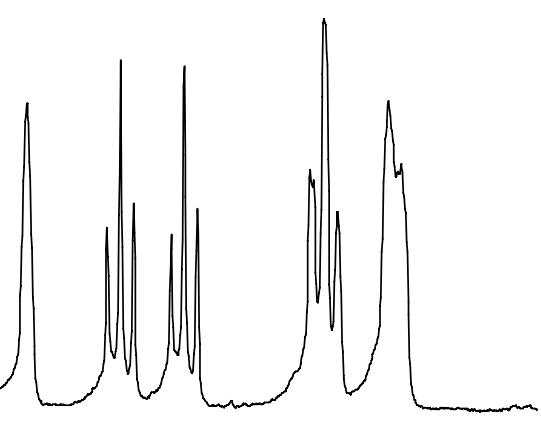

$\left|\begin{array}{l}\mid \\ \tilde{m} \\ 0 \\ 0 \\ \text { ن. }\end{array}\right|$
Current Data Parameters NAME yhb-1106-3n108q DROCNO

$=3-$ Acquisition Paraneters

Jate_$$
20041107
$$

INSTRUM 5.41
$-3 \times 300$ PROBHD $5 \mathrm{~mm}$ DUL $13 \mathrm{C} 300$ OULPROG $\begin{array}{lr}\text { TD } & \text { SOLVNT } \\ \text { SOLVET } & \text { DMSO }\end{array}$

NS

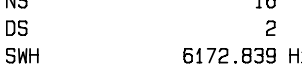

IDRES $\quad 0.094190 \mathrm{~Hz}$

AQ $\quad 5.3084660 \mathrm{sec}$

$\begin{array}{lr}\text { FG } & 322.5 \\ \text { JW } & 81.000 \text { usec }\end{array}$ $\begin{array}{r}6.00 \text { usec } \\ \hline\end{array}$ $2.00000000 \mathrm{sec}$

$=======$ CHANNEL $f 1$ =="==="= NUC1 $\quad 1 \mathrm{H}$ $\begin{array}{lr}P 1 & 9.30 \text { usec } \\ \text { PL1 } & -1.00 \mathrm{~dB}\end{array}$ SF01 $300.1318534 \mathrm{MHz}$

=2 - Processing parameters SI 32768 SF $\begin{array}{lc}\text { WDW } & \text { EM } \\ \text { SSB } & 0\end{array}$ $\begin{array}{cc}L B & 0.30 \mathrm{~Hz} \\ G B & 0 \\ { }^{\circ} & 1.00\end{array}$

10 NMR plot parameters

$\begin{array}{ll}\text { CX } & 22.00 \mathrm{~cm} \\ \mathrm{cY} & 25.00 \mathrm{~cm}\end{array}$ $\begin{array}{lc}\mathrm{CY} & 25.00 \mathrm{~cm} \\ =1 \mathrm{P} & 10.318 \mathrm{ppm}\end{array}$ $\begin{array}{ll}71 & 3096.82 \mathrm{~Hz}\end{array}$ $=2 \quad 1936.64 \mathrm{~Hz}$ $\begin{array}{ll} & 0.17571 \mathrm{ppm} / \mathrm{cm} \\ \text { HZCM } & 52.73531 \mathrm{~Hz} / \mathrm{cm}\end{array}$ 


\section{${ }^{13} \mathrm{C}$ NMR of $\mathbf{9}$}
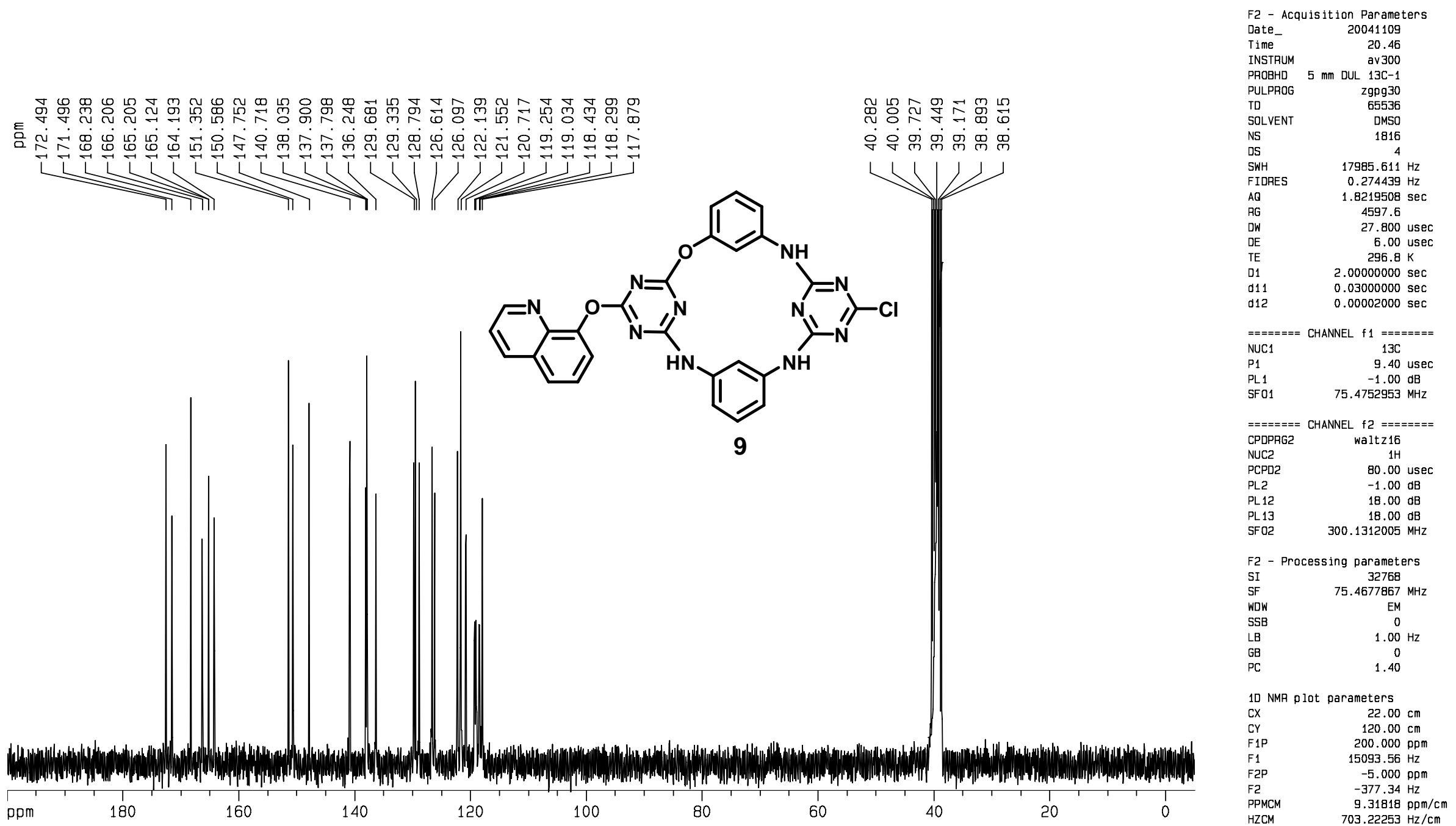

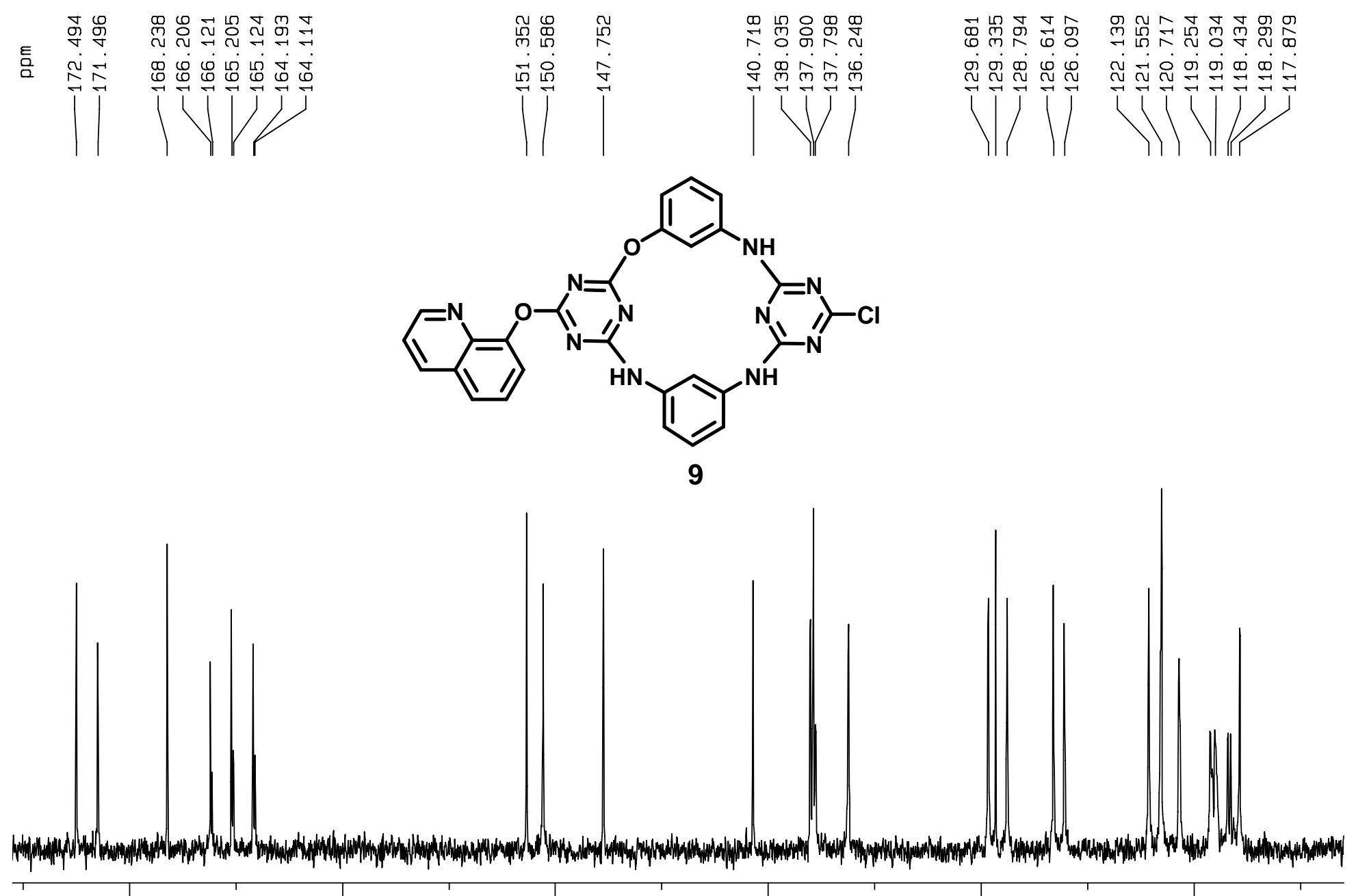

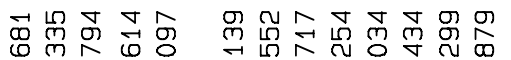

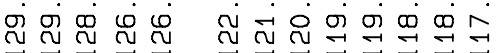
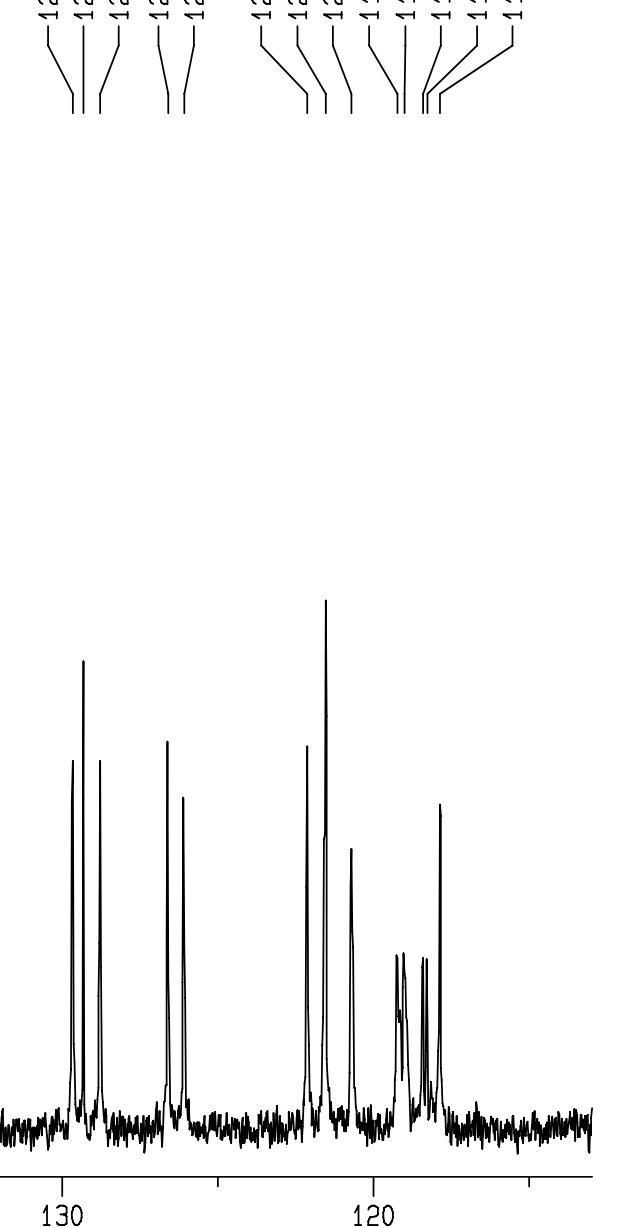

\begin{tabular}{|c|c|}
\hline \multicolumn{2}{|c|}{ F2 - Acquisition Parameters } \\
\hline Date_ & \\
\hline Time & 20.46 \\
\hline INSTRUM & av300 \\
\hline PROBHD & $5 \mathrm{~mm}$ DUL $13 \mathrm{C}-1$ \\
\hline PULPROG & zgpg30 \\
\hline TD & 65536 \\
\hline SOLVENT & DMSO \\
\hline NS & 1816 \\
\hline DS & 4 \\
\hline 5WH & $17985.611 \mathrm{~Hz}$ \\
\hline FIDRES & $0.274439 \mathrm{~Hz}$ \\
\hline AQ & $1.8219508 \mathrm{sec}$ \\
\hline RG & 4597.6 \\
\hline DW & 27.800 usec \\
\hline $\mathrm{DE}$ & 6.00 usec \\
\hline $\mathrm{TE}$ & $296.8 \mathrm{~K}$ \\
\hline 01 & $2.00000000 \mathrm{sec}$ \\
\hline d11 & $0.03000000 \mathrm{sec}$ \\
\hline $\mathrm{d} 12$ & $0.00002000 \mathrm{sec}$ \\
\hline$====== \pm$ & CHANNEL $f 1$ ======= \\
\hline NUC1 & $13 C$ \\
\hline$p_{1}$ & 9.40 usec \\
\hline PL1 & $-1.00 \mathrm{~dB}$ \\
\hline $5 F 01$ & $75.4752953 \mathrm{MHz}$ \\
\hline$==== \pm= \pm$ & CHANNEL f2 ======= \\
\hline CPDPRG2 & waltz16 \\
\hline NUC2 & \\
\hline PCPD2 & 80.00 usec \\
\hline PL2 & $-1.00 \mathrm{~dB}$ \\
\hline PL12 & $18.00 \mathrm{~dB}$ \\
\hline PL13 & $18.00 \mathrm{~dB}$ \\
\hline $\mathrm{SFO2}$ & $300.1312005 \mathrm{MHz}$ \\
\hline F2 - Pro & cessing parameters \\
\hline SI & 32768 \\
\hline $\mathrm{SF}$ & $75.4677867 \mathrm{MHZ}$ \\
\hline WOW & EM \\
\hline SSB & 0 \\
\hline LB & $1.00 \mathrm{~Hz}$ \\
\hline GB & 0 \\
\hline $\mathrm{PC}$ & 1.40 \\
\hline 10 NMR $p$ ? & lot parameters \\
\hline$c x$ & $22.00 \mathrm{~cm}$ \\
\hline $\mathrm{CY}$ & $80.00 \mathrm{~cm}$ \\
\hline F1P & $175.515 \mathrm{ppm}$ \\
\hline$F_{1}$ & $13245.76 \mathrm{~Hz}$ \\
\hline F2P & $112.998 \mathrm{ppm}$ \\
\hline F2 & $8527.71 \mathrm{~Hz}$ \\
\hline PPMCM & $2.84171 \mathrm{ppm} / \mathrm{cm}$ \\
\hline IZCM & $214.45718 \mathrm{~Hz} / \mathrm{cm}$ \\
\hline
\end{tabular}




\section{${ }^{1} \mathrm{H}$ NMR of 12a}

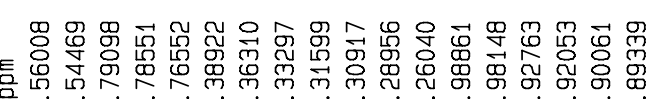

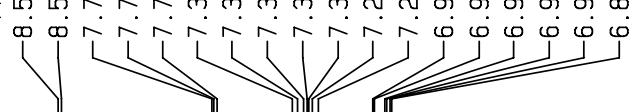

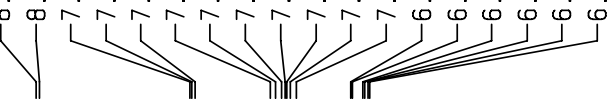

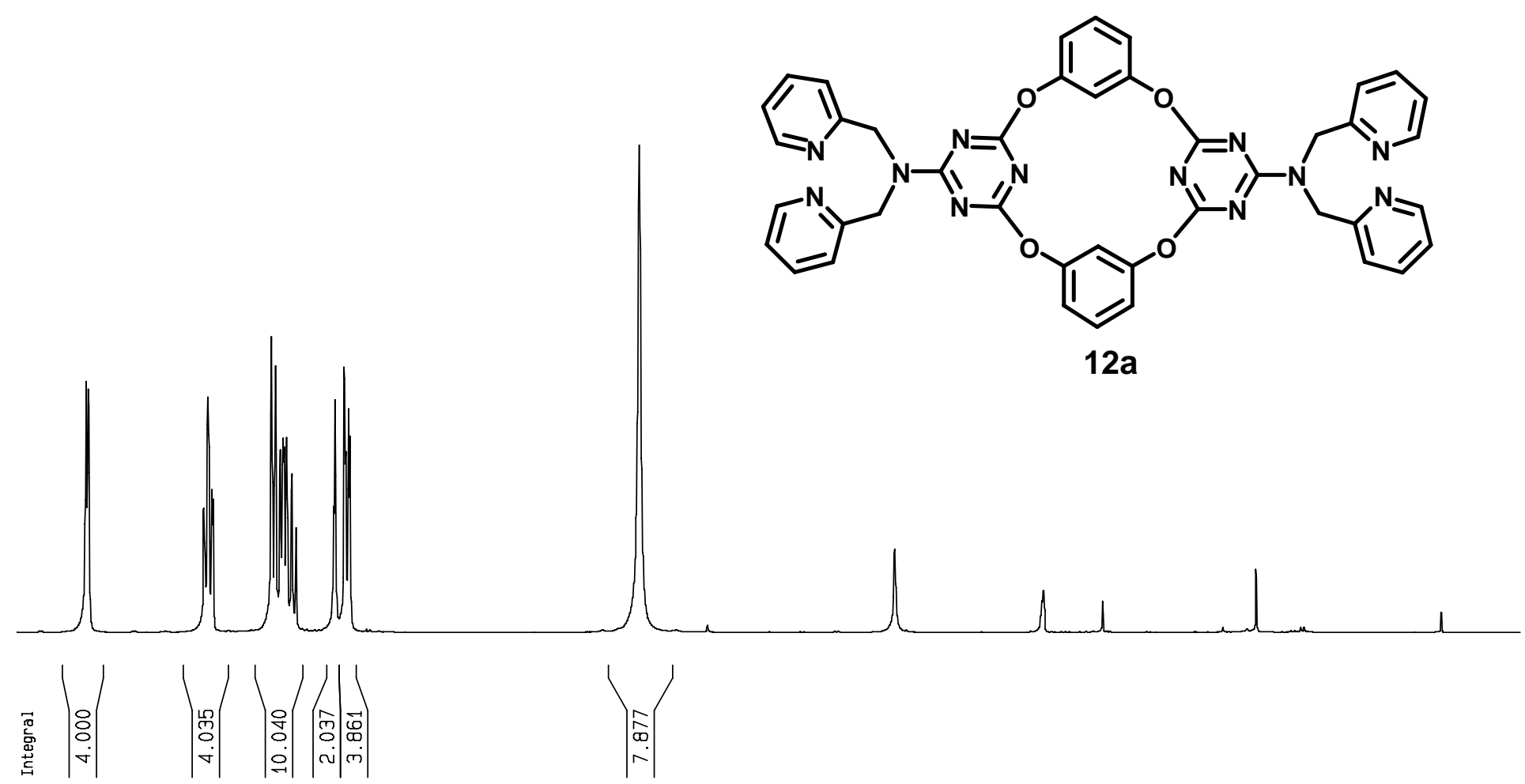

Current Data Parameters

NAME

PROCNO

F2 - Acquisition Parameters

Date_ 20031229

Time

5 av300

$\begin{array}{lr}\text { PULPROG } & 2930 \\ \text { TD } & 65536 \\ \text { SOLVENT } & \text { DMSO }\end{array}$

SOLVEN

SWH 2

SWH $\quad 6172.839 \mathrm{~Hz}$

AQ $\quad 5.3084660 \mathrm{sec}$

AG $\quad 80.6$

$\begin{array}{rr}\text { DW } & 81.000 \text { usec } \\ \text { DE } & 6.00 \text { usec }\end{array}$

TE $291.7 \mathrm{~K}$

$=======$ CHANNEL $f 1=====$

$=======$ CHANNEL
NUC1 $1=======$
PI

$\begin{array}{lll}P 1 & 9.30 \text { usec }\end{array}$

$\begin{array}{lr}-1.00 \mathrm{~dB} \\ \text { SF01 } & 300.1318534 \mathrm{MHz}\end{array}$

F2 - Processing parameters

SI 32768

WOW $\quad 300.1299962 \mathrm{MHz}$

WOW

LB
GB

PC

1.00

10 NMR plot parameters

$\begin{array}{lr}\text { CX } & 22.00 \mathrm{~cm}\end{array}$

$\begin{array}{lr}\text { EY } & 7.50 \mathrm{~cm} \\ =1 \mathrm{P} & 9.000 \mathrm{pPm}\end{array}$

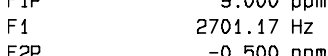

$\begin{array}{ll}\text { F2P } & -0.500 \mathrm{ppm} \\ F 2 & -150.07 \mathrm{~Hz}\end{array}$

PPMCM $\quad-150.07 \mathrm{~Hz}$

$\begin{array}{ll}0.43182 \mathrm{ppm} / \mathrm{cm} \\ \text { HZCM } & 129.60159 \mathrm{~Hz} / \mathrm{cm}\end{array}$ 


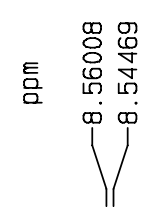
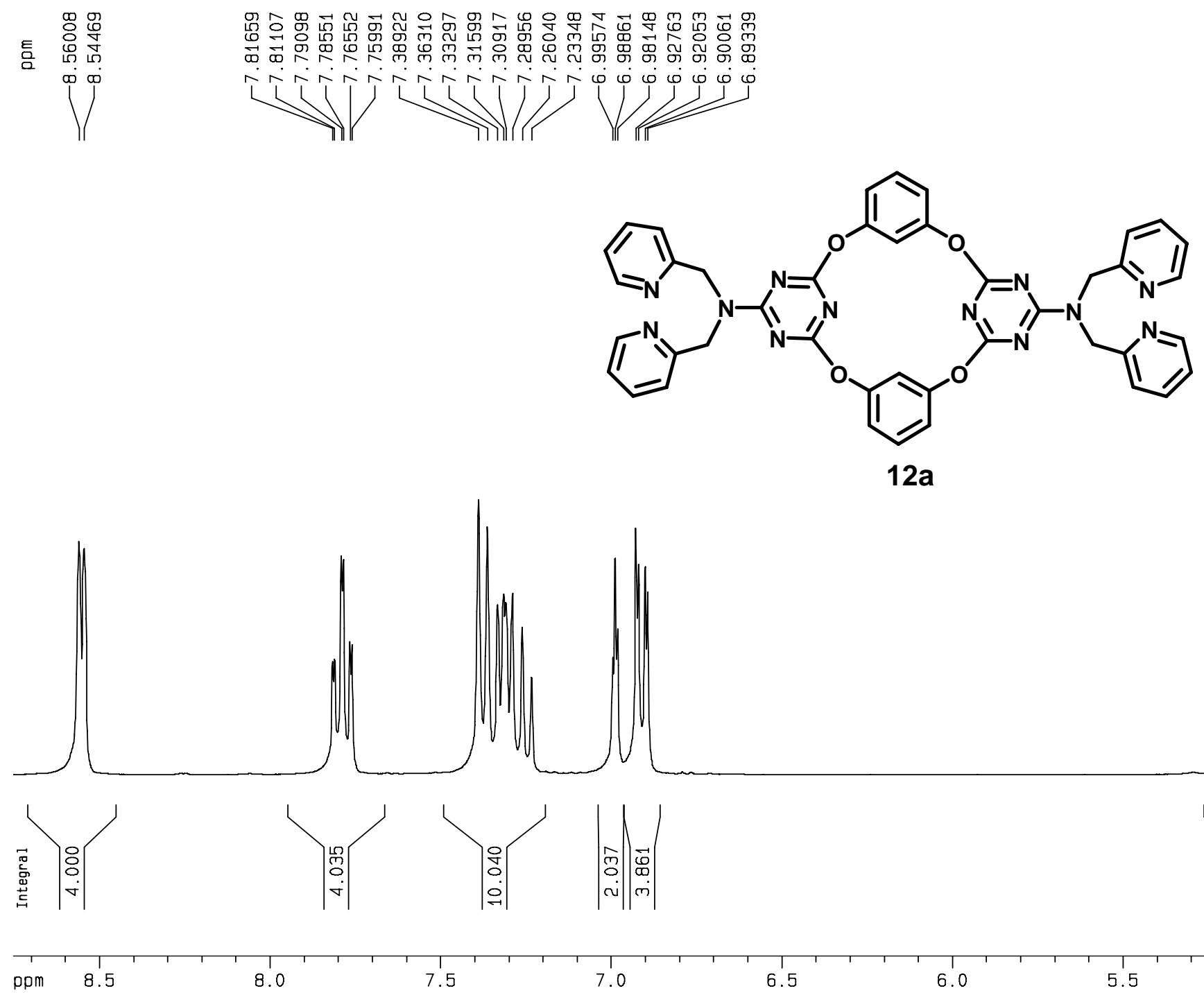

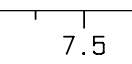

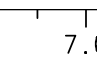

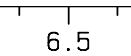

6.5

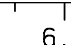

6.0

5.

5.5

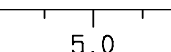

Current Data Parameters

NAME yhb031227a

PROCNO

F2 - Acquisition Parameter

Jate $\quad 20031229$

Time 0.18

INSTRUM av300

PULPROG 2930

SOLVENT DMSO

NS

$5 \mathrm{WH}$

=IDRES $\quad 6172.839 \mathrm{~Hz}$

AQ $\quad 0.094190 \mathrm{~Hz}$

$5.3084660 \mathrm{sec}$

JW

JE $\quad 6.00$ usec

$\begin{array}{ll}\text { TE } & 291.7 \mathrm{~K} \\ \text { J1 } & 2.00000000 \mathrm{sec}\end{array}$

$=======$ CHANNEL $f 1$ =======
NUC1

$\begin{array}{ll}\text { NOC1 } & 1 \mathrm{H} \\ \mathrm{P}_{1} & 9.30 \mathrm{useC}\end{array}$

PL1 $\quad-1.00 \mathrm{~dB}$

SF01 $300.1318534 \mathrm{MHz}$

F2 - Processing parameters

SI 32768

$\mathrm{SF} \quad 300.1299962 \mathrm{MHz}$

$\begin{array}{lr}\text { WDW } & \text { EM } \\ \text { SSB } & 0\end{array}$

LB $\quad 0.30 \mathrm{~Hz}$

$\begin{array}{lr}\mathrm{GB} & 0 \\ \mathrm{PC} & 1.00\end{array}$

10 NMA plot parameters

CX $\quad 22.00 \mathrm{~cm}$

$\begin{array}{ll}C Y & 7.50 \mathrm{~cm} \\ =1 \mathrm{P} & 0.753 \mathrm{com}\end{array}$

$\begin{array}{ll}F 1 & 8.753 \mathrm{ppm} \\ = & 2627.04 \mathrm{~Hz}\end{array}$

$\begin{array}{ll}72 \mathrm{P} & 4.799 \mathrm{ppm} \\ =2 & 140.24 \mathrm{~Hz}\end{array}$

PPMCM $\quad 0.17974 \mathrm{pDm} / \mathrm{cm}$

$\begin{array}{ll}\text { HZCM } & 53.94537 \mathrm{~Hz} / \mathrm{cm}\end{array}$ 
${ }^{13} \mathrm{C}$ NMR of 12a

हl|
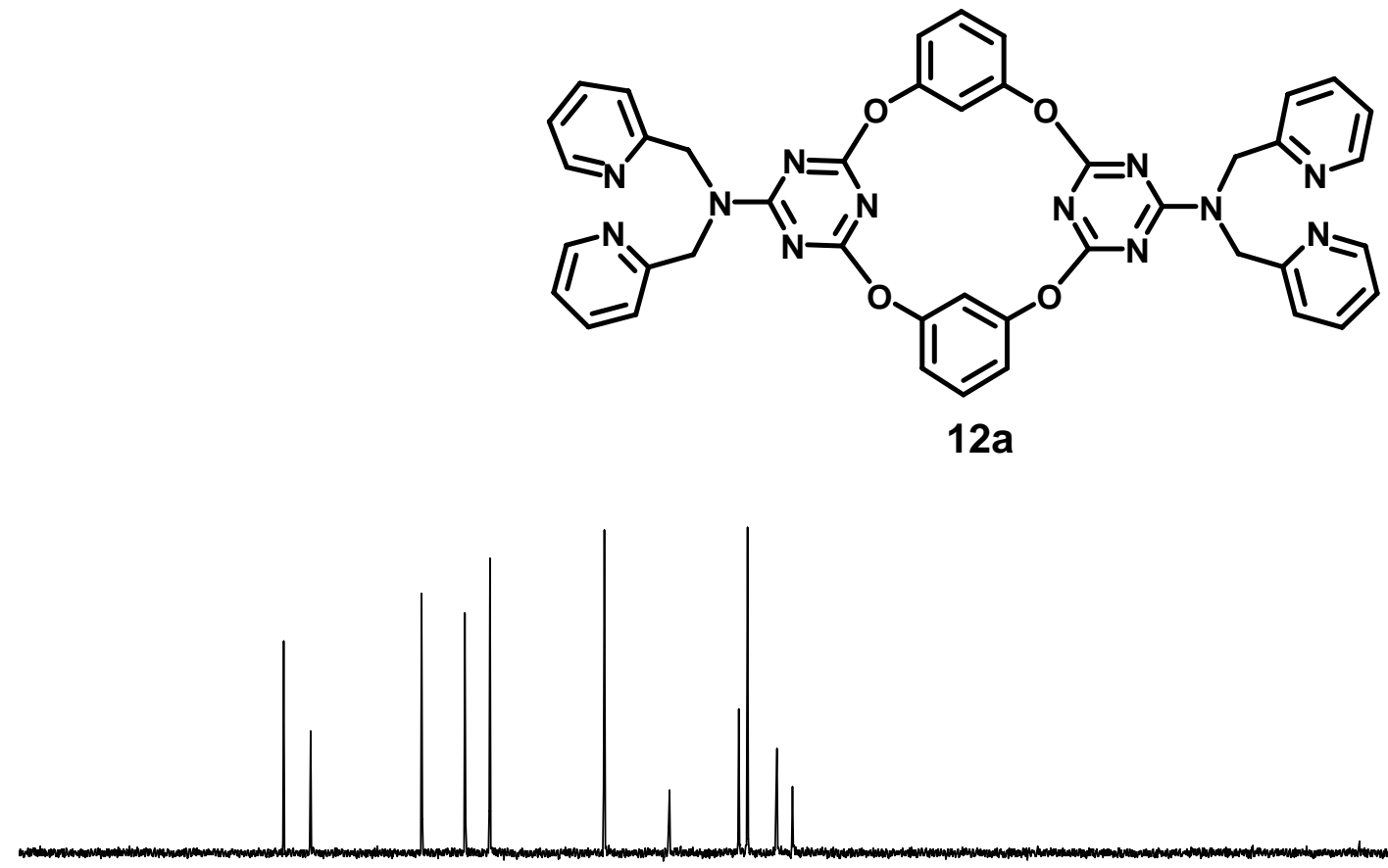

ppm

180
160
120
140

100

80

60

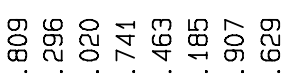

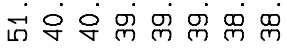

14

40

20

20

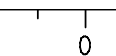

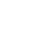

F2 - Acquisition Parameters

Jate_

Time

0.29
ThSTRUM

PULPROG

$\begin{array}{lr}\text { TD } & 65536 \\ \text { SOLVENT } & \text { DMSO }\end{array}$

289
NS

SWH $\quad 17985.611 \mathrm{~Hz}$

$\begin{array}{ll}\text { FIDRES } & 0.274439 \mathrm{~Hz} \\ \mathrm{AO} & 1.8219508 \mathrm{sec}\end{array}$

$\begin{array}{ll}\text { AG } & 1448.2 \\ \text { JW } & 27.800 \text { use }\end{array}$

JE $\quad 6.00$ usec

$292.2 \mathrm{~K}$

$2.00000000 \mathrm{sec}$

0.00002000 sec

J12 0.030000000

$=======$ CHANNEL $f 1$ $1=======$
NUC1 $13 \mathrm{C}$

$\begin{array}{lr}\text { P1 } & 9.40 \mathrm{usec} \\ \text { PL1 } & -1.00 \mathrm{~dB}\end{array}$

$\begin{array}{lr}\text { PL1 } & -1.00 \mathrm{~dB} \\ \text { SF } 01 & 75.4752953 \mathrm{MHz}\end{array}$

$=======$ CHANNEL f2 =="==="=

CPDPRG2 waltz16

$\begin{array}{lc}\text { NUC2 } & 1 \mathrm{H} \\ \text { PCPD2 } & 80.00 \mathrm{usec}\end{array}$

PL2 $\quad-1.00 \mathrm{dg}$

PL13 $18.00 \mathrm{~dB}$

$300.1312005 \mathrm{MHZ}$

F2 - Processing parameters

$\begin{array}{ll} & \\ \text { SI } & 32768 \\ \text { SF } & 75.4677867 \mathrm{MHz}\end{array}$

WDW $\quad$ EM

$\begin{array}{lc}S S B & 0 \\ L B & 1.00 \mathrm{~Hz} \\ G B & 0 \\ P C & 1.40\end{array}$

NMP plot parameters

cX $\quad 22.00 \mathrm{~cm}$

$\begin{array}{ll}\mathrm{CY} & 7.50 \mathrm{~cm} \\ =1 \mathrm{P} & 200.000 \mathrm{ppm}\end{array}$

$\begin{array}{ll}=1 & 15093.56 \mathrm{~Hz} \\ =2 \mathrm{P} & -5.000 \mathrm{ppm}\end{array}$

=2 $\quad-377.34 \mathrm{~Hz}$

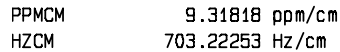




\section{${ }^{1} \mathrm{H}$ NMR of $\mathbf{1 2 b}$}

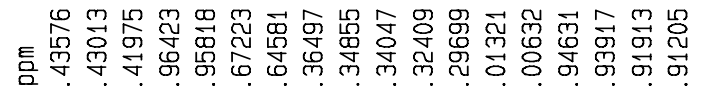

ᄂ

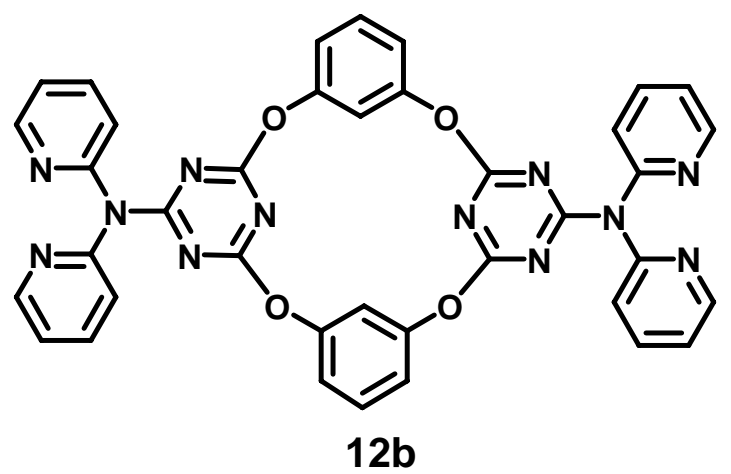

Lillu

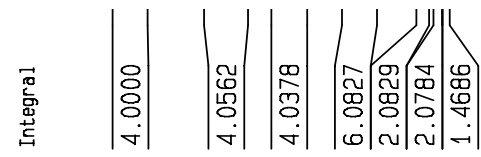

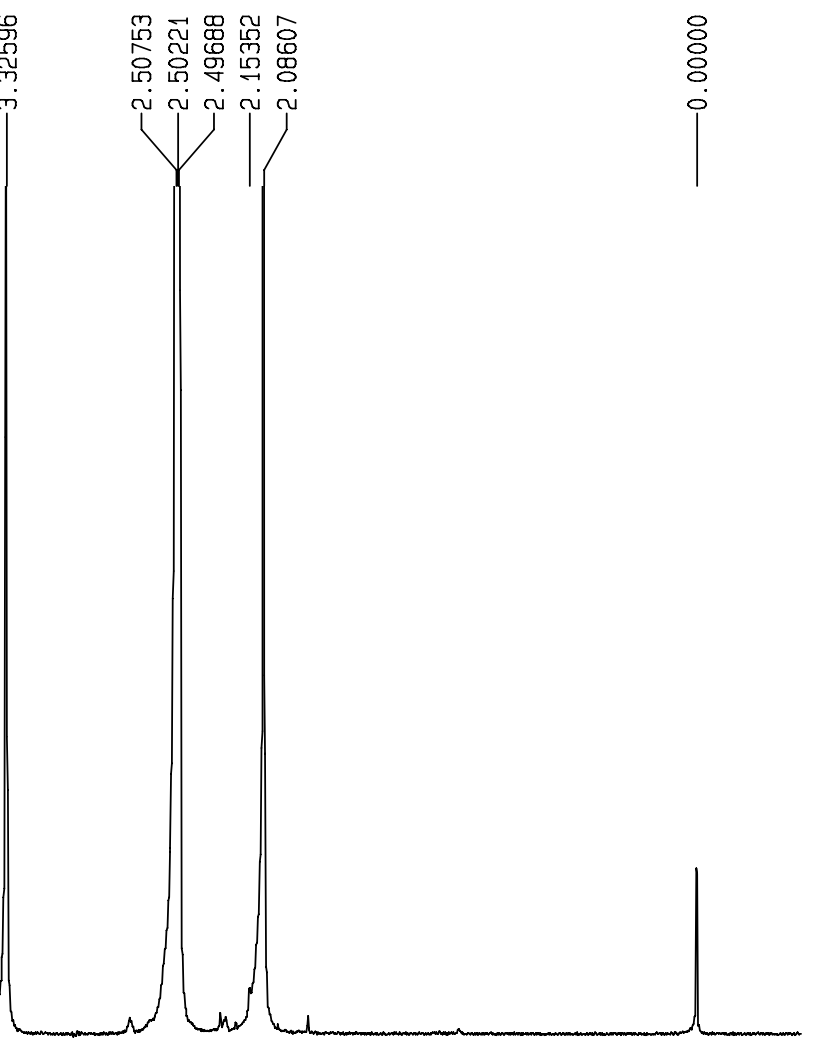

ppm

8

7

6

5

4

3

2

0 

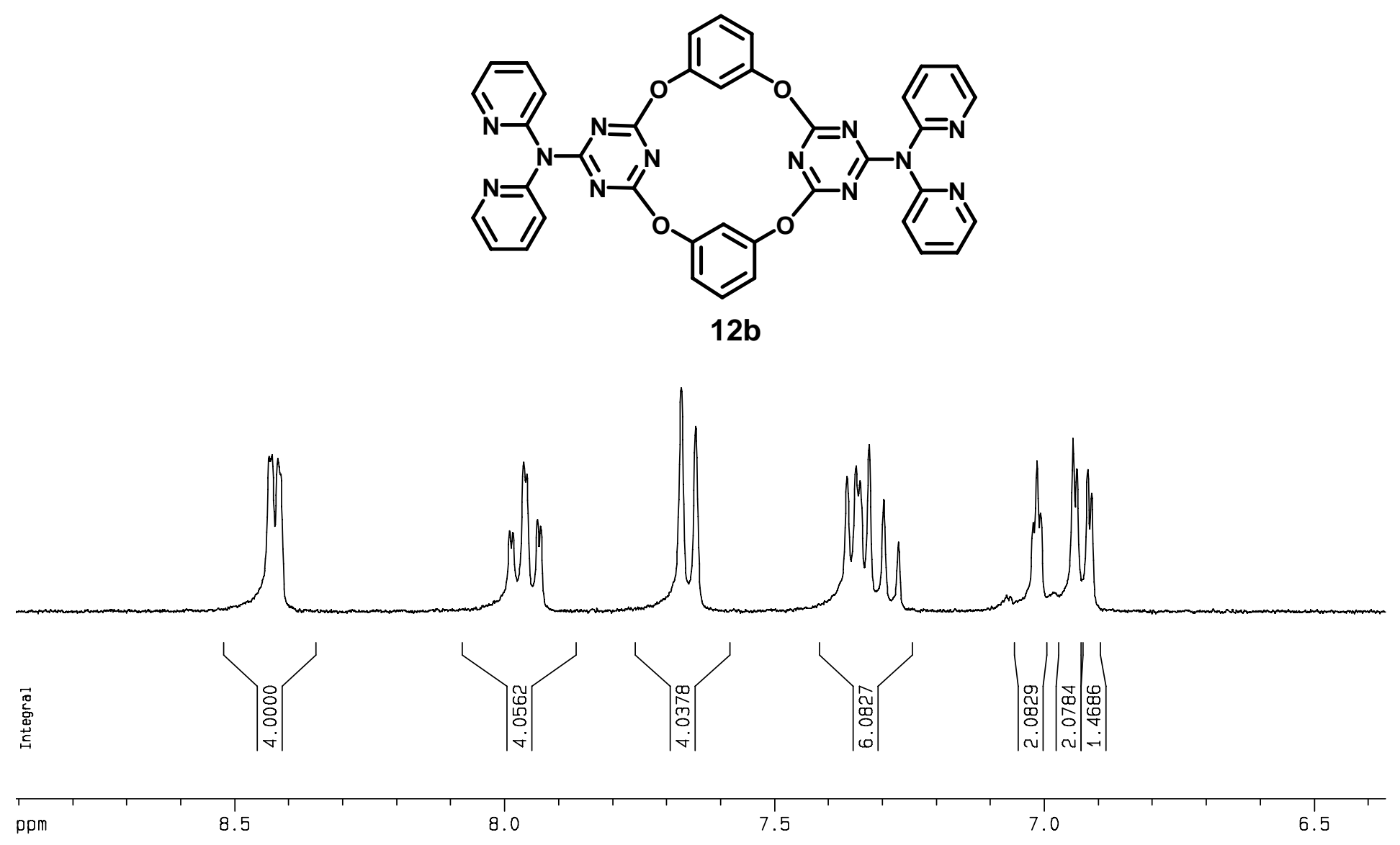

Current Data Parameters

F2 - Acquisition Parameters

Date

Time-

PULPROG 5 MI DUL 13

TD

SOLVENT

NS
DS
SWH

2
SWH
$6172.839 \mathrm{~Hz}$

FIDRES $\quad 0.094190 \mathrm{~Hz}$

AQ $\quad 5.3084660 \mathrm{sec}$

$\begin{array}{ll}\text { RG } & 406.4 \\ \text { DW } & 81.000 \text { use }\end{array}$

DE $\quad 6.00$ usec

$\begin{array}{lc}\text { TE } & 297.0 \mathrm{~K} \\ \text { D1 } & 2.00000000 \mathrm{sec}\end{array}$

$======$ CHANNEL $f 1$ 1 $======$
NUC1

NUC1 $1 \mathrm{H}$

$\begin{array}{lr}P 1 & 9.30 \text { usec } \\ P L 1 & -1.00 \mathrm{~dB}\end{array}$

F2 - Processing parameters

SI 32768

SF $\quad 300.1300007 \mathrm{MHZ}$

WOW

$\begin{array}{lr}5 S B & 0 \\ L B & 0.30 \mathrm{~Hz}\end{array}$

$\begin{array}{lr}G B & 0 \\ P C & 1.00\end{array}$

10 NMF plot parameters

cX $\quad 22.00 \mathrm{~cm}$

$\begin{array}{lr}\text { CY } & 100.00 \mathrm{~cm}\end{array}$

$\begin{array}{ll}\mathrm{F}_{1} & 8.906 \mathrm{ppm} \\ \mathrm{F} & 2672.85 \mathrm{~Hz}\end{array}$

$\begin{array}{lr}\text { F2P } & 6.368 \mathrm{ppm} \\ F 2 & 1911.25 \mathrm{~Hz}\end{array}$

$\begin{array}{ll}\text { F2 } & 1911.26 \mathrm{~Hz} \\ \text { PPMCM } & 0.11534 \mathrm{pDm} / \mathrm{c}\end{array}$

$\begin{array}{ll} & 0.11534 \mathrm{pmm} / \mathrm{cm} \\ \text { HZCM } & 34.61765 \mathrm{~Hz} / \mathrm{cm}\end{array}$ 
${ }^{13} \mathrm{C}$ NMR of $\mathbf{1 2 b}$
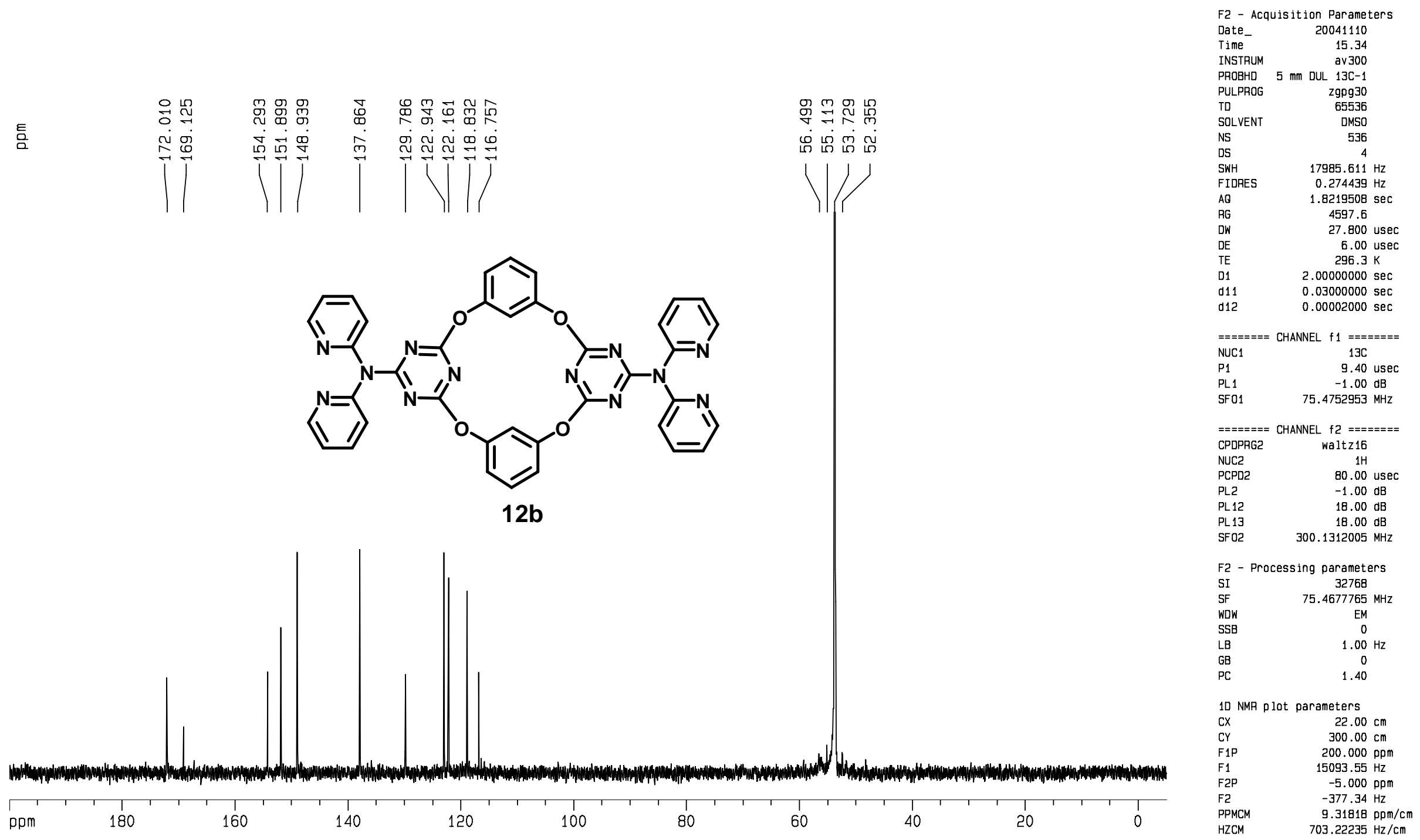


\section{${ }^{1} \mathrm{H}$ NMR of 12c}

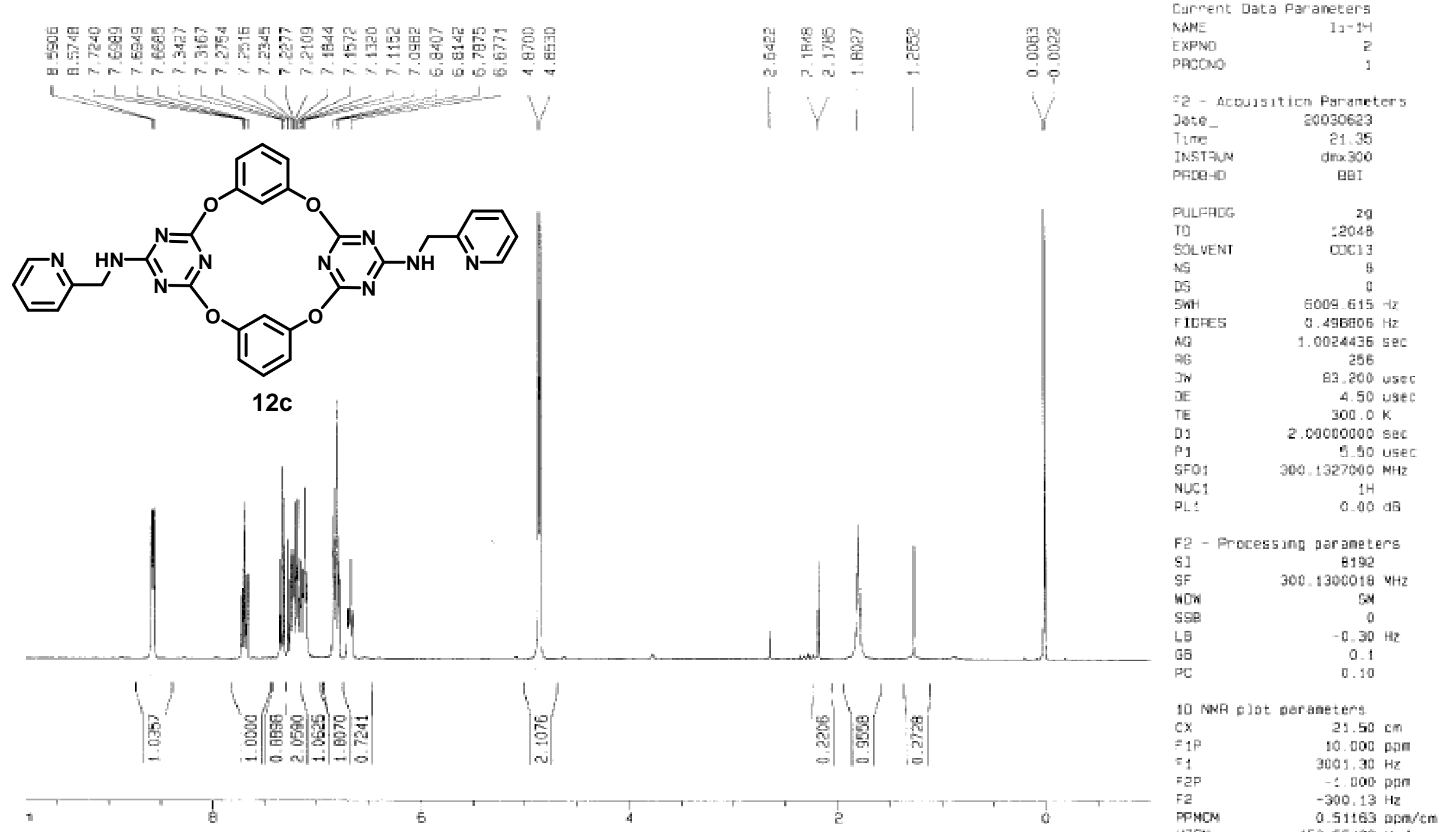


${ }^{13} \mathrm{C}$ NMR of 12c

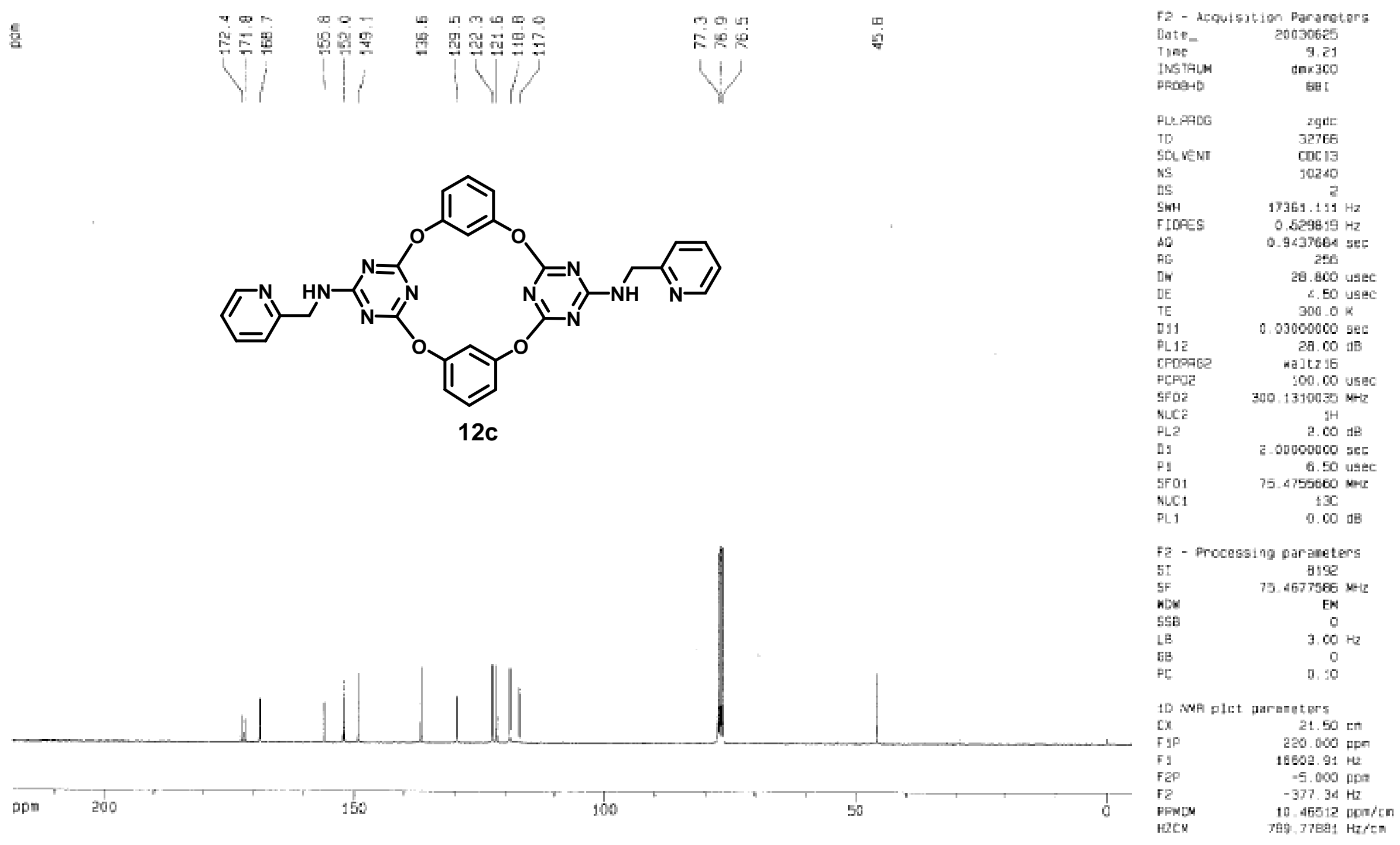


${ }^{1} \mathrm{H}$ NMR of 12d

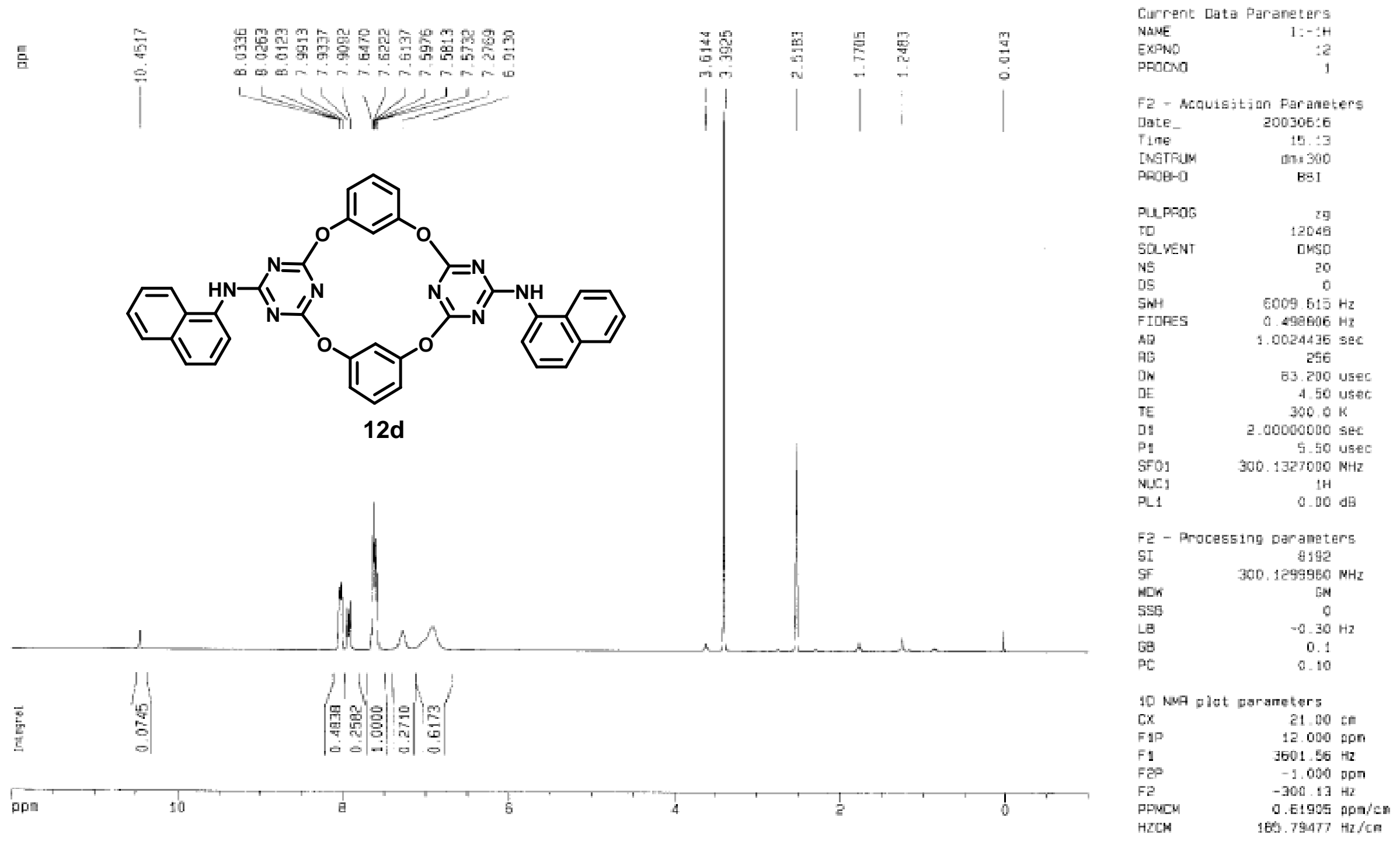


${ }^{13} \mathrm{C}$ NMR of 12d<smiles>c1cc2cc(c1)Oc1nc(Nc3cccc4ccccc34)nc(n1)Oc1cccc(c1)Oc1nc(Nc3cccc4ccccc34)nc(n1)O2</smiles> 
${ }^{1} \mathrm{H}$ NMR of 12e

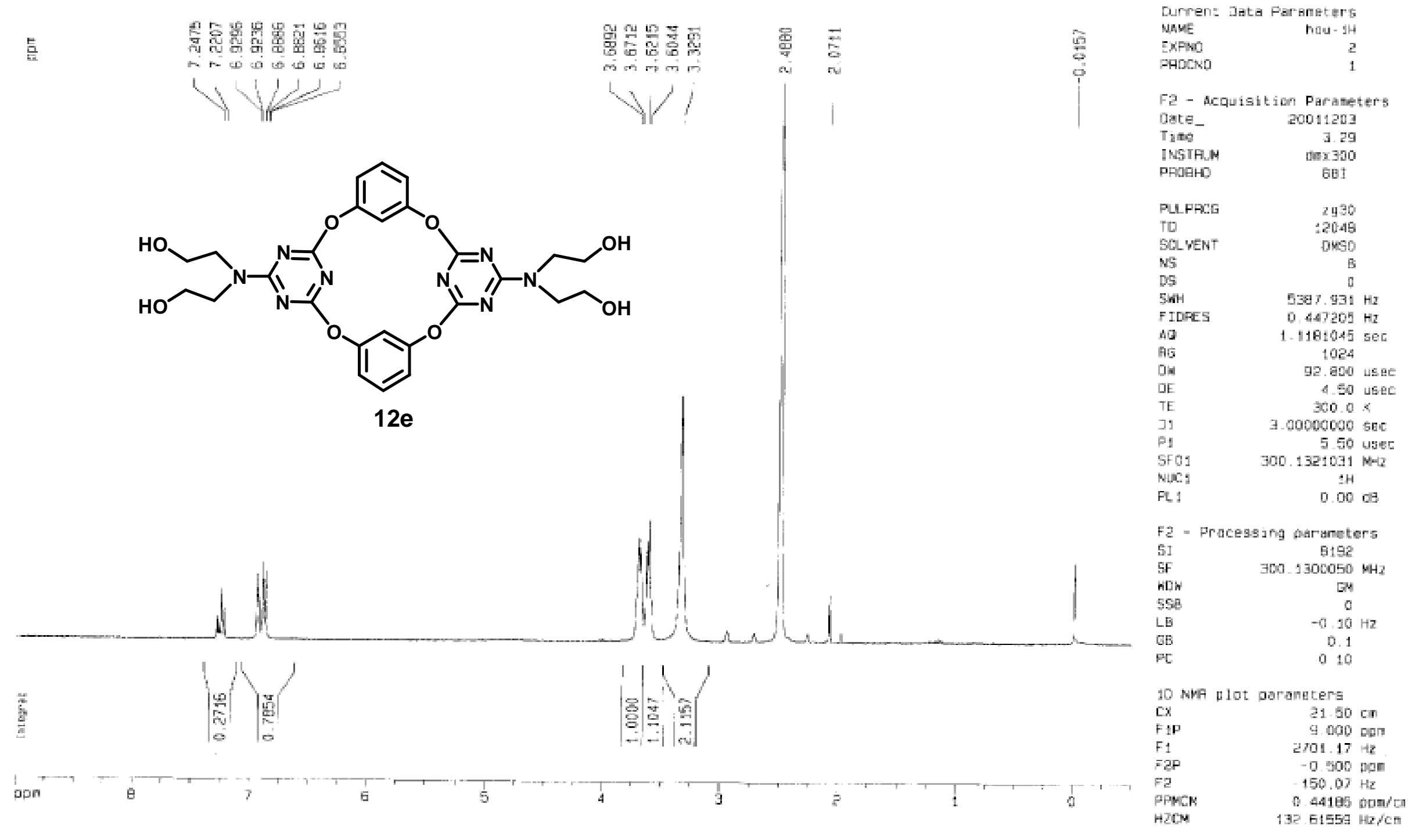


${ }^{13} \mathrm{C}$ NMR of 12e

|

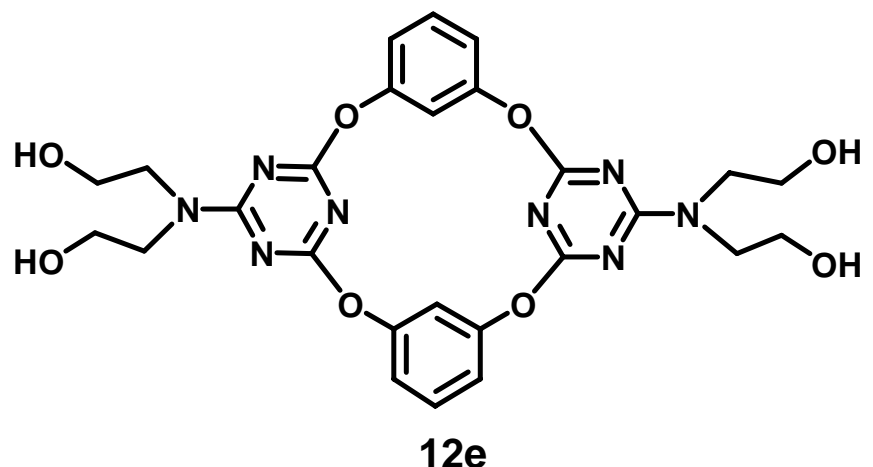

$\mid$ a

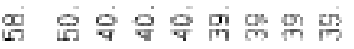

11111

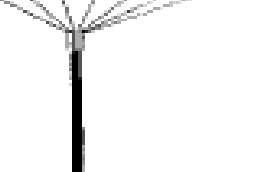

$1_{160}^{1}$

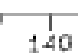

140

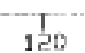

100

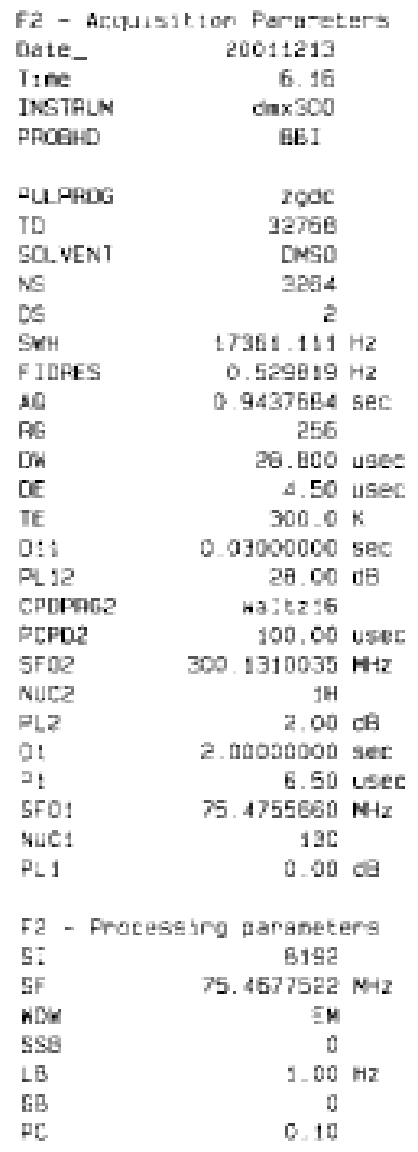

10 NMP a gor poraneter

CX N

$\begin{array}{ll}2 x & 21.50 \mathrm{~cm}\end{array}$

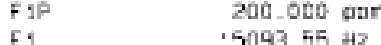

5000 .

$-377.34 \mathrm{~Hz}$

9. $534 \mathrm{BB}$ ond/C

3: $532 \mathrm{BB}$ pando 


\section{${ }^{1} \mathrm{H}$ NMR of 13a}

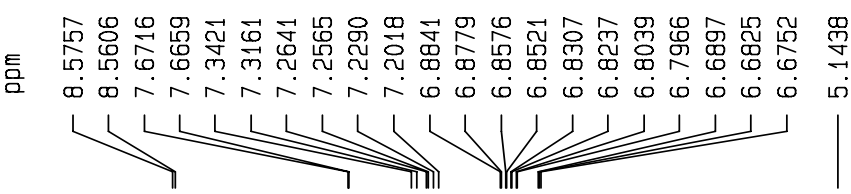

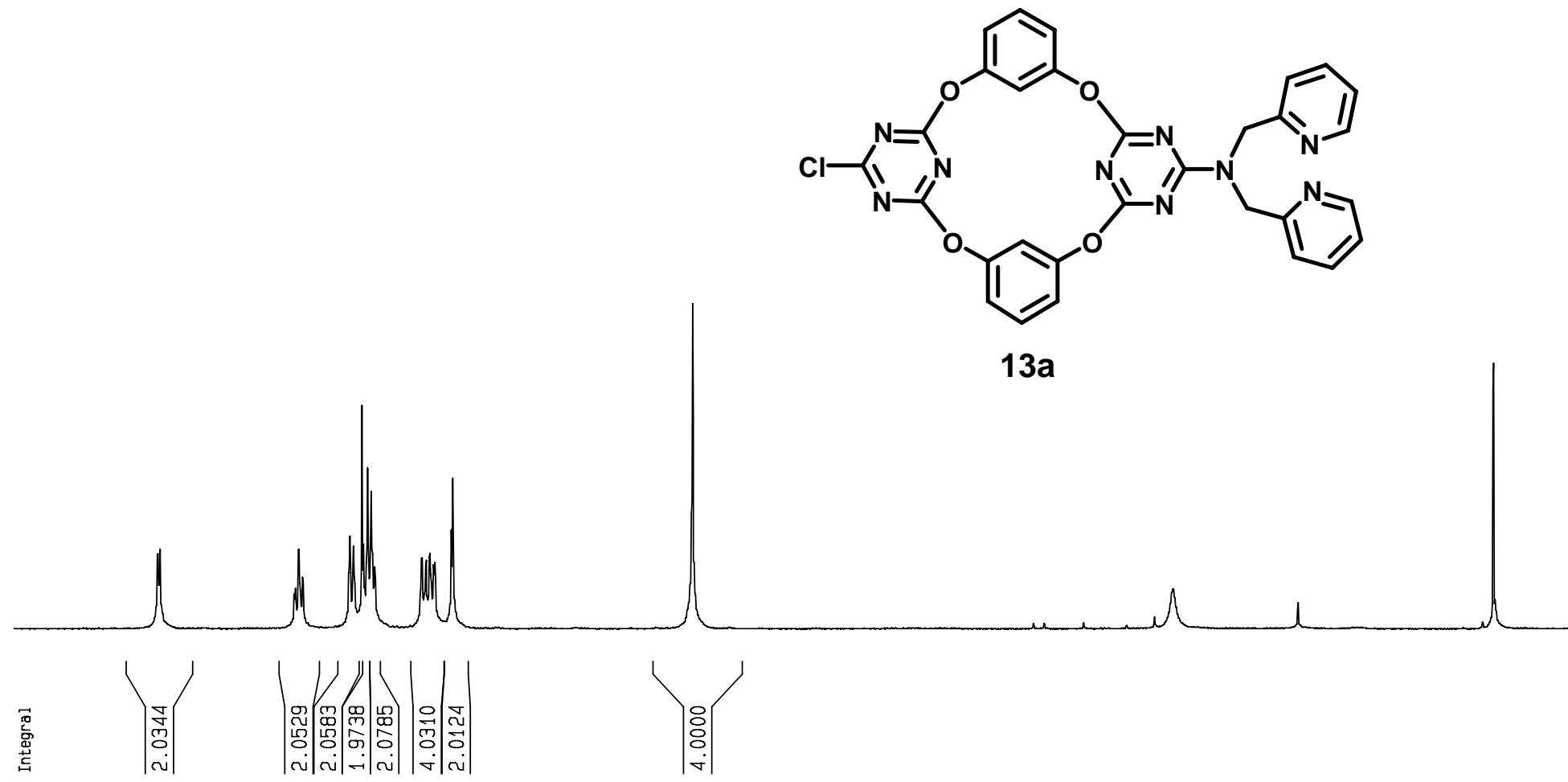

Current Data Parameters

NAME
EXPNO

PROCNO

F2 - Acquisition Parameters

Date

INSTRUM

11.03

$\begin{array}{rr}\text { PULPROG } & 2930 \\ \text { TD } & 65536\end{array}$

$\begin{array}{ll}\text { TD } & 65536 \\ \text { SOLVENT } & \text { CDC13 }\end{array}$

$\begin{array}{lr}\text { NS } & 16 \\ \text { DS } & 2 \\ \text { SWH } & 6172.839 \mathrm{~Hz}\end{array}$

FIDRES $\quad 0.094190 \mathrm{~Hz}$

$A Q \quad 5.3084660 \mathrm{sec}$

$\begin{array}{lr}\text { AG } & 512 \\ \text { DW } & 81.000 \text { usec }\end{array}$

TE $\quad 6.00$ us

$=======$ CHANNEL $f 1=======$
NUC1 $1 \mathrm{H}$

519.30 use

$300.1310534 \mathrm{dBz}$

F2 - Processing parameters

SI 32768

SF $\quad 300.1300048 \mathrm{MHz}$

$\begin{array}{lr}\text { WDW } & \text { EM } \\ 5 S B & 0\end{array}$

$\begin{array}{ll}\angle B & 0.35 \mathrm{~Hz} \\ G B & 0 \\ P C & 1.00\end{array}$

10 NMP plot parameters

$\begin{array}{lr}C X & 22.00 \mathrm{~cm} \\ \mathrm{CY} & 5.00 \mathrm{~cm}\end{array}$

$9.500 \mathrm{pp}$
$-1951.24 \mathrm{~Hz}$

$\begin{array}{ll}F 1 & 2851.24 \mathrm{~Hz} \\ \text { F2P } & -0.500 \mathrm{PP}\end{array}$

$-150.06 \mathrm{~Hz}$
$0.45455 \mathrm{ppm} / \mathrm{cm}$

$0.45455 \mathrm{ppm} / \mathrm{cm}$
$136.42273 \mathrm{~Hz} / \mathrm{cm}$

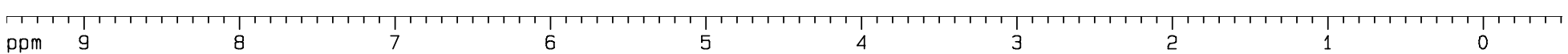


E

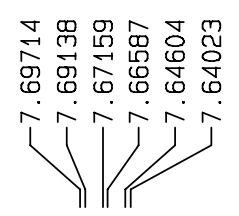

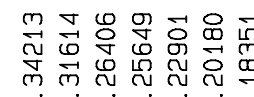

TNTITS
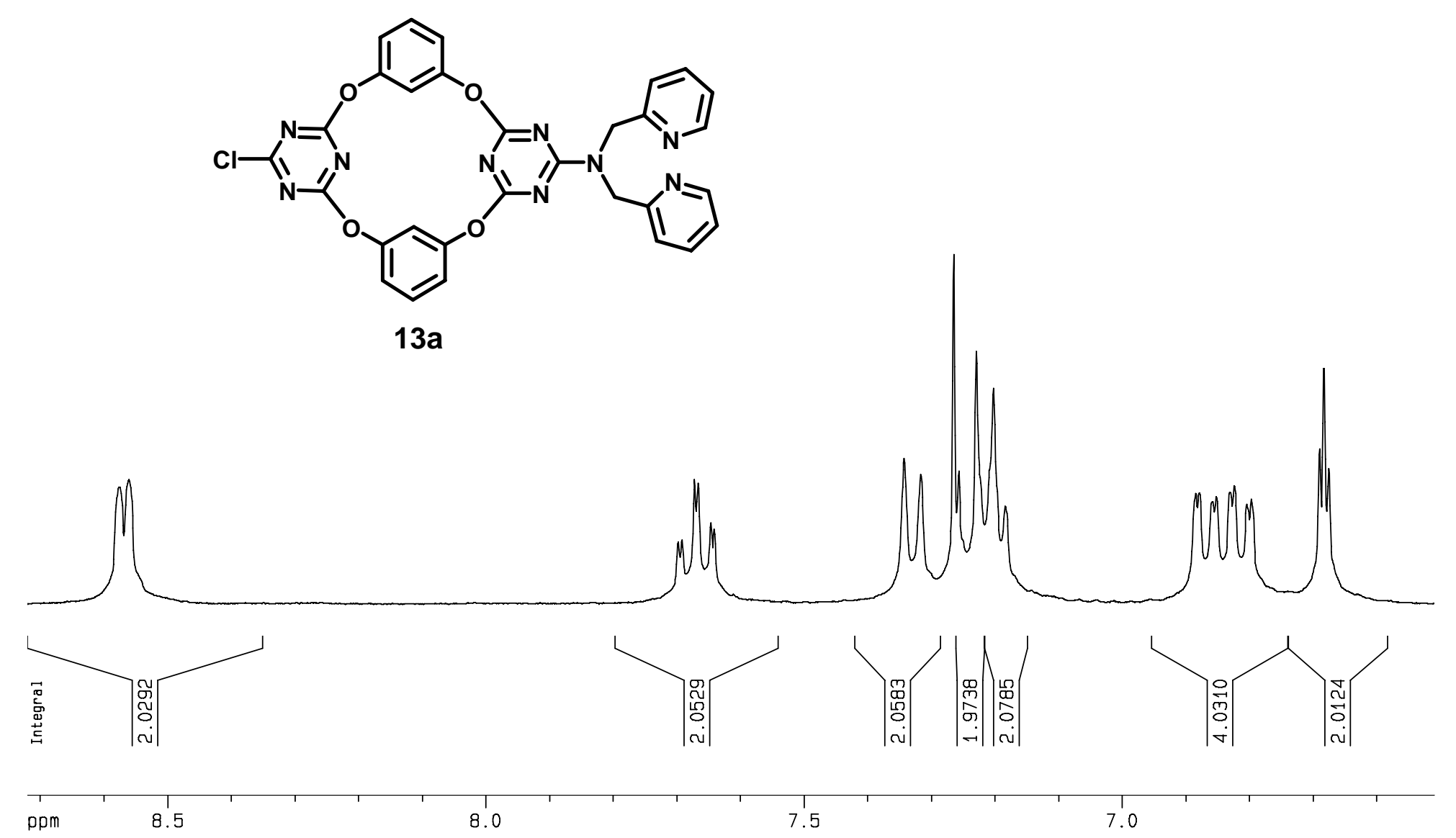

ppm

8.5

8.0

7.5

7.0

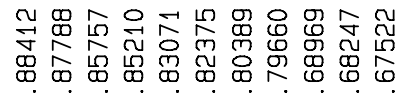

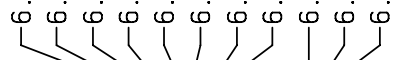

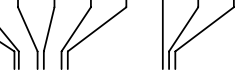

Current Data Parameters

NAME Wqq3-79a

EXPNO
PROCNO

20

F2 - Acquisition Parameters

Date__ 20070208

Time $\quad 11.03$

$\begin{array}{ll}\text { INSTRUM } & \text { aV300 } \\ \text { PROBHD } 5 \mathrm{~mm} \text { DUL } 13 C-1\end{array}$

PULPROG $5 \mathrm{~mm}$ DUL $13 \mathrm{C}-1$

$\begin{array}{lr}\text { TD } & 65536 \\ \text { SOLVENT } & \text { CDC13 }\end{array}$

NS

DS

5WH $\quad 6172.839 \mathrm{~Hz}$

FIDRES $\quad 0.094190 \mathrm{~Hz}$

AQ $\quad 5.3084660 \mathrm{sec}$

$\begin{array}{lr}\text { AG } & 512 \\ \text { DW } & 81.000 \text { usec }\end{array}$

DE $\quad 6.00$ usec

D1 $2.00000000 \mathrm{~K}$

$======$ CHANNEL $f 1$
NUC1

$\begin{array}{lr}\text { NOC1 } & 1 \mathrm{H} \\ \text { P1 } & 9.30 \text { usec } \\ \text { PL1 } & -1.00 \mathrm{~dB}\end{array}$

SF01 $300.1318534 \mathrm{MHz}$

F2 - Processing parameters

SI 32768

SF $\quad 300.1300048 \mathrm{MHZ}$

$\begin{array}{lr}\text { WOW } & \text { EM } \\ \text { SSB } & 0\end{array}$

$\begin{array}{cc}L B & 0.35 \mathrm{~Hz} \\ G B & 0\end{array}$

10 NMR plot parameters
CX $22.00 \mathrm{~cm}$

$\begin{array}{lr}\text { CX } & 22.00 \mathrm{~cm} \\ \mathrm{CY} & 8.00 \mathrm{~cm} \\ \mathrm{CHP} & 8.720 \mathrm{cpm}\end{array}$

$\begin{array}{lr}F_{10} & 8.720 \mathrm{ppm} \\ F_{1} & 2617.23 \mathrm{~Hz}\end{array}$

$\begin{array}{lr}\text { F२P } & 6.720 \mathrm{ppm} \\ \mathrm{F} & 6.510 \mathrm{ppm}\end{array}$

$\begin{array}{ll}\text { F2 } & 1953.78 \mathrm{~Hz} \\ \text { PPMCM } & 0.1008 \mathrm{pm}\end{array}$

$\begin{array}{lc}\text { PPMCM } & 0.10048 \mathrm{ppm} / \mathrm{cm} \\ \text { HZCM } & 30.15676 \mathrm{~Hz} / \mathrm{cm}\end{array}$ 
${ }^{13} \mathrm{C}$ NMR of 13a
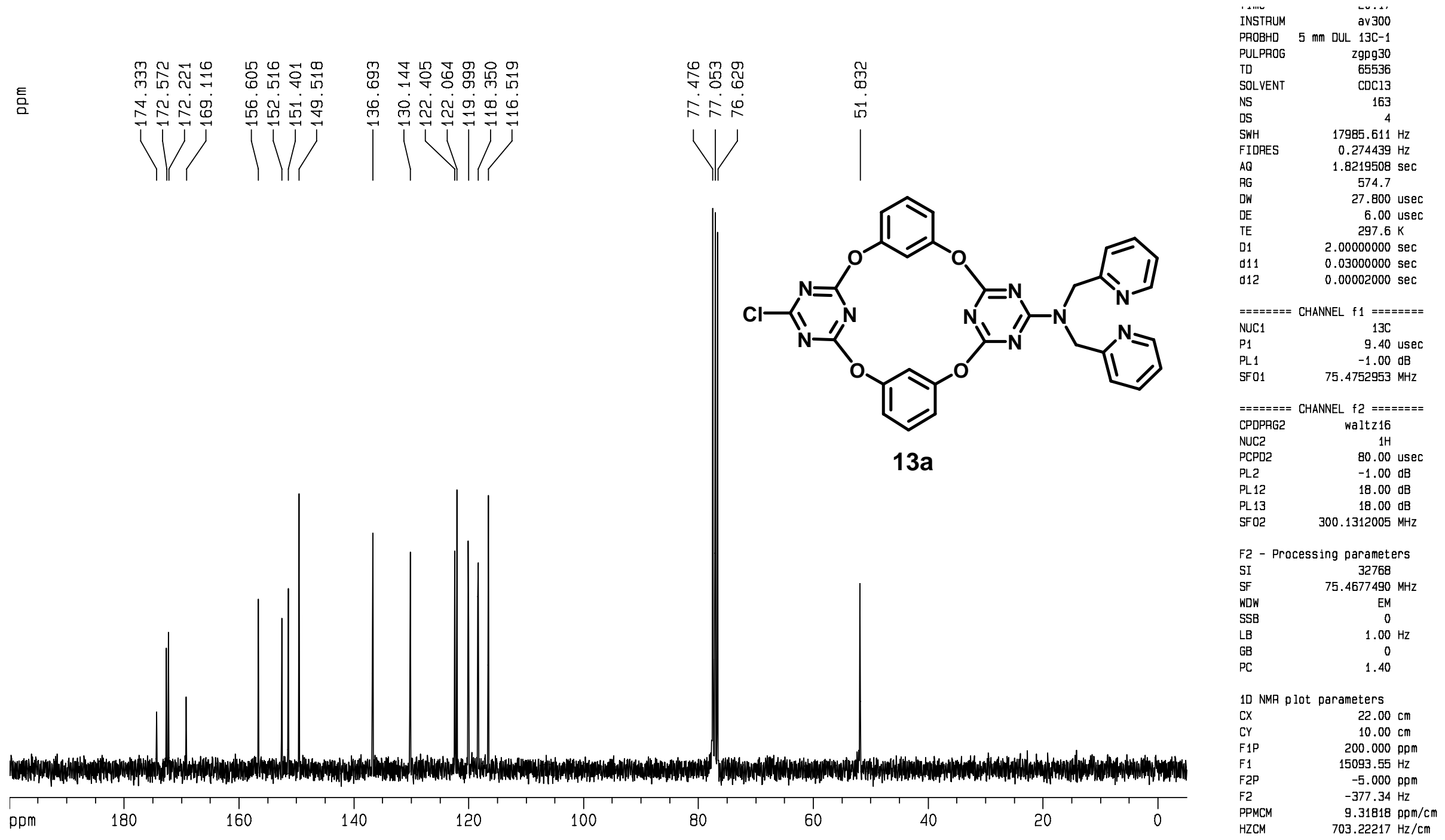
${ }^{1} \mathrm{H}$ NMR of $\mathbf{1 3 b}$

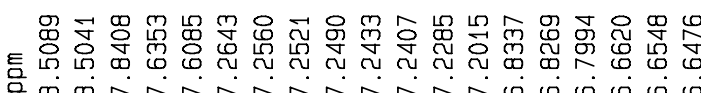

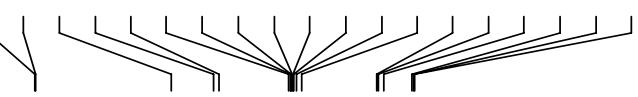

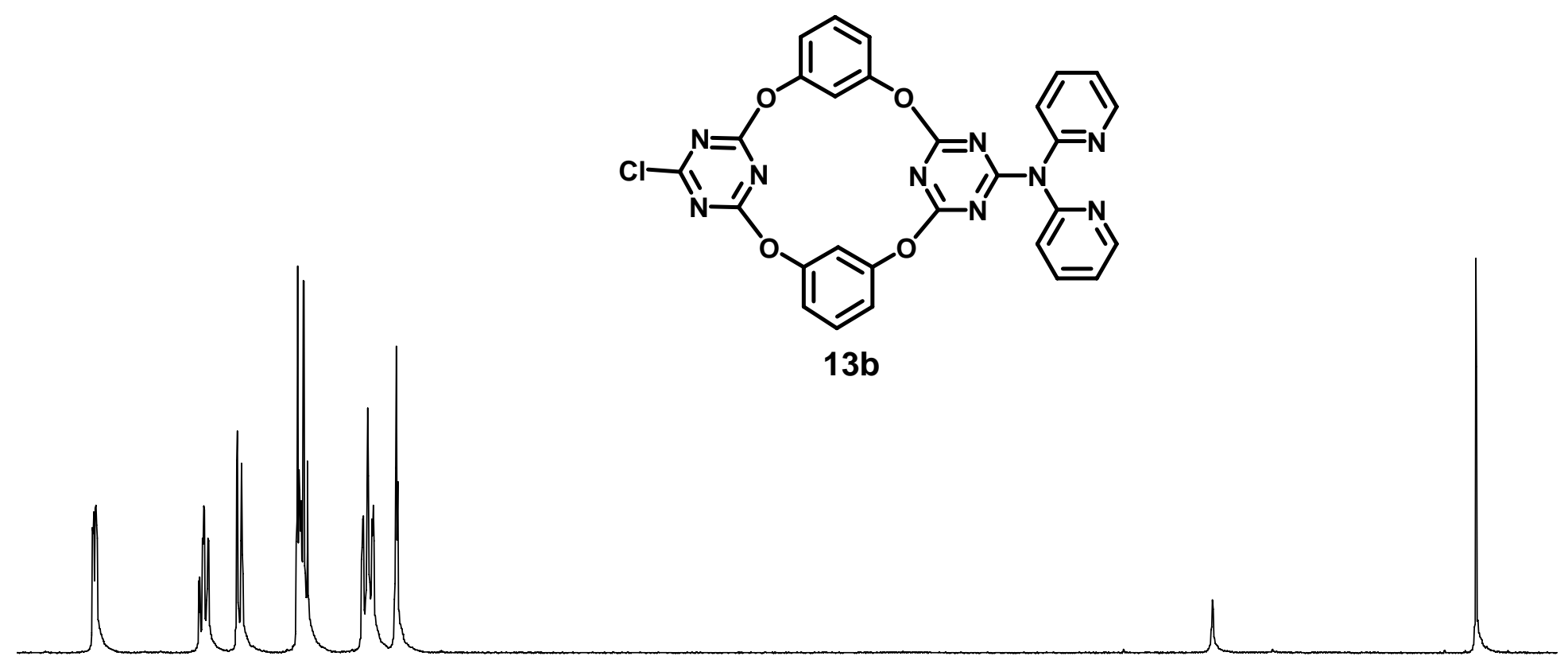

Current Data Parameters

NAME Wप93-80a

PROCNO

F2 - Acquisition Parameters

Date $\quad 20070303$

Time

PROBHD $5 \mathrm{~mm}$ DUL $13 \mathrm{C}-1$

PULPROG

$\begin{array}{ll}\text { TD } & 65536 \\ \text { SOLVENT } & \text { CDC13 }\end{array}$

DS $\quad 16$

$\begin{array}{ll}\text { SWH } & 6172.839 \mathrm{~Hz} \\ \text { FIDRES } & 0.094190 \mathrm{~Hz}\end{array}$

$\begin{array}{ll}A Q & 0.094190 \mathrm{~Hz} \\ \mathrm{AQ} & 5.3084660 \mathrm{sec}\end{array}$

DW $\quad 322.5$

$\begin{array}{ll}\text { DE } & 6.00 \text { usec } \\ \text { TE } & 6.0000 \mathrm{~K}\end{array}$

D1 $2.00000000 \mathrm{sec}$

$=======$ CHANNEL $f 1=======$
NUC1 $1 \mathrm{H}$

$\begin{array}{lr}\text { NUC1 } & 1 \mathrm{H} \text { usec } \\ \text { P1 } & 9.30 \text { use }\end{array}$

SF01 $300.1318534 \mathrm{MHz}$

F2 - Processing parameters

5I Processing parameters

SF $\quad 300.1300050 \mathrm{MHZ}$

WOW 5 SSB

$\begin{array}{lc}\text { SSB } & 0 \\ \text { LB } & 0.35 \mathrm{~Hz} \\ \text { GB } & 0 \\ \text { PC } & 1.00\end{array}$

10 NMR plot parameters

CX $22.00 \mathrm{~cm}$

$\begin{array}{lr}\text { CY } & 6.00 \mathrm{~cm} \\ \text { F1P } & 9.000 \mathrm{ppm}\end{array}$

$\begin{array}{ll}F 1 & 2701.17 \mathrm{~Hz} \\ \text { F2P } & -0.500 \mathrm{pP}\end{array}$

F2 $\quad-0.500 \mathrm{pp}$

$\begin{array}{ll}\text { PPMCM } & 0.43182 \mathrm{ppm} / \mathrm{cm} \\ \text { HZCM } & 129.60159 \mathrm{~Hz} / \mathrm{cm}\end{array}$

ppm

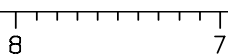

$\begin{array}{lll}7+1 & 1+1+11 & 1\end{array}$

5

4

3

2

1 

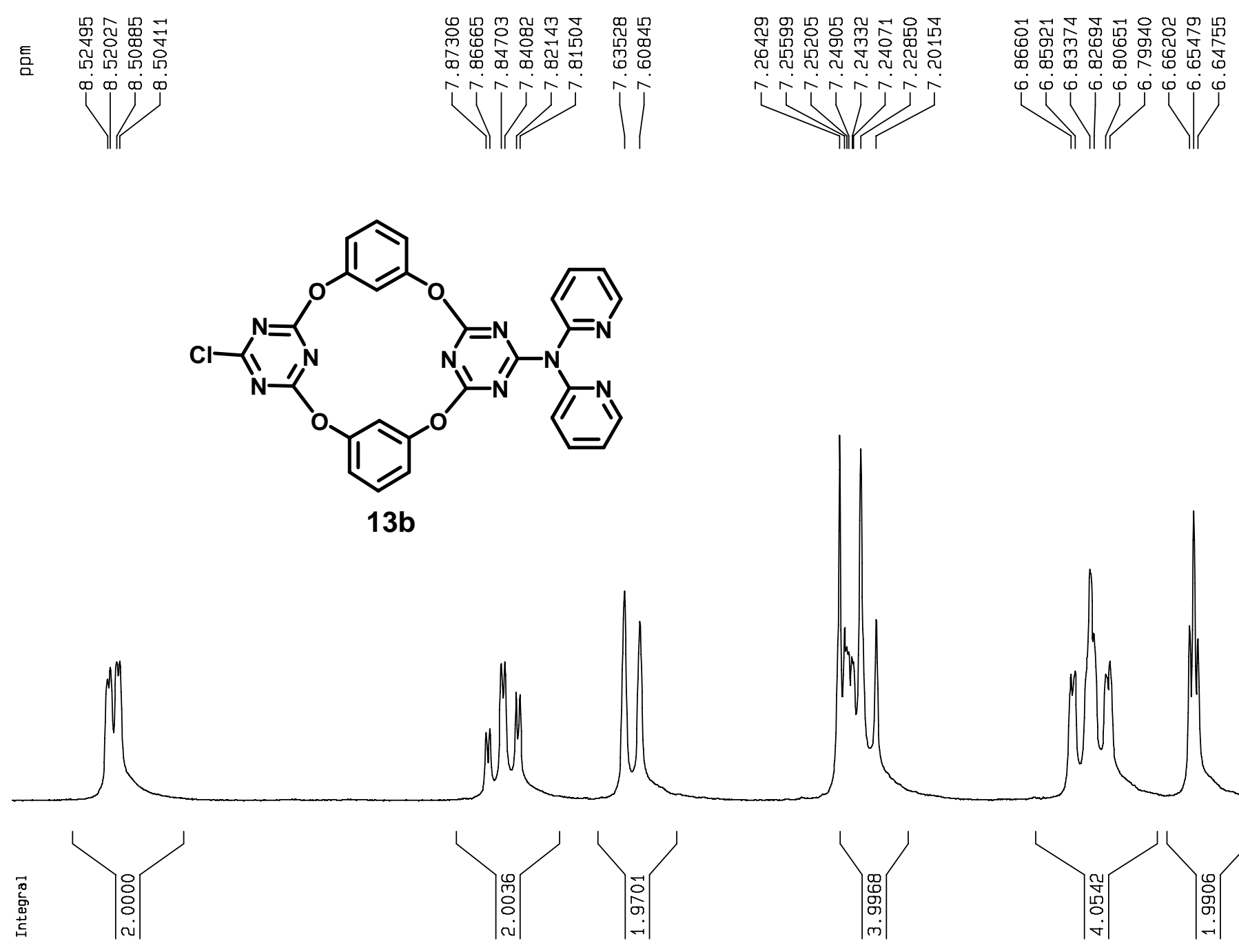

ppm $\quad 8.5$

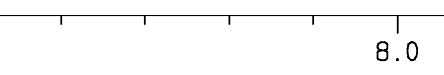

8.0

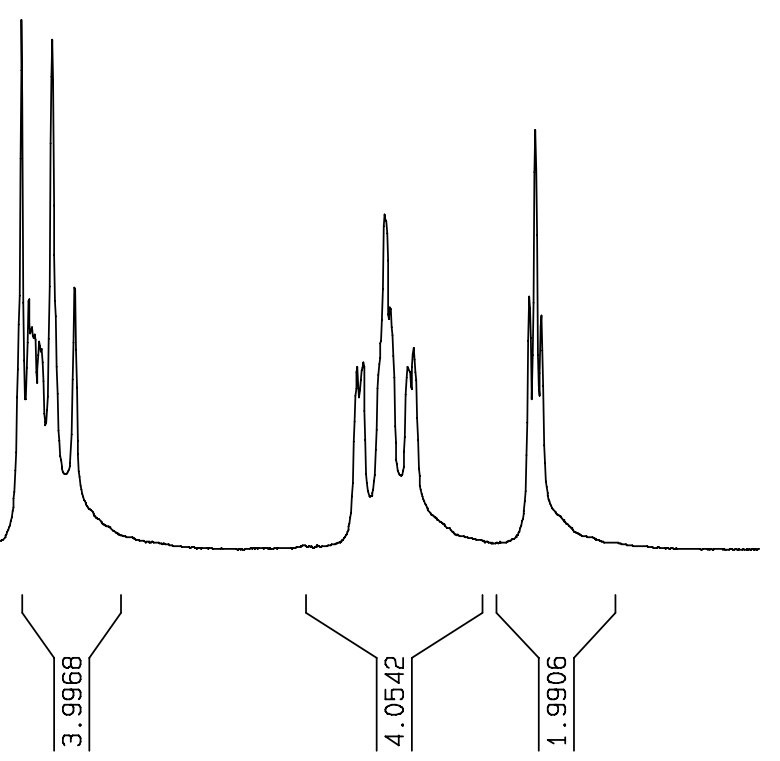

Current Data Parameters

NAME wq93-80a

EXPNO

F2 - Acquisition Parameters

Date_ 20070303

Time

INSTRUM

PROBHD $5 \mathrm{~mm}$ DUL $13 \mathrm{C}-1$

PULPROG Zg30

TD $\quad 65536$

DS

5WH $\quad 2172.839 \mathrm{~Hz}$

FIDRES $\quad 0.094190 \mathrm{~Hz}$

AQ $\quad 5.3084660 \mathrm{sec}$

RG $\quad 322.5$

$\begin{array}{lr} & \\ \text { DE } & 61.000 \text { usec } \\ \text { DE } & 6.00 \text { usec }\end{array}$

$\begin{array}{cc}\mathrm{TE} & 296.6 \mathrm{~K} \\ \mathrm{TI} & 2.00000000 \mathrm{sec}\end{array}$

$=======$ CHANNEL $f 1$ = $======$

$\begin{array}{lr}\text { NUC1 } & 1 \mathrm{H} \\ \mathrm{P} 1 & 9.30 \text { use }\end{array}$

$\begin{array}{lr}\text { P1 } & 9.30 \text { usec } \\ \text { Ph1 } & -1.000 \text { dB }\end{array}$

$\begin{array}{lr}\text { PL1 } & -1.00 \mathrm{~dB} \\ \text { SF01 } & 300.1318534 \mathrm{MHz}\end{array}$

F2 - Processing parameters

SI 32768

SF $\quad 300.1300050 \mathrm{MHz}$

WDW

5SB
LB

GB
PC

$$
\begin{gathered}
\text { EM } \\
0 \\
0.35 \mathrm{~Hz} \\
0 \\
1.00
\end{gathered}
$$

10 NMB plot parameters

CX $\quad 22.00 \mathrm{~cm}$

$\begin{array}{ll}C Y & 6.00 \mathrm{~cm}\end{array}$

$\begin{array}{lr}F 1 & 8.690 \mathrm{pp} \\ \mathrm{F} 1 & 2608.07 \mathrm{~Hz}\end{array}$

F2P $\quad 5.389 \mathrm{~Hz}$

F2 $1917.66 \mathrm{~Hz}$

PPMCM $\quad 0.10456 \mathrm{ppm} / \mathrm{cm}$

HZCM

$31.38242 \mathrm{~Hz} / \mathrm{cm}$ 
${ }^{13} \mathrm{C}$ NMR of 13b
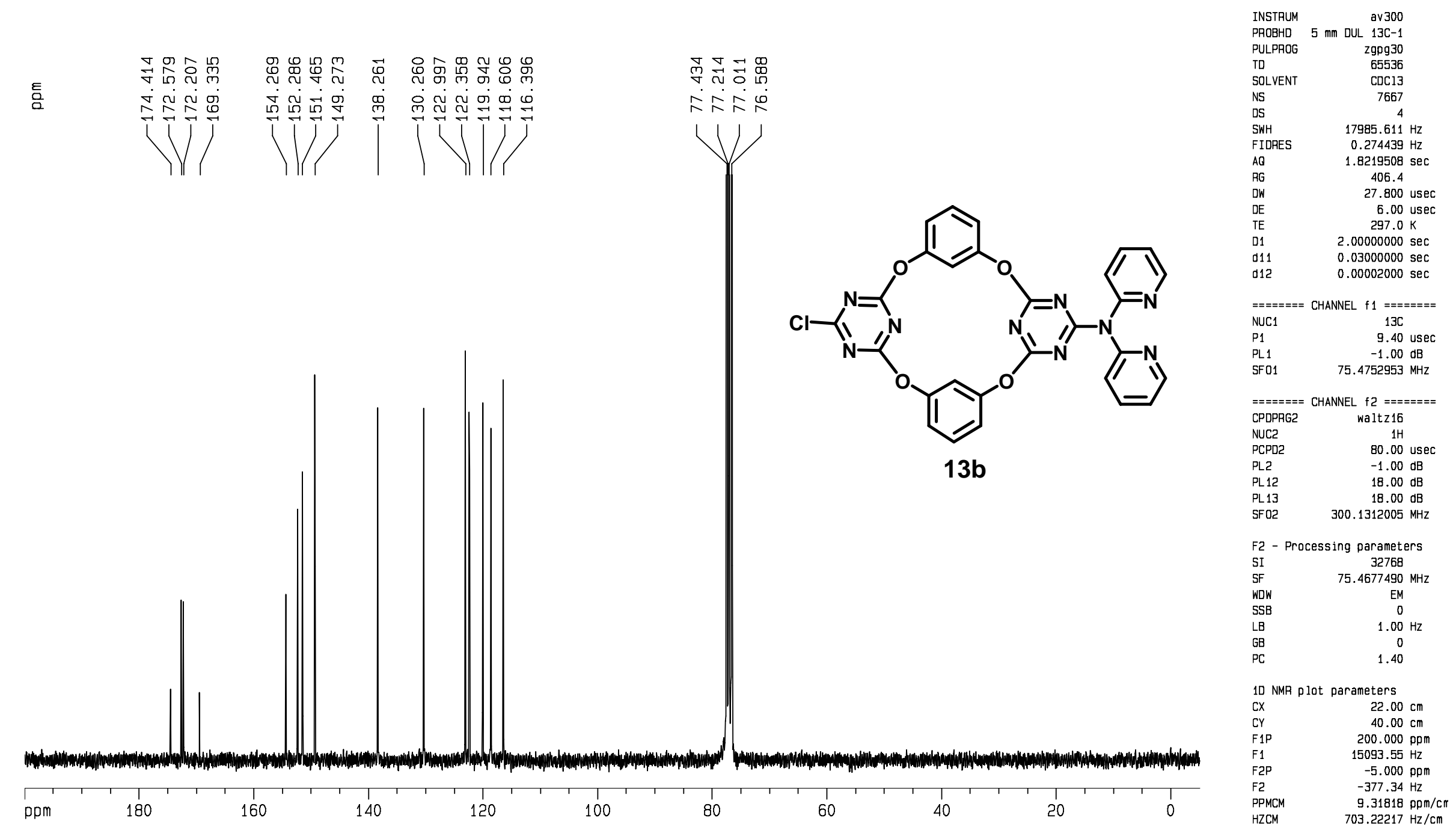


\section{${ }^{1} \mathrm{H}$ NMR of $\mathbf{1 3 c}$}

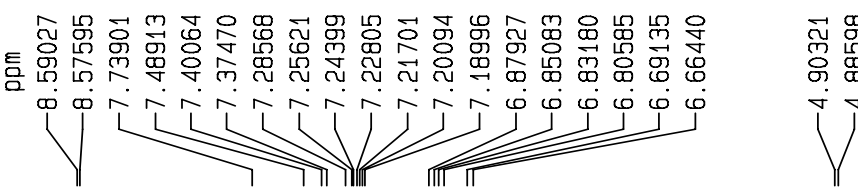
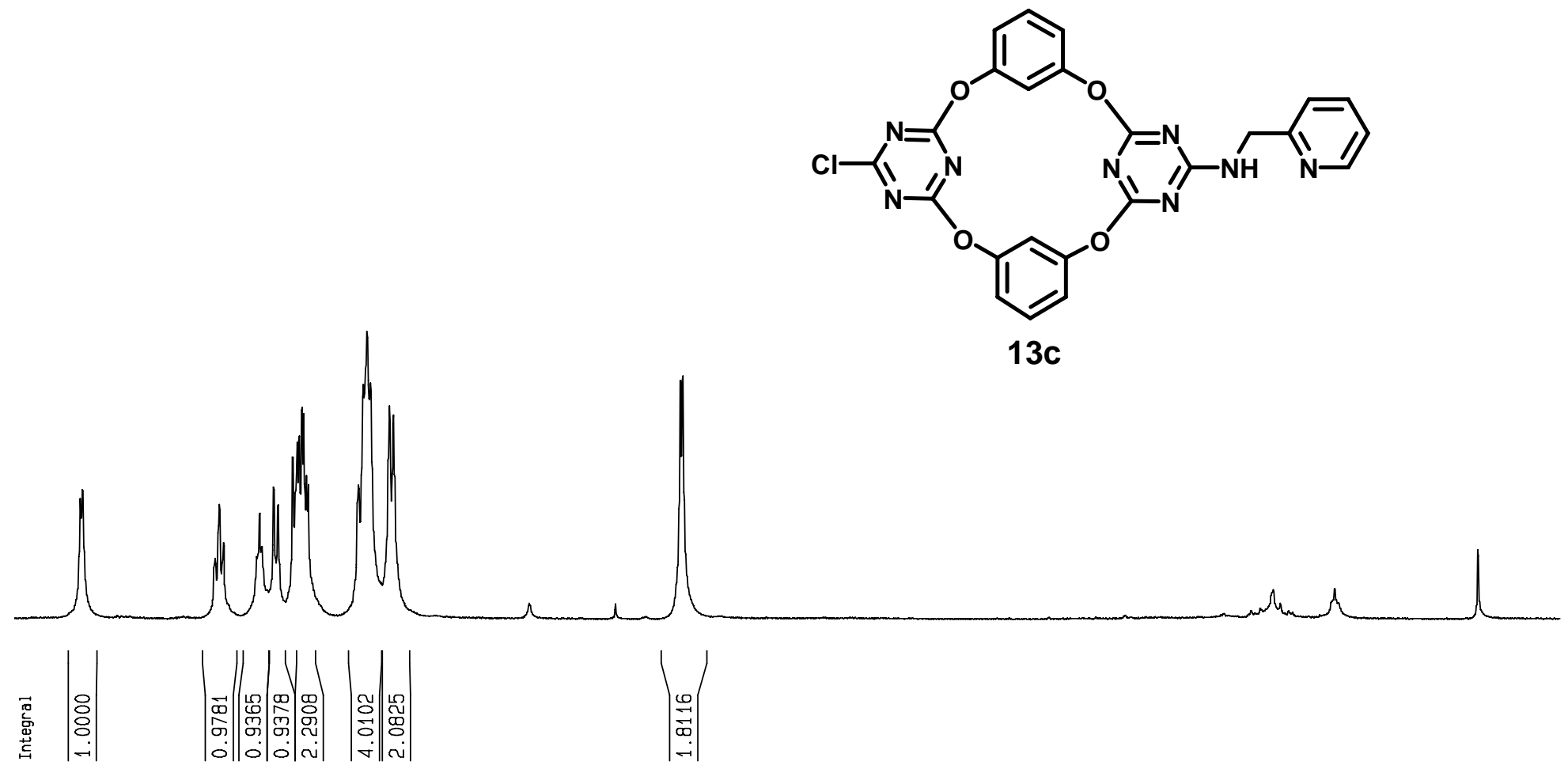

Current Data Parameters

NAME

ROCNO

พqव -78 a
20

F2 - Acquisition Parameters

Date

20070208

INSTRUM

PULPROG zg30

DS

2
5 WH

FIDRES $\quad 0.094190 \mathrm{~Hz}$

$\quad 5.3084660 \mathrm{sec}$

DW 81.000 use

DE $\quad 6.00$ usec

$2.00000000 \mathrm{sec}$

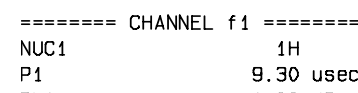

$\begin{array}{lr}\text { P1 } & 9.30 \text { use } \\ \text { PL1 } & -1.00 \mathrm{~dB}\end{array}$

$300.1318534 \mathrm{MHz}$

F2 - Processing parameters

$\begin{array}{cc}\mathrm{SI} & 32768 \\ \mathrm{SF} & 300.1299982 \mathrm{MHz}\end{array}$

WDW $\quad$ EM

$\begin{array}{lc}\text { SSB } & 0 \\ \text { LB } & 0.35 \mathrm{~Hz} \\ \text { GB } & 0 \\ \text { PC } & 1.00\end{array}$

10 NMR plot parameters

$\begin{array}{ll}\text { CX } & 22.00 \mathrm{~cm}\end{array}$

$\begin{array}{lr}\text { CY } & 4.50 \mathrm{~cm} \\ \text { F1P } & 9.000 \mathrm{ppm} \\ \text { F1 } & 270.17 \mathrm{~Hz}\end{array}$

$\begin{array}{ll}F 1 & 2701.17 \mathrm{~Hz} \\ \text { F2P } & -0.500\end{array}$

F2 $\quad-150.07 \mathrm{~Hz}$

$\begin{array}{lr}\text { HZCM } & 0.43182 \mathrm{ppm} / \mathrm{cm} \\ & 129.60159 \mathrm{~Hz} / \mathrm{cm}\end{array}$ 

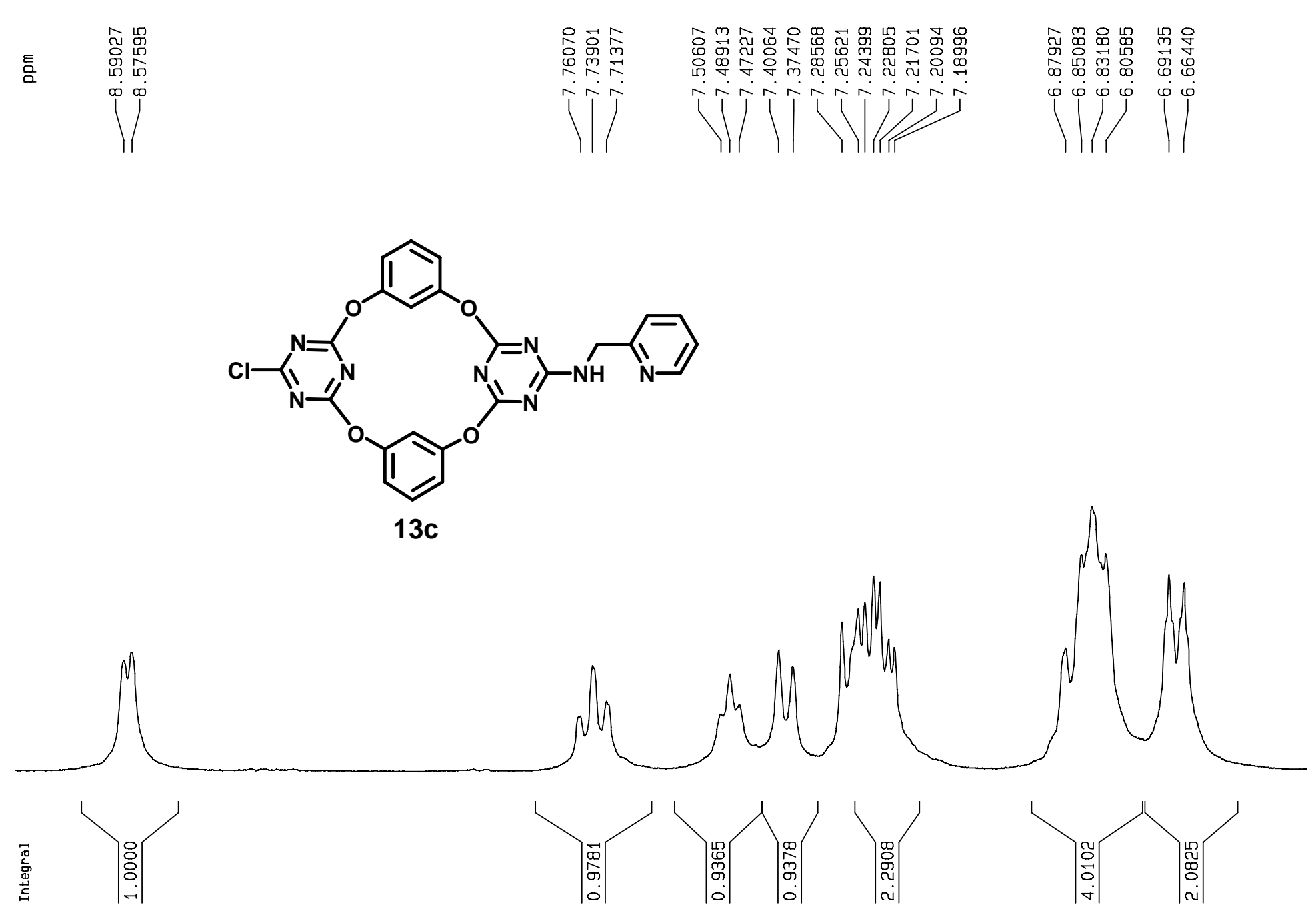
${ }^{13} \mathrm{C}$ NMR of $\mathbf{1 3 c}$
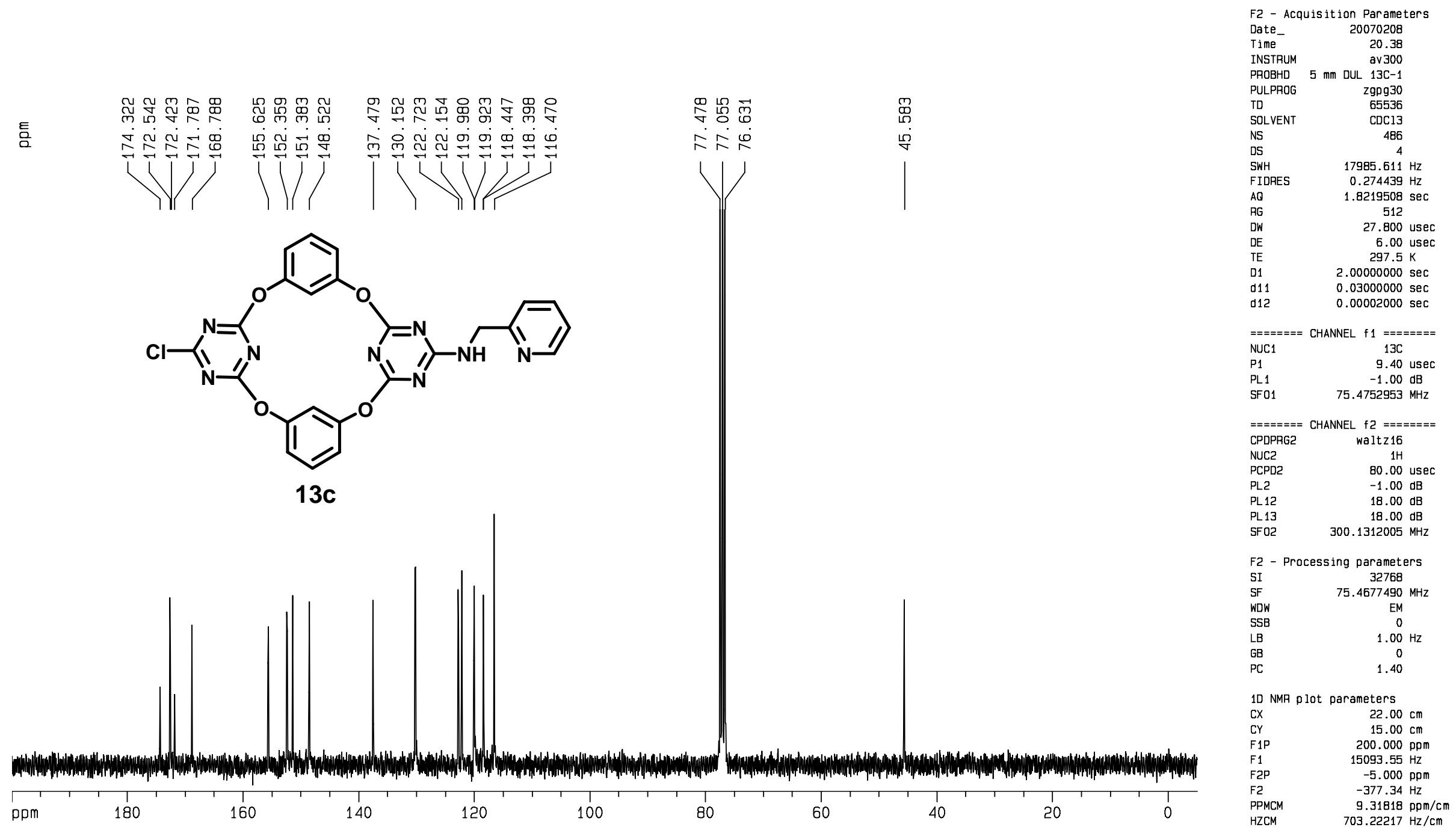


\section{UV-vis Titration of 12a-c with $\mathrm{Cu}^{2+}, \mathrm{Fe}^{2+}, \mathrm{Co}^{2+}$, $\mathrm{Ni}^{2+}, \mathrm{Zn}^{2+}$ and 13a-c with $\mathrm{Cu}^{2+}$}

( Perchlorate salts were used as metal ion sources unless indicated)

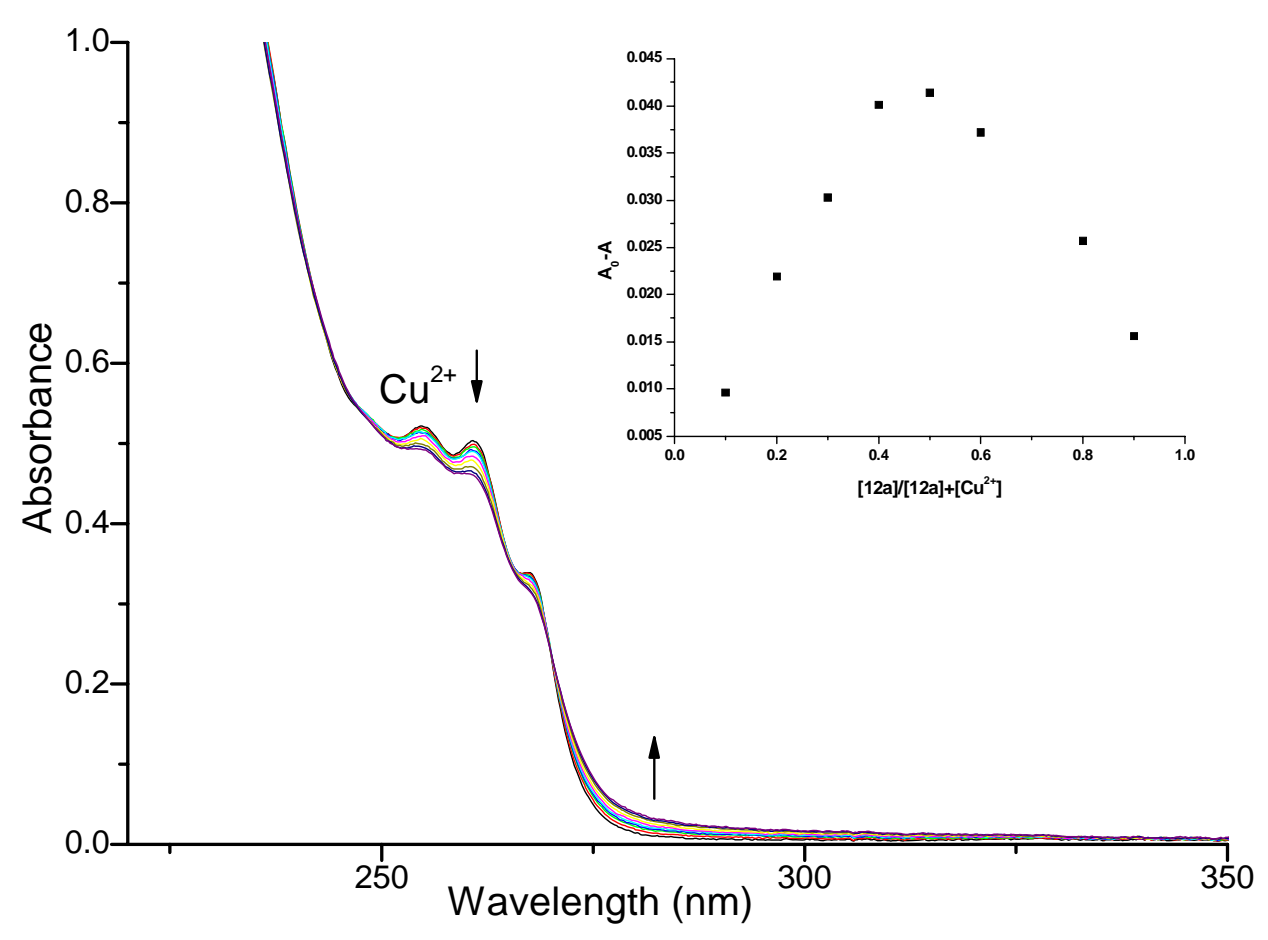

Figure S1. UV-vis titration of $12 \mathrm{a}\left(1.728 \times 10^{-5} \mathrm{~mol} \cdot \mathrm{L}^{-1}\right)$ upon the addition of $\mathrm{Cu}^{2+}$ in a mixture of acetonitrile and water $(4: 1)$. The concentrations of $\mathrm{Cu}^{2+}$ are $0,0.766,1.53,2.30,3.06,4.60,7.66,13.79$, 22.98, $32.17\left(\times 10^{-5} \mathrm{~mol} \cdot \mathrm{L}^{-1}\right)$, respectively. Insert is the Job plot of the complexation between 12a and $\mathrm{Cu}^{2+}$ ion in a mixture of acetonitrile and water $(4: 1)$, and the total concentration of $12 \mathbf{a}$ and $\mathrm{Cu}^{2+}$ is $8 \times 10^{-5}$ $\mathrm{mol} \cdot \mathrm{L}^{-1}$. 


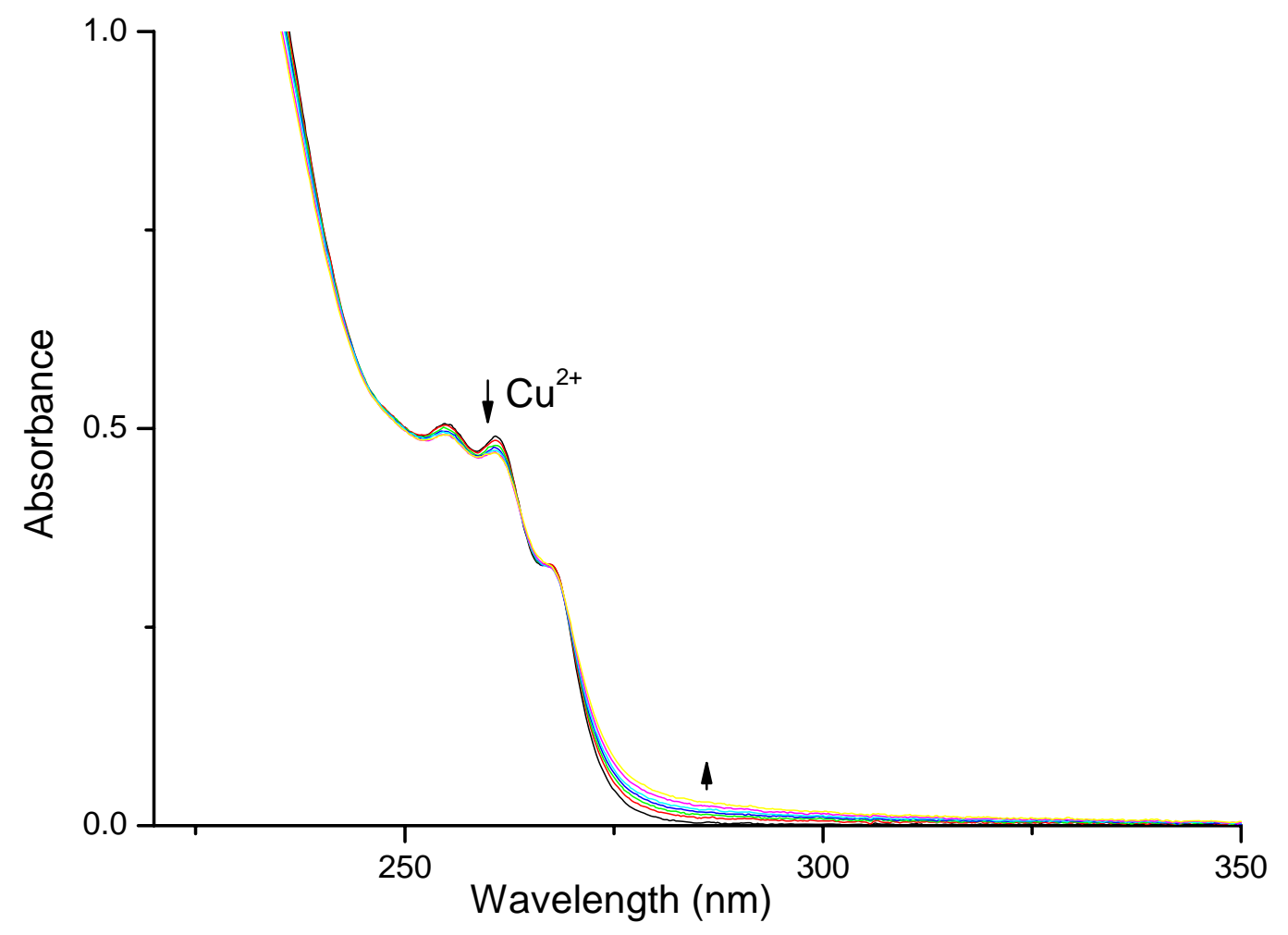

Figure S2. UV-vis titration of 12a $\left(1.728 \times 10^{-5} \mathrm{~mol} \cdot \mathrm{L}^{-1}\right)$ upon the addition of $\mathrm{CuCl}_{2}$ in a mixture of acetonitrile and water $(4: 1)$. The concentrations of $\mathrm{CuCl}_{2}$ are $0,0.773,1.55,2.32,3.09,4.64,6.18\left(\times 10^{-5}\right.$ mol. $\left.\cdot \mathrm{L}^{-1}\right)$, respectively.

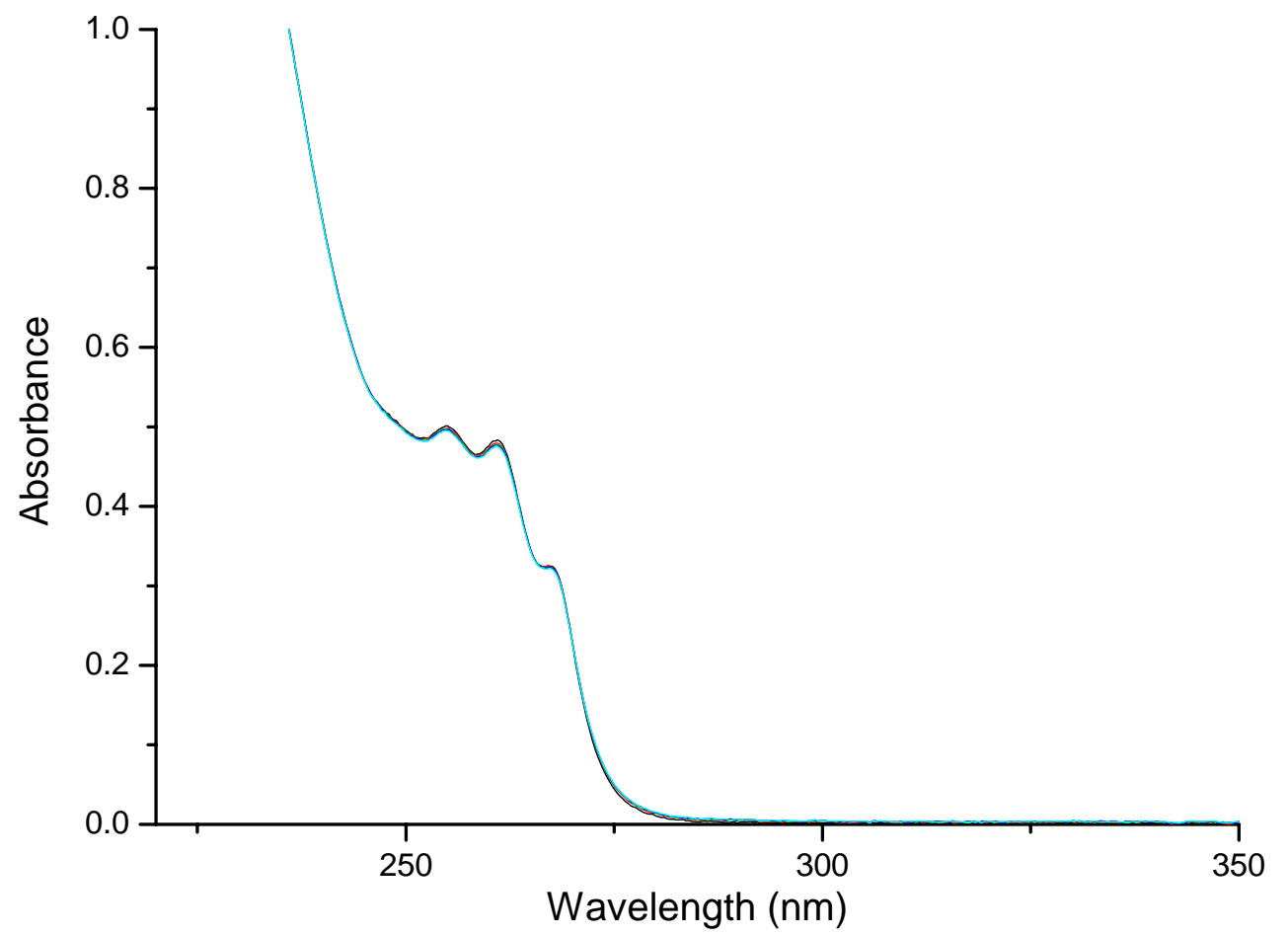

Figure S3. UV-vis titration of $12 \mathrm{a}\left(1.728 \times 10^{-5} \mathrm{~mol} \cdot \mathrm{L}^{-1}\right)$ upon the addition of $\mathrm{Fe}^{2+}$ in a mixture of acetonitrile and water $(4: 1)$. The concentrations of $\mathrm{Fe}^{2+}$ are $0,0.836,1.67,2.51,3.34,4.18\left(\times 10^{-5} \mathrm{~mol} \cdot \mathrm{L}^{-1}\right)$, respectively. 


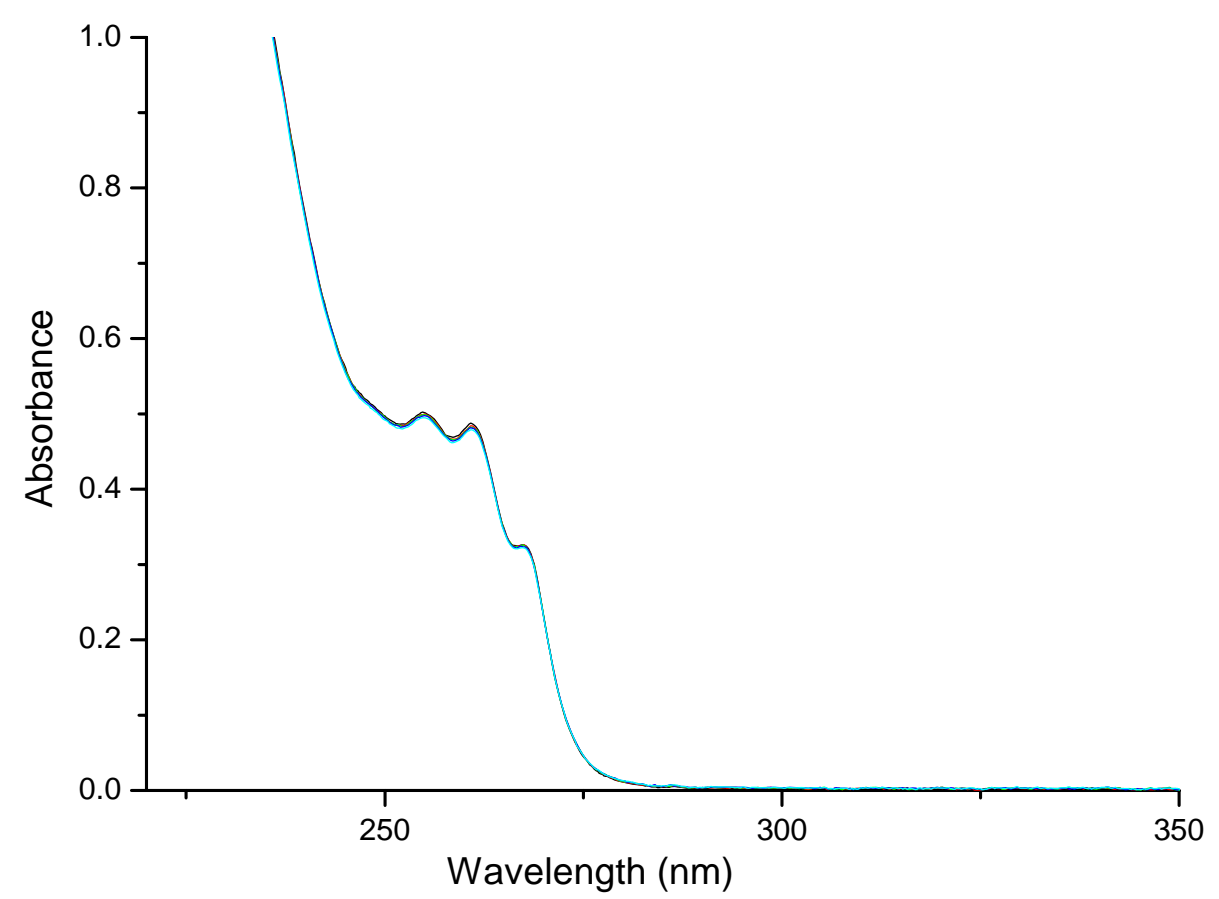

Figure S4. UV-vis titration of 12a $\left(1.728 \times 10^{-5} \mathrm{~mol} \cdot \mathrm{L}^{-1}\right)$ upon the addition of $\mathrm{Co}^{2+}$ in a mixture of

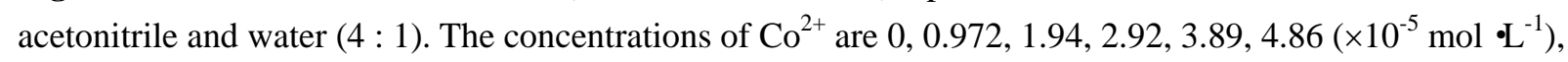
respectively.

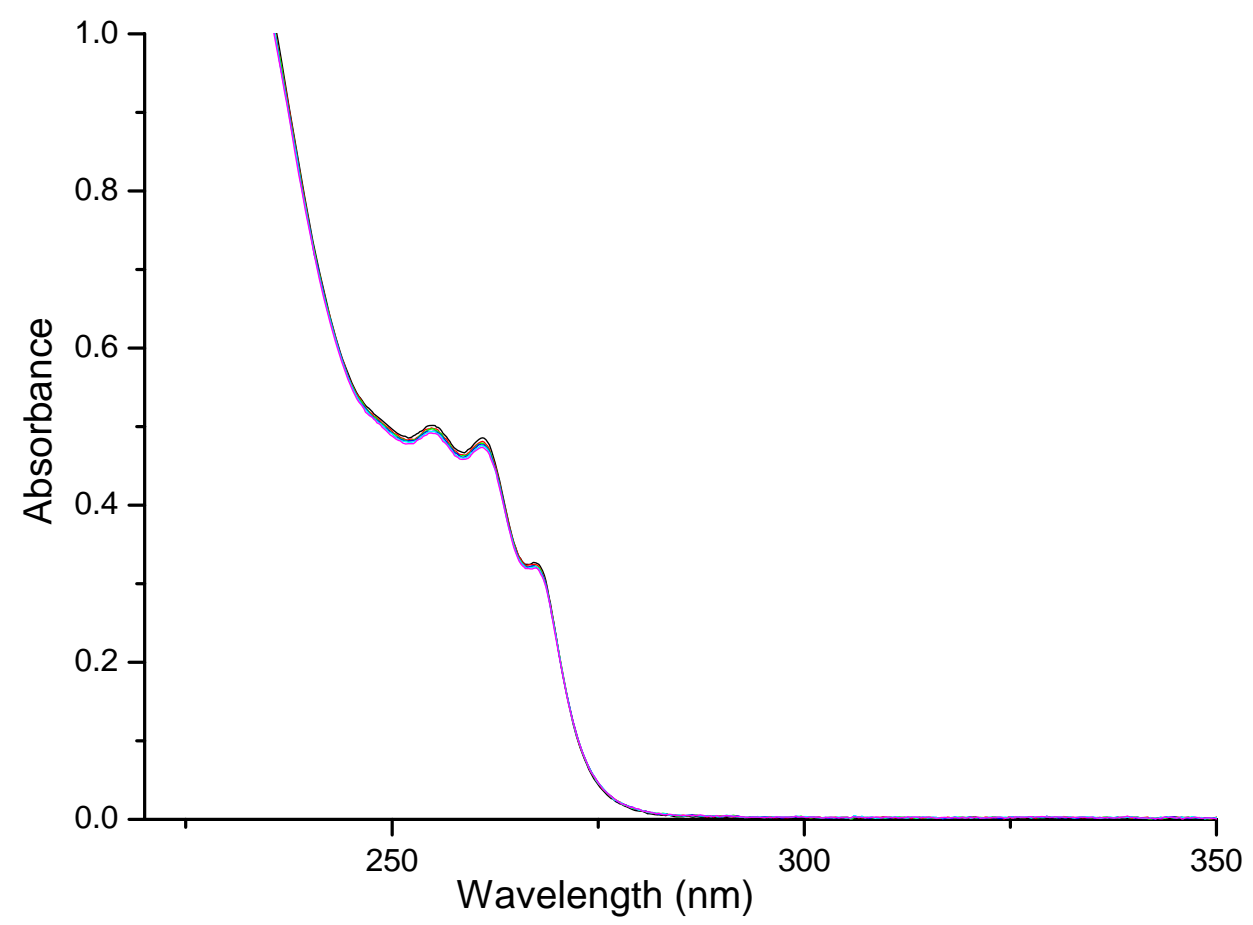

Figure S5. UV-vis titration of $12 \mathrm{a}\left(1.728 \times 10^{-5} \mathrm{~mol} \cdot \mathrm{L}^{-1}\right)$ upon the addition of $\mathrm{Ni}^{2+}$ in a mixture of acetonitrile and water $(4: 1)$. The concentrations of $\mathrm{Ni}^{2+}$ are $0,0.864,1.73,2.59,3.45,4.32\left(\times 10^{-5} \mathrm{~mol} \cdot \mathrm{L}^{-1}\right)$, respectively. 


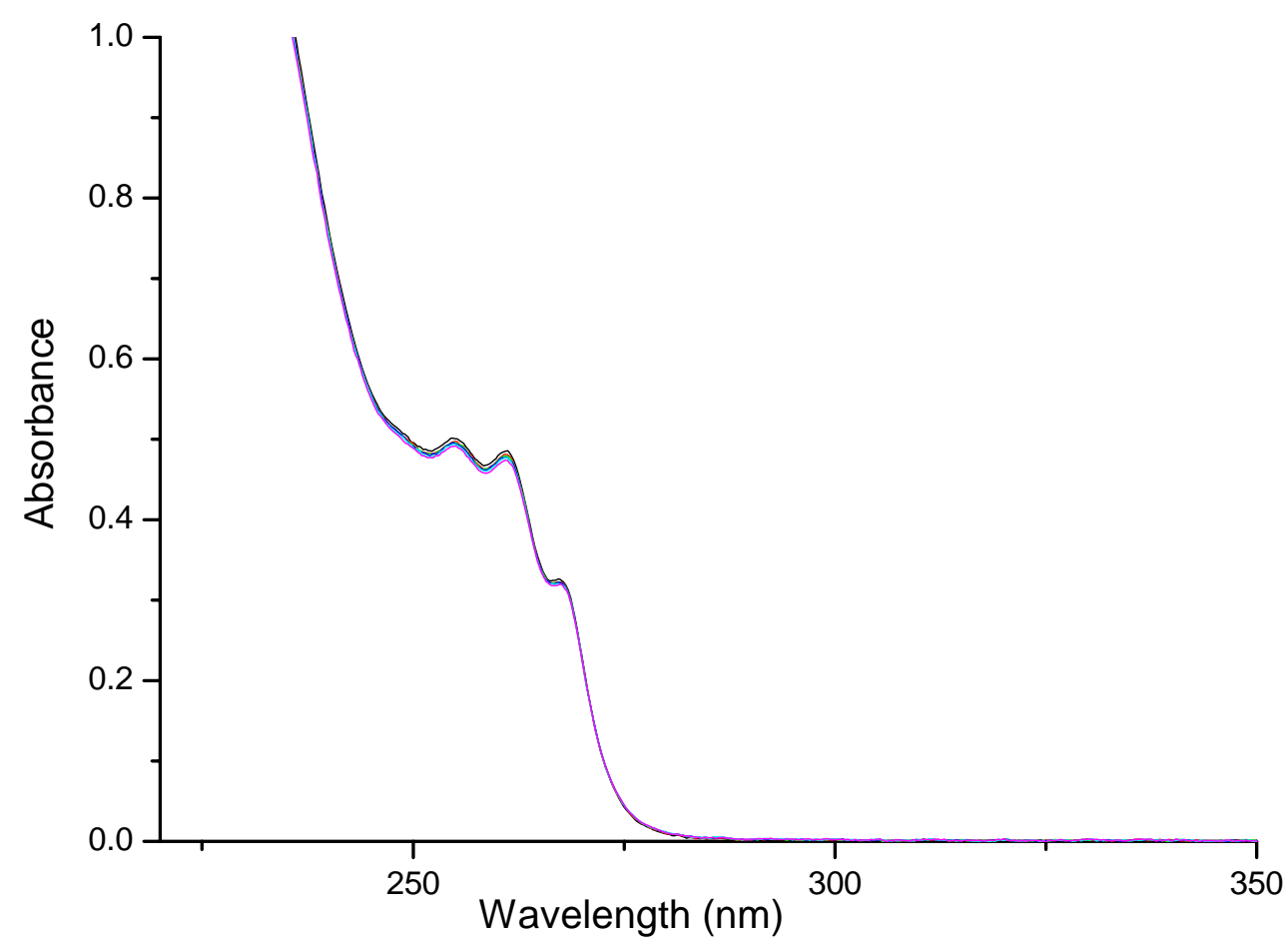

Figure S6. UV-vis titration of $12 \mathrm{a}\left(1.728 \times 10^{-5} \mathrm{~mol} \cdot \mathrm{L}^{-1}\right)$ upon the addition of $\mathrm{Zn}^{2+}$ in a mixture of acetonitrile and water $(4: 1)$. The concentrations of $\mathrm{Zn}^{2+}$ are $0,0.828,1.66,2.48,3.31,4.14\left(\times 10^{-5} \mathrm{~mol} \cdot \mathrm{L}^{-1}\right)$, respectively.

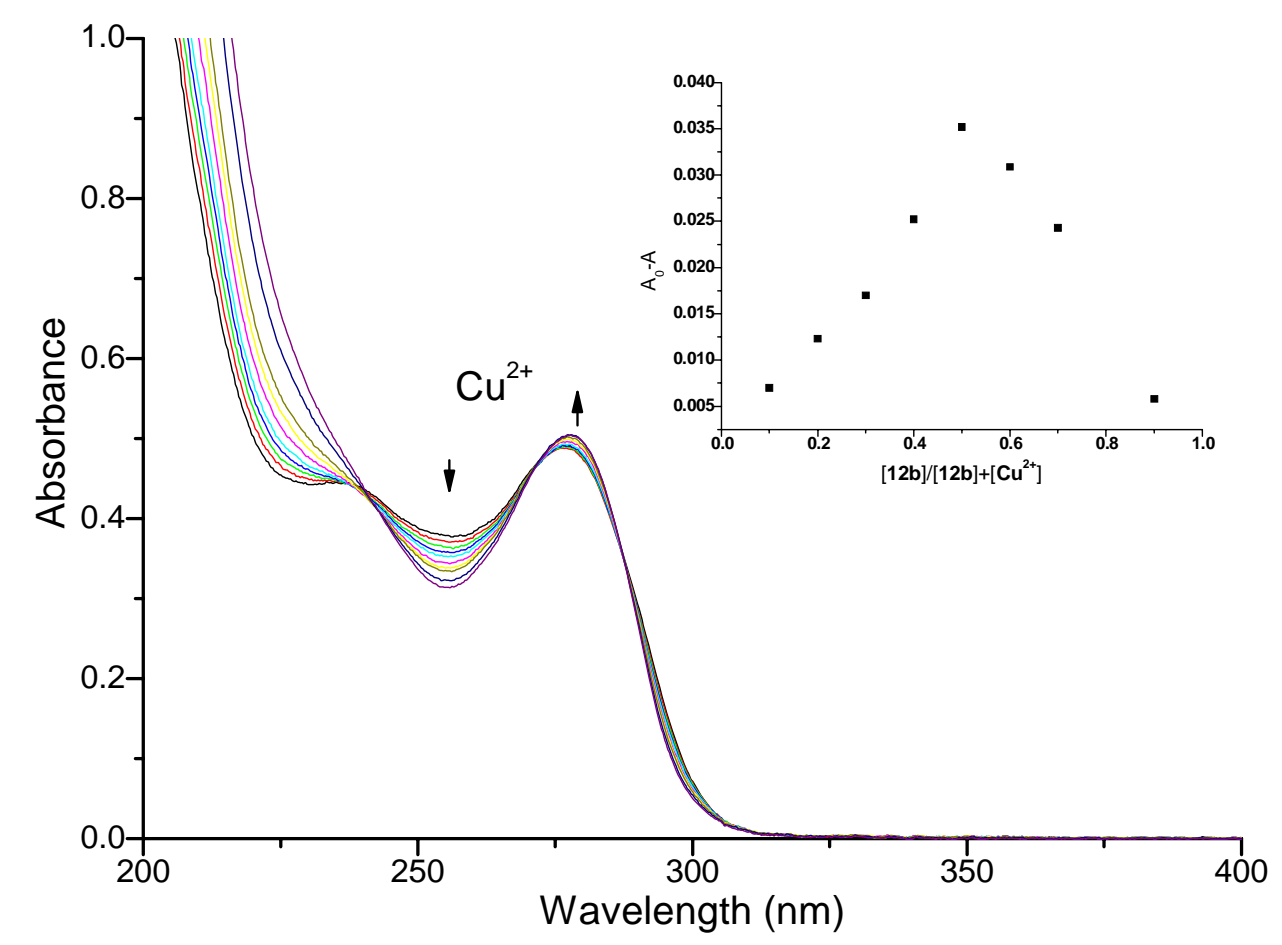

Figure S7. UV-vis titration of $\mathbf{1 2 b}\left(1.194 \times 10^{-5} \mathrm{~mol} \cdot \mathrm{L}^{-1}\right)$ upon the addition of $\mathrm{Cu}^{2+}$ in a mixture of acetonitrile and water $(4: 1)$. The concentrations of $\mathrm{Cu}^{2+}$ are $0,0.766,1.53,2.30,3.06,4.60,6.13,7.66$, 13.79, $19.92\left(\times 10^{-5} \mathrm{~mol} \cdot \mathrm{L}^{-1}\right)$, respectively. Insert is the Job plot of the complexation between $\mathbf{1 2} \mathbf{b}$ and $\mathrm{Cu}^{2+}$ ion in a mixture of acetonitrile and water ( $4: 1$ ), and the total concentration of $\mathbf{1 2} \mathbf{b}$ and $\mathrm{Cu}^{2+}$ is $4 \times 10^{-5}$ $\mathrm{mol} \cdot \mathrm{L}^{-1}$. 


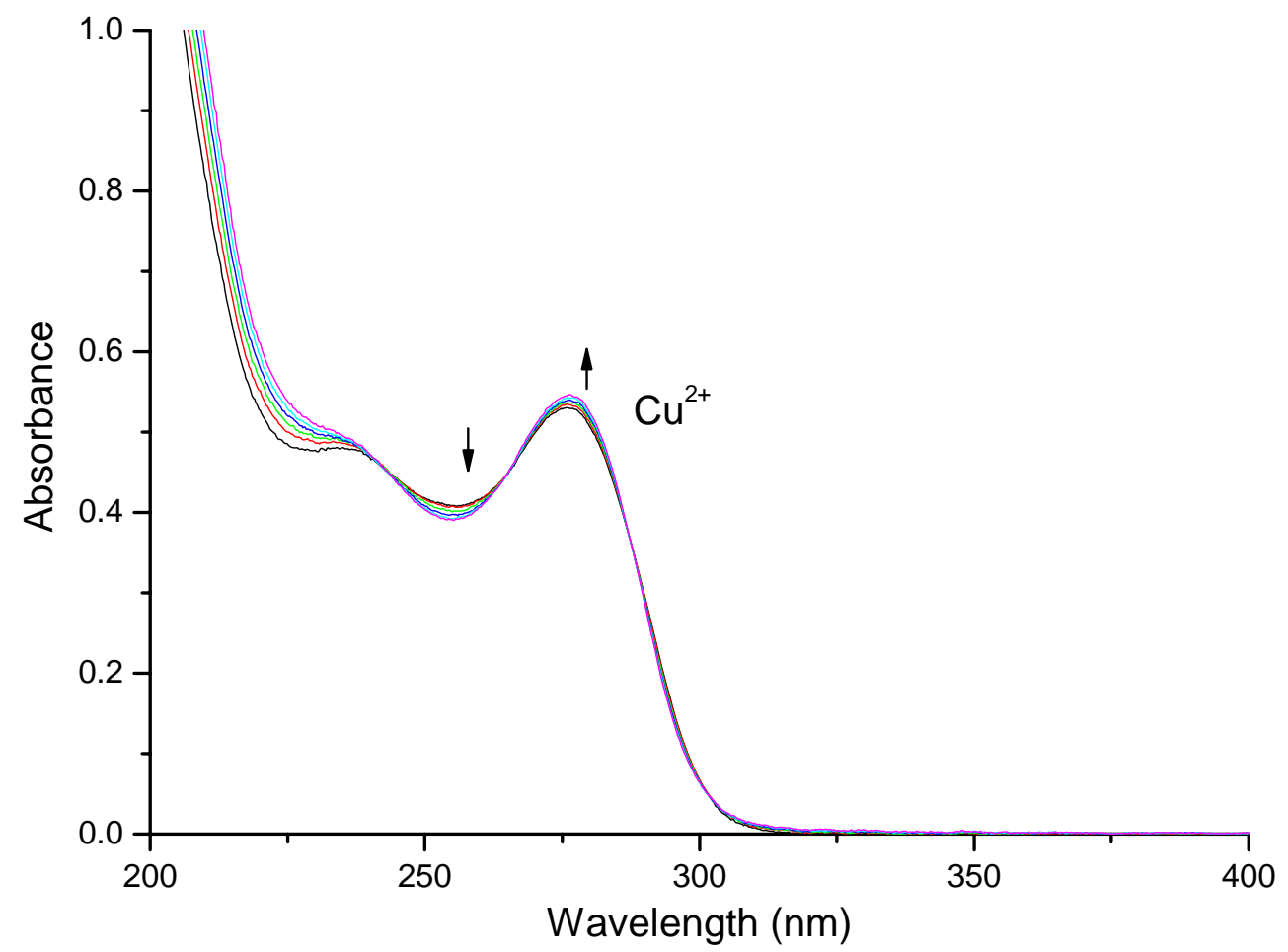

Figure S8. UV-vis titration of $\mathbf{1 2 b}\left(1.194 \times 10^{-5} \mathrm{~mol} \cdot \mathrm{L}^{-1}\right)$ upon the addition of $\mathrm{CuCl}_{2}$ in a mixture of acetonitrile and water $(4: 1)$. The concentrations of $\mathrm{CuCl}_{2}$ are $0,0.796,1.59,2.39,3.18,3.98\left(\times 10^{-5}\right.$ $\left.\mathrm{mol} \cdot \mathrm{L}^{-1}\right)$, respectively.

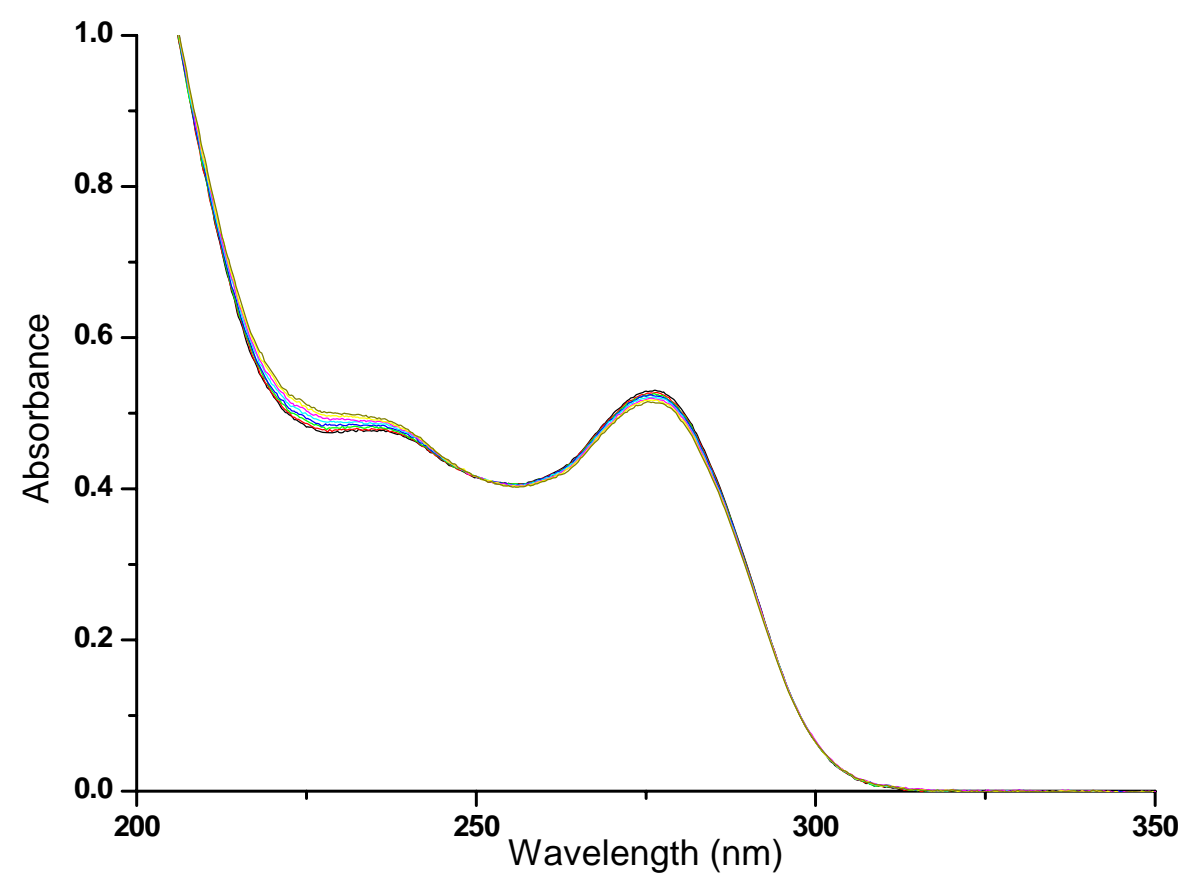

Figure S9. UV-vis titration of $\mathbf{1 2 b}\left(1.194 \times 10^{-5} \mathrm{~mol} \cdot \mathrm{L}^{-1}\right)$ upon the addition of $\mathrm{Fe}^{2+}$ in a mixture of acetonitrile and water $(4: 1)$. The concentrations of $\mathrm{Fe}^{2+}$ are $0,0.836,1.67,2.51,3.76,5.02,6.27,7.52$ $\left(\times 10^{-5} \mathrm{~mol} \cdot \mathrm{L}^{-1}\right)$, respectively. 


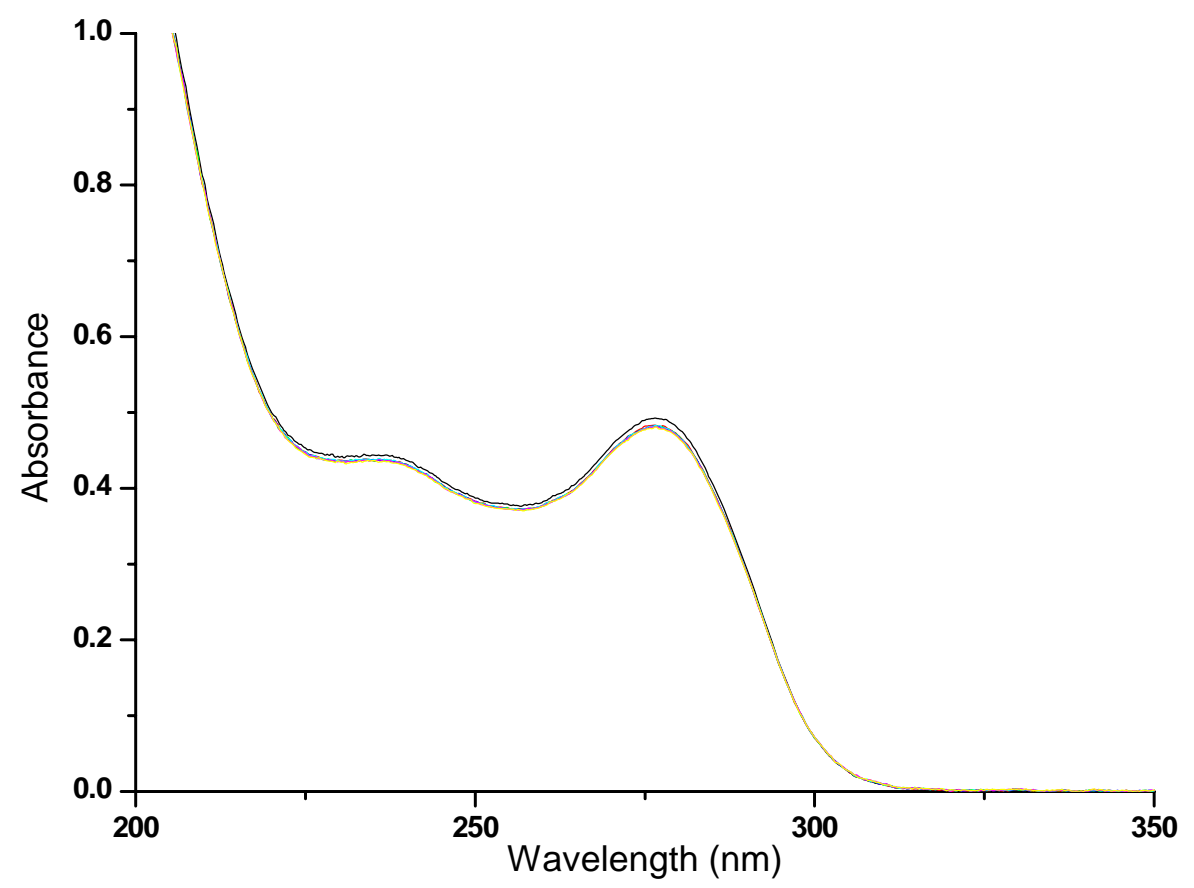

Figure S10. UV-vis titration of $\mathbf{1 2 b}\left(1.194 \times 10^{-5} \mathrm{~mol} \cdot \mathrm{L}^{-1}\right)$ upon the addition of $\mathrm{Co}^{2+}$ in a mixture of acetonitrile and water $(4: 1)$. The concentrations of $\mathrm{Co}^{2+}$ are $0,0.486,0.972,1.46,1.94,2.92,3.89\left(\times 10^{-5}\right.$ $\left.\mathrm{mol} \cdot \mathrm{L}^{-1}\right)$, respectively.

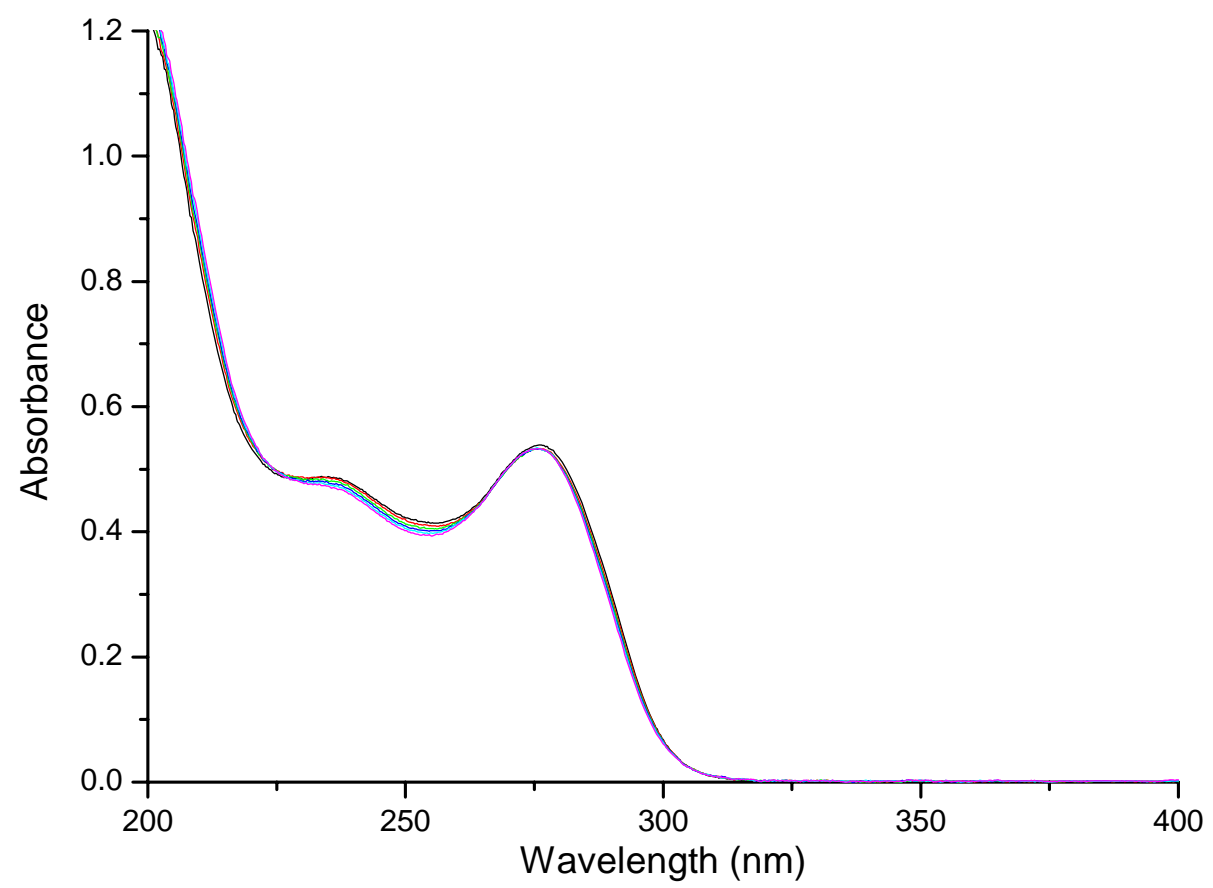

Figure S11. UV-vis titration of $\mathbf{1 2 b}\left(1.194 \times 10^{-5} \mathrm{~mol} \cdot \mathrm{L}^{-1}\right)$ upon the addition of $\mathrm{Ni}^{2+}$ in a mixture of acetonitrile and water $(4: 1)$. The concentrations of $\mathrm{Ni}^{2+}$ are $0,0.862,1.724,2.59,3.45,4.31\left(\times 10^{-5}\right.$ mol $\left.\cdot \mathrm{L}^{-1}\right)$, respectively. 


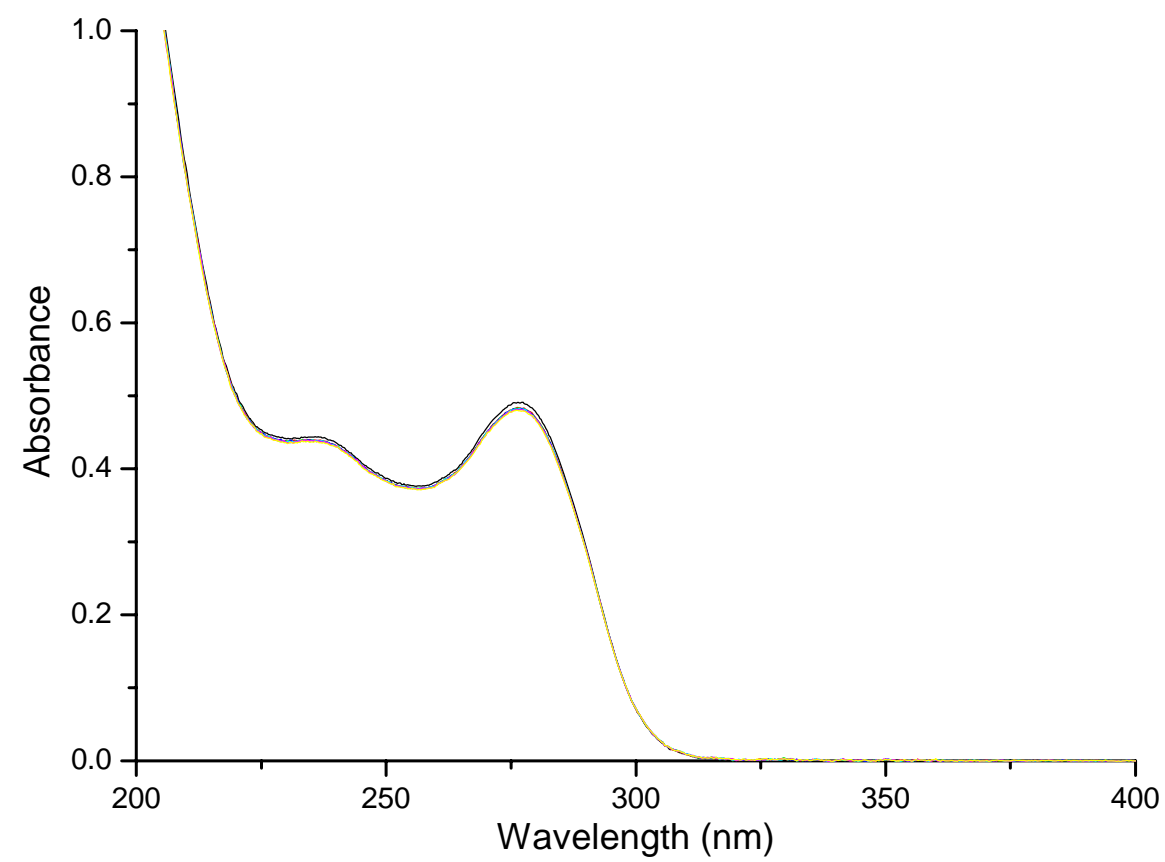

Figure S12. UV-vis titration of $\mathbf{1 2 b}\left(1.194 \times 10^{-5} \mathrm{~mol} \cdot \mathrm{L}^{-1}\right)$ upon the addition of $\mathrm{Zn}^{2+}$ in a mixture of acetonitrile and water $(4: 1)$. The concentrations of $\mathrm{Zn}^{2+}$ are $0,0.414,0.828,1.24,1.66,2.48,3.31\left(\times 10^{-5}\right.$ $\left.\mathrm{mol} \cdot \mathrm{L}^{-1}\right)$, respectively.

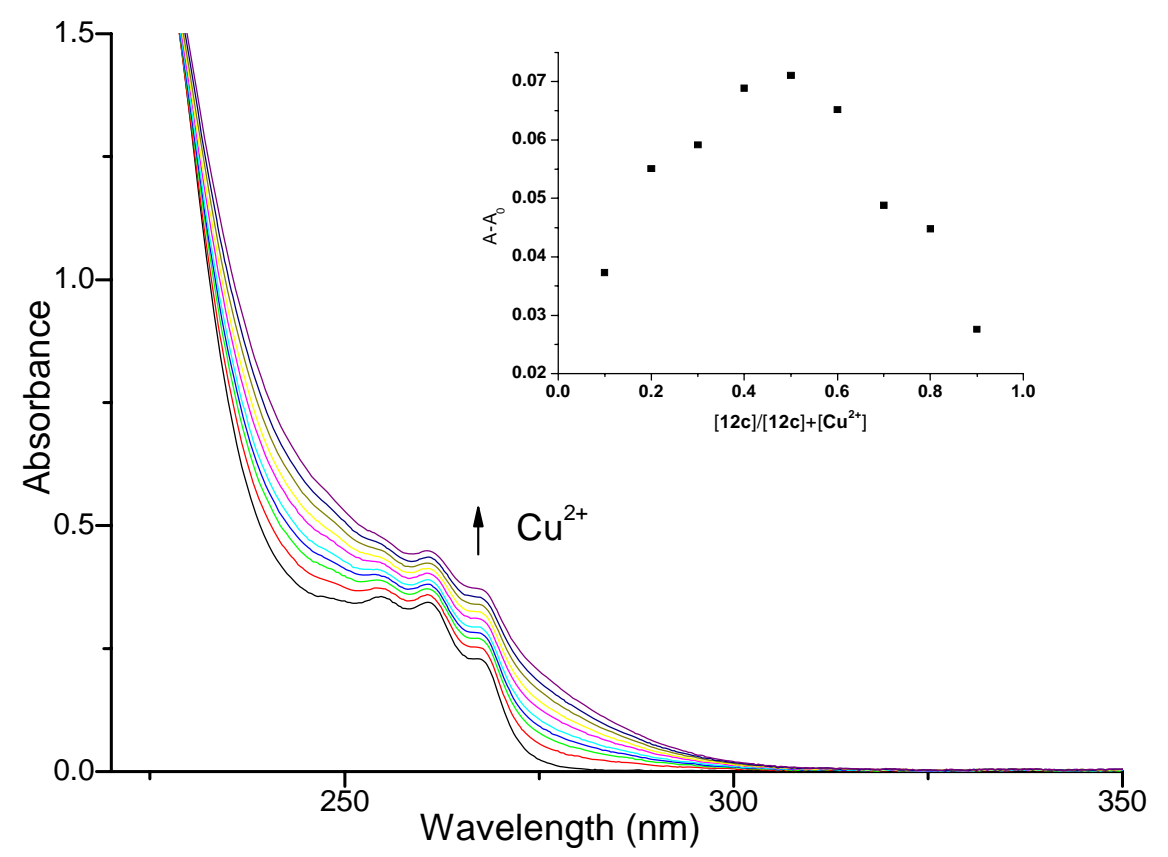

Figure S13. UV-vis titration of 12c $\left(2.386 \times 10^{-5} \mathrm{~mol} \cdot \mathrm{L}^{-1}\right)$ upon the addition of $\mathrm{Cu}^{2+}$ in a mixture of acetonitrile and water $(4: 1)$. The concentrations of $\mathrm{Cu}^{2+}$ are $0,0.552,1.10,1.66,2.21,3.31,4.42,6.62$, 8.83, $12.14\left(\times 10^{-5} \mathrm{~mol} \cdot \mathrm{L}^{-1}\right)$, respectively. Insert is the Job plot of the complexation between $12 \mathrm{c}$ and $\mathrm{Cu}^{2+}$ ion in a mixture of acetonitrile and water ( $4: 1$ ), and the total concentration of $12 \mathrm{c}$ and $\mathrm{Cu}^{2+}$ is $4 \times 10^{-5}$ $\mathrm{mol} \cdot \mathrm{L}^{-1}$. 


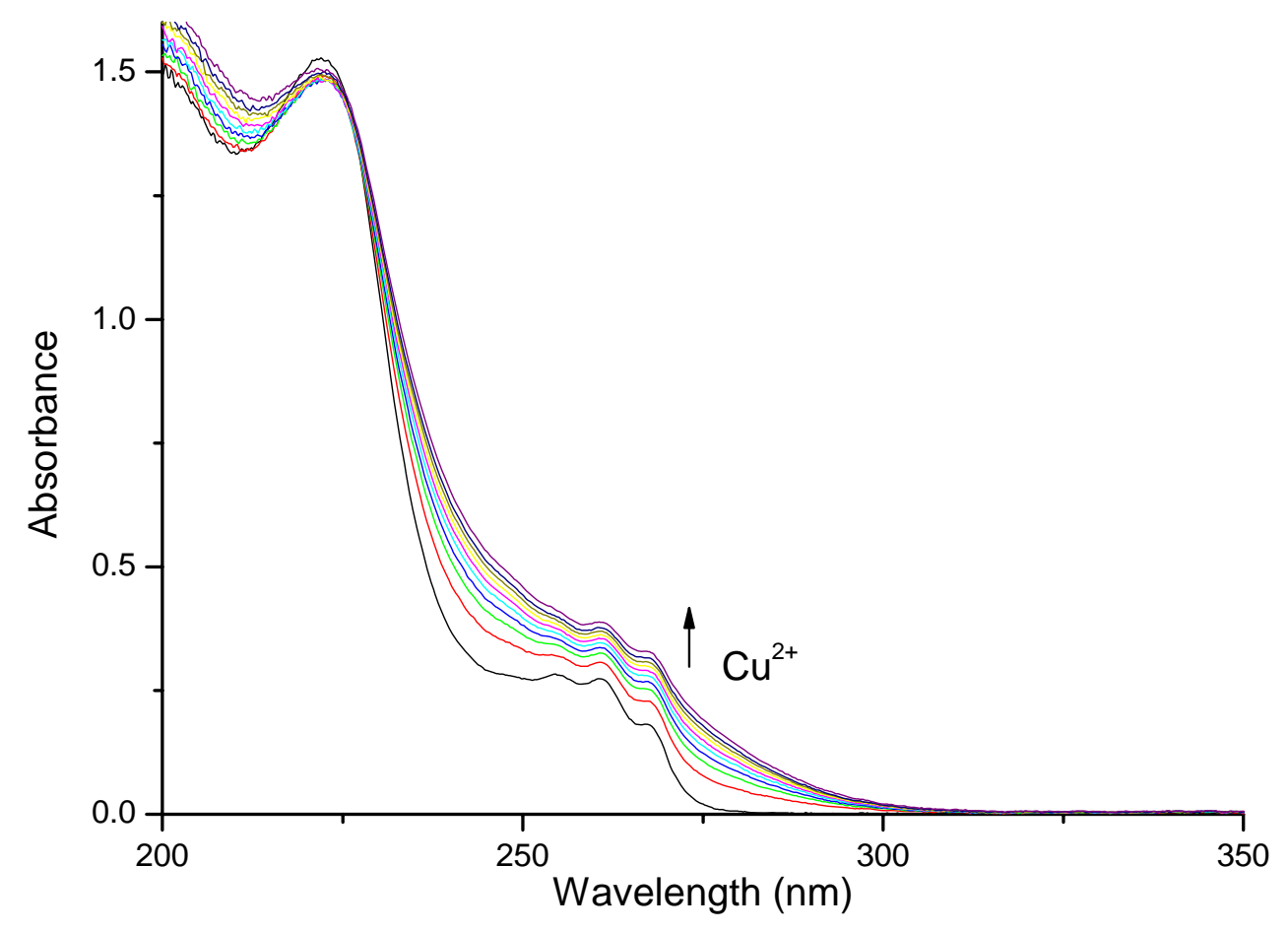

Figure S14. UV-vis titration of 12c $\left(1.909 \times 10^{-5} \mathrm{~mol} \cdot \mathrm{L}^{-1}\right)$ upon the addition of $\mathrm{CuCl}_{2}$ in acetonitrile. The concentrations of $\mathrm{CuCl}_{2}$ are $0,0.773,1.55,2.32,3.09,3.87,4.64,5.41,6.18\left(\times 10^{-5} \mathrm{~mol}^{\bullet} \mathrm{L}^{-1}\right)$, respectively.

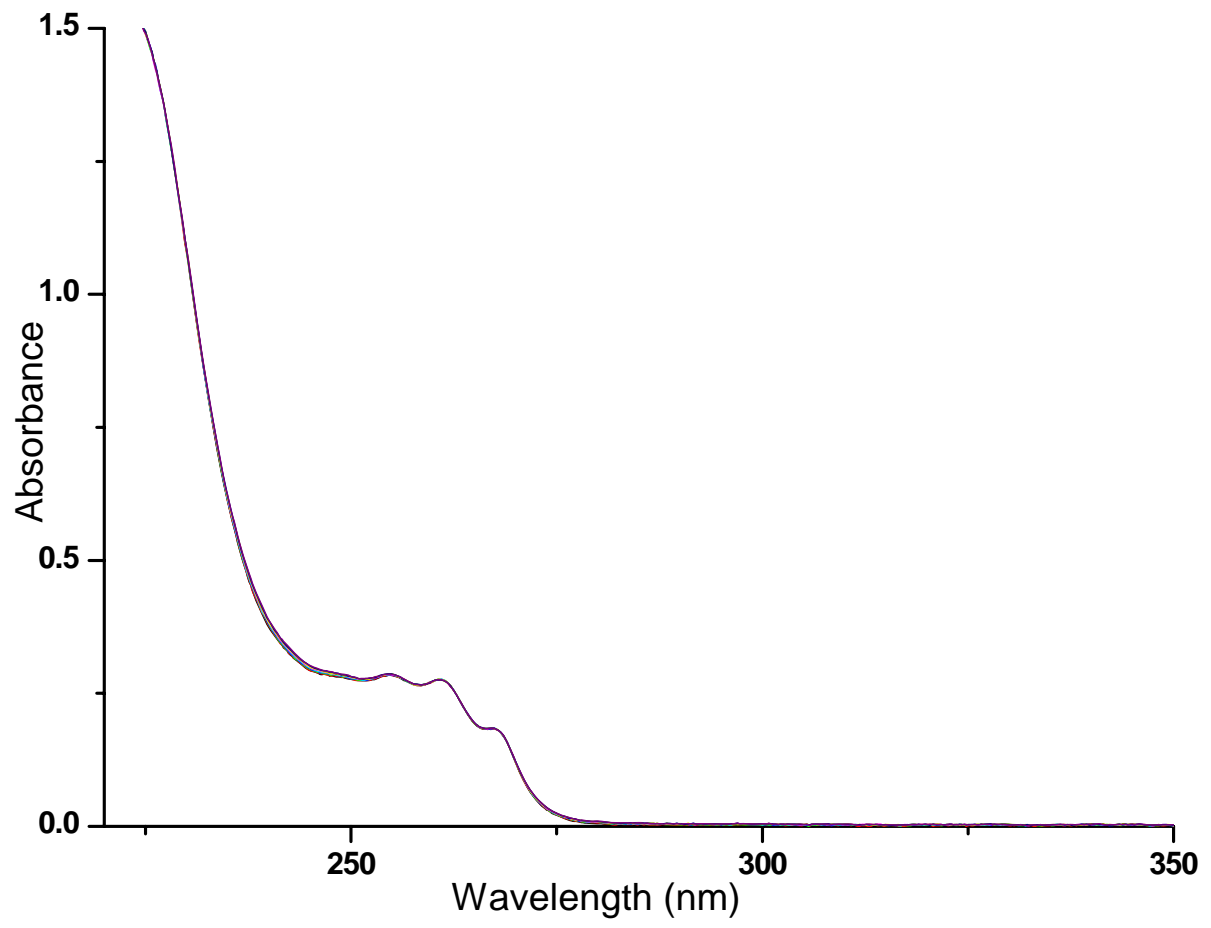

Figure S15. UV-vis titration of $12 \mathrm{c}\left(1.909 \times 10^{-5} \mathrm{~mol} \cdot \mathrm{L}^{-1}\right)$ upon the addition of $\mathrm{Fe}^{2+}$ in a mixture of acetonitrile and water $(4: 1)$. The concentrations of $\mathrm{Fe}^{2+}$ are $0,0.428,0.836,1.67,2.51,3.34,4.18,5.85$, $6.69\left(\times 10^{-5} \mathrm{~mol} \cdot \mathrm{L}^{-1}\right)$, respectively. 


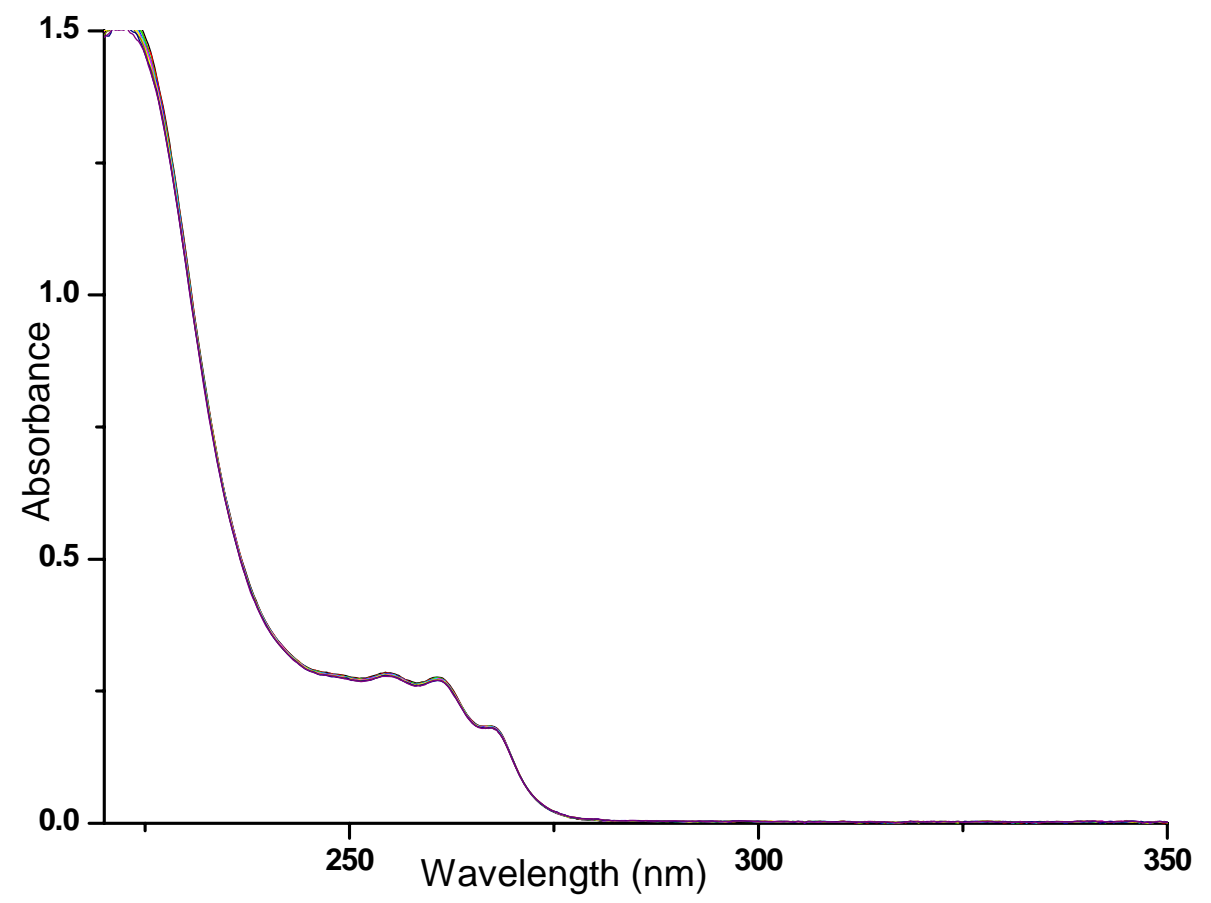

Figure S16. UV-vis titration of 12c $\left(1.909 \times 10^{-5} \mathrm{~mol} \cdot \mathrm{L}^{-1}\right)$ upon the addition of $\mathrm{Co}^{2+}$ in a mixture of acetonitrile and water $(4: 1)$. The concentrations of $\mathrm{Co}^{2+}$ are $0,0.972,1.94,2.92,3.89,4.86,5.83,6.81$, 7.78, $8.75\left(\times 10^{-5} \mathrm{~mol} \cdot \mathrm{L}^{-1}\right)$, respectively.

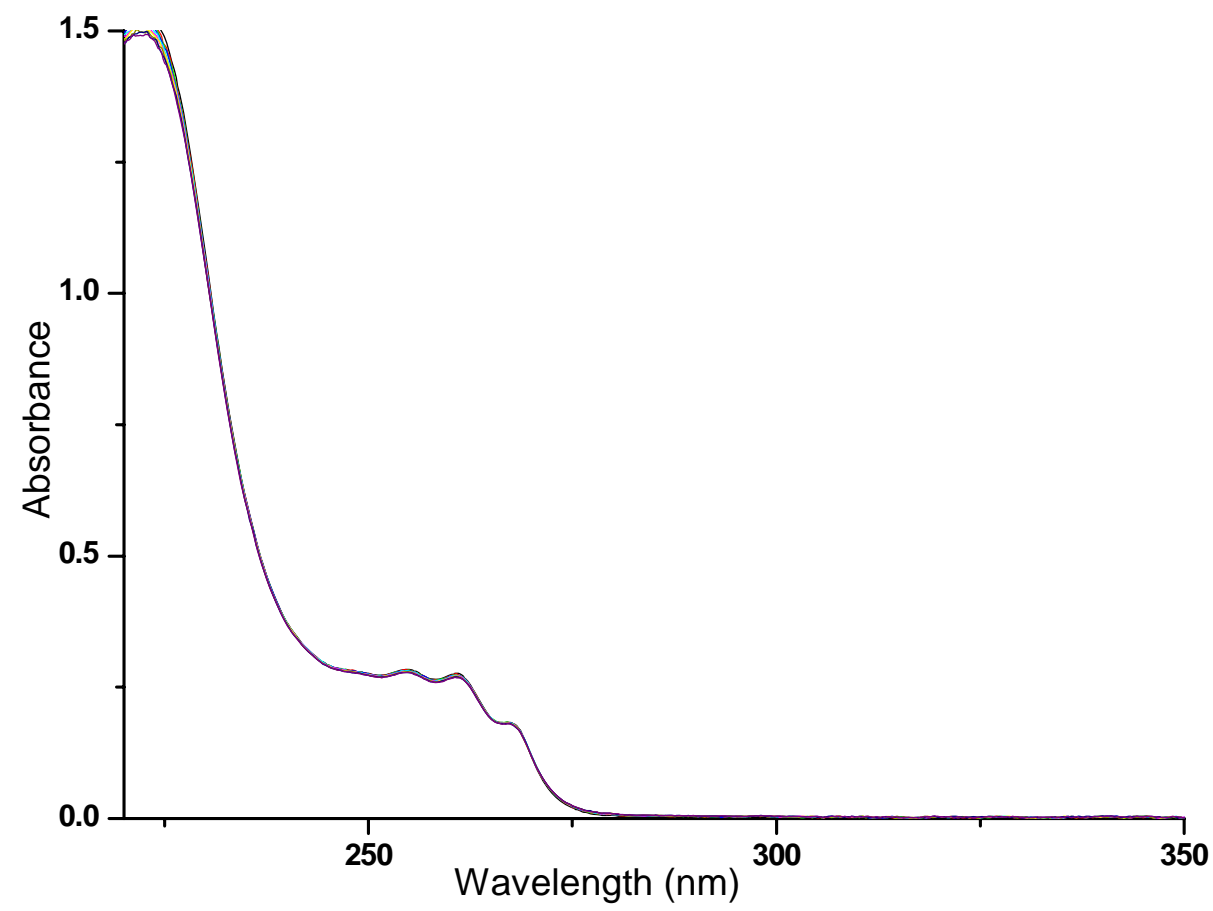

Figure S17. UV-vis titration of $12 \mathrm{c}\left(1.909 \times 10^{-5} \mathrm{~mol} \cdot \mathrm{L}^{-1}\right)$ upon the addition of $\mathrm{Ni}^{2+}$ in a mixture of acetonitrile and water $(4: 1)$. The concentrations of $\mathrm{Ni}^{2+}$ are $0,0.864,1.73,2.59,3.45,4.32,5.18,6.05$, 6.91, $7.78\left(\times 10^{-5} \mathrm{~mol} \cdot \mathrm{L}^{-1}\right)$, respectively. 


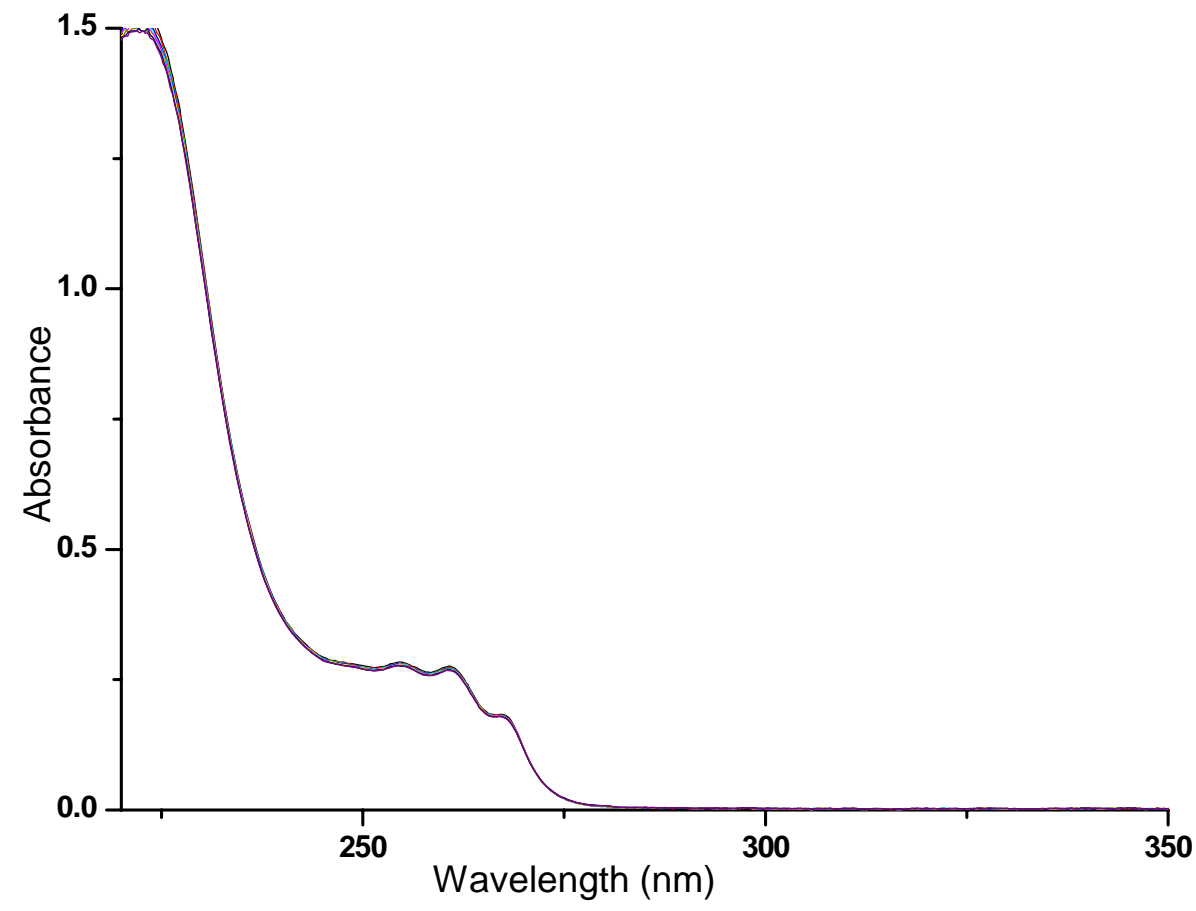

Figure S18. UV-vis titration of $12 \mathrm{c}\left(1.909 \times 10^{-5} \mathrm{~mol} \cdot \mathrm{L}^{-1}\right)$ upon the addition of $\mathrm{Zn}^{2+}$ in a mixture of acetonitrile and water $(4: 1)$. The concentrations of $\mathrm{Zn}^{2+}$ are $0,0.828,1.66,2.48,3.31,4.14,4.97,5.80$, 6.62, $7.45\left(\times 10^{-5} \mathrm{~mol} \cdot \mathrm{L}^{-1}\right)$, respectively.

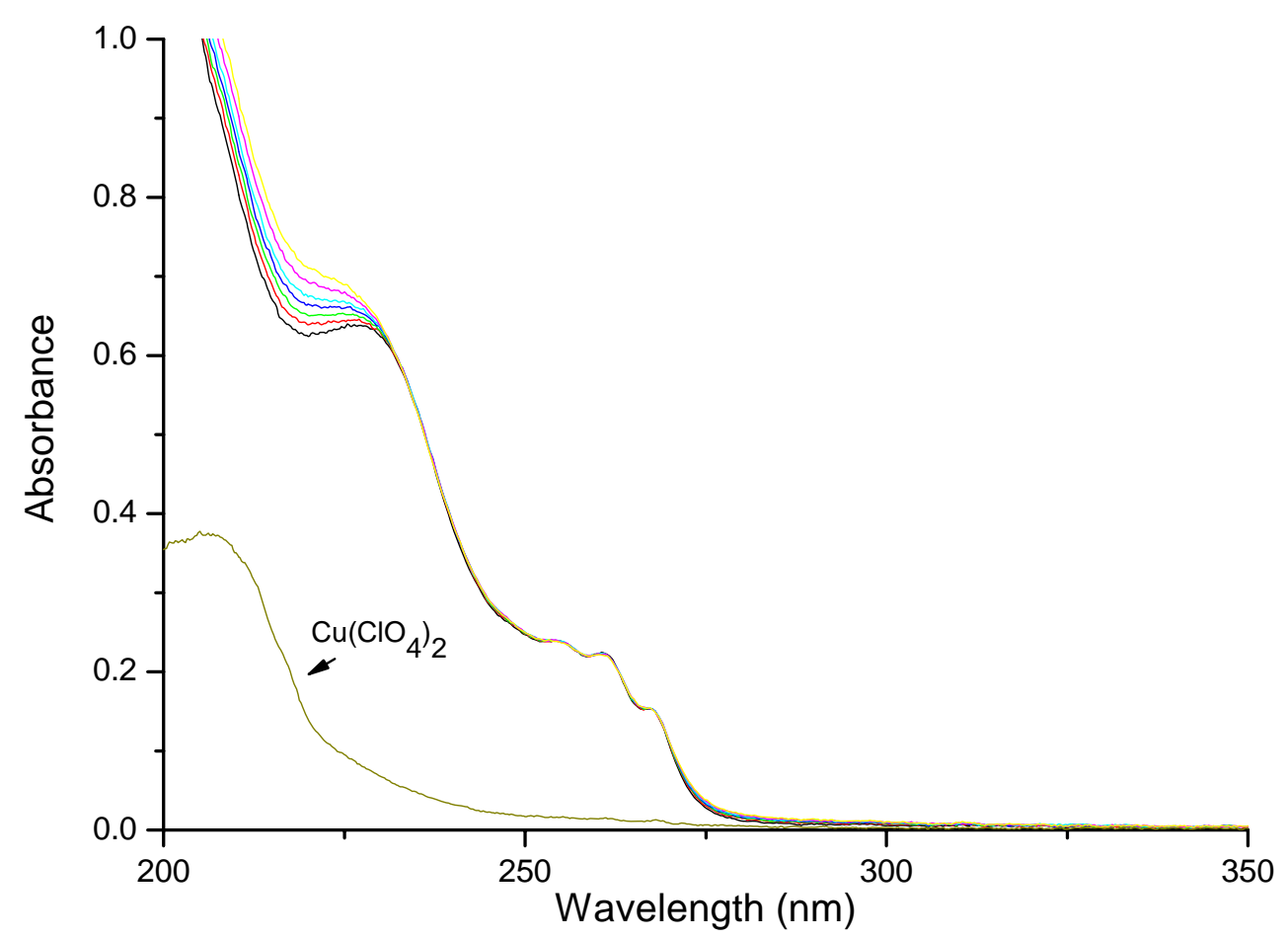

Figure S19. UV-vis titration of $13 a\left(1.66 \times 10^{-5} \mathrm{~mol} \cdot \mathrm{L}^{-1}\right)$ upon the addition of $\mathrm{Cu}^{2+}$ in a mixture of acetonitrile and water $(4: 1)$. The concentrations of $\mathrm{Cu}^{2+}$ are $0,0.257,0.514,0.771,1.03,1.54,2.06\left(\times 10^{-5}\right.$ $\left.\mathrm{mol} \cdot \mathrm{L}^{-1}\right)$, respectively. 


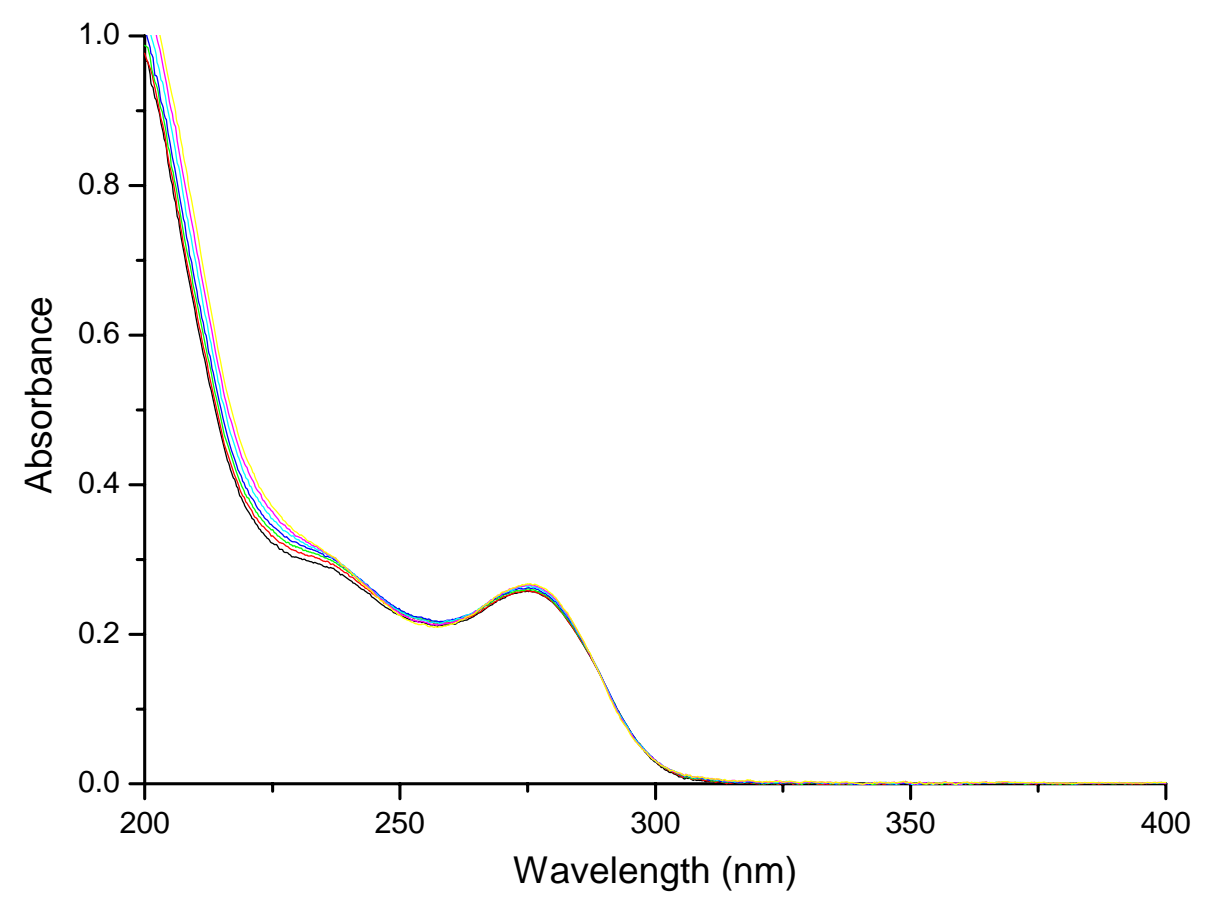

Figure S20. UV-vis titration of $\mathbf{1 3 b}\left(1.28 \times 10^{-5} \mathrm{~mol} \cdot \mathrm{L}^{-1}\right)$ upon the addition of $\mathrm{Cu}^{2+}$ in a mixture of acetonitrile and water $(4: 1)$. The concentrations of $\mathrm{Cu}^{2+}$ are $0,0.513,1.03,2.05,2.57,3.59,4.62\left(\times 10^{-5}\right.$ $\left.\mathrm{mol} \cdot \mathrm{L}^{-1}\right)$, respectively.

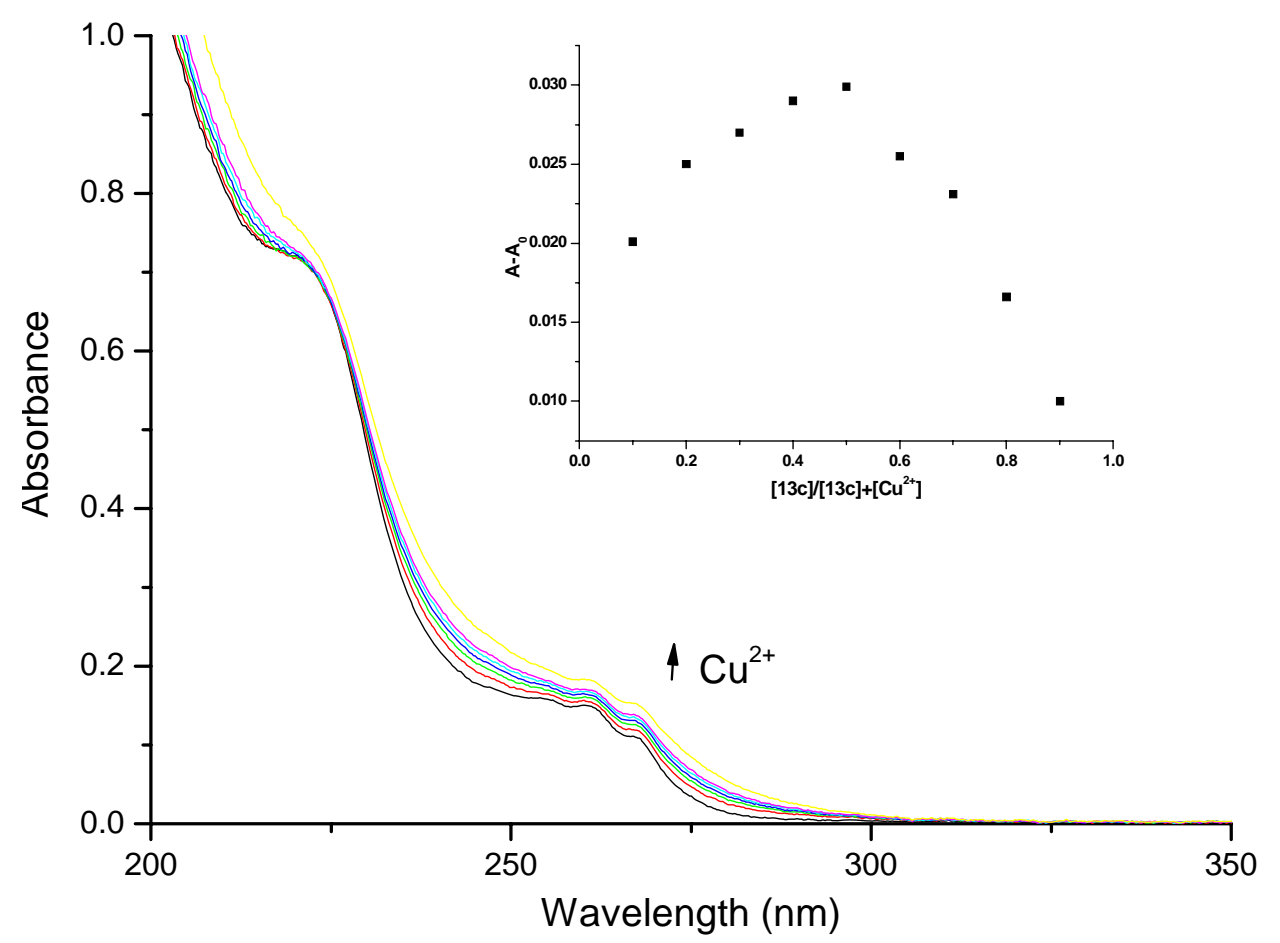

Figure S21. UV-vis titration of $13 \mathrm{c}\left(1.68 \times 10^{-5} \mathrm{~mol} \cdot \mathrm{L}^{-1}\right)$ upon the addition of $\mathrm{Cu}^{2+}$ in a mixture of acetonitrile and water $(4: 1)$. The concentrations of $\mathrm{Cu}^{2+}$ are $0,0.257,0.514,0.771,1.03,1.29,3.08\left(\times 10^{-5}\right.$ $\left.\mathrm{mol} \cdot \mathrm{L}^{-1}\right)$, respectively. The insert is the job plot of the complexation between $13 \mathbf{c}$ and $\mathrm{Cu}^{2+}$ ion in a mixture of acetonitrile and water (4:1), and the total concentration of $\mathbf{1 3 c}$ and $\mathrm{Cu}^{2+}$ is $3.20 \times 10^{-5} \mathrm{~mol}^{\circ} \mathrm{L}^{-1}$ 


\section{ESI-MS of 12a $\cdot \mathrm{Cu}\left(\mathrm{ClO}_{4}\right)_{2}$}

ESI-MS Spectrum, Y5+Cu2+

\#:1 Ret.Time:Averaged 0.400-0.853(Scan\#:16-33)

Mass Peaks:204 Base Peak:448.40(1886729) Polarity:Pos Segment1 - Event1 Intensity

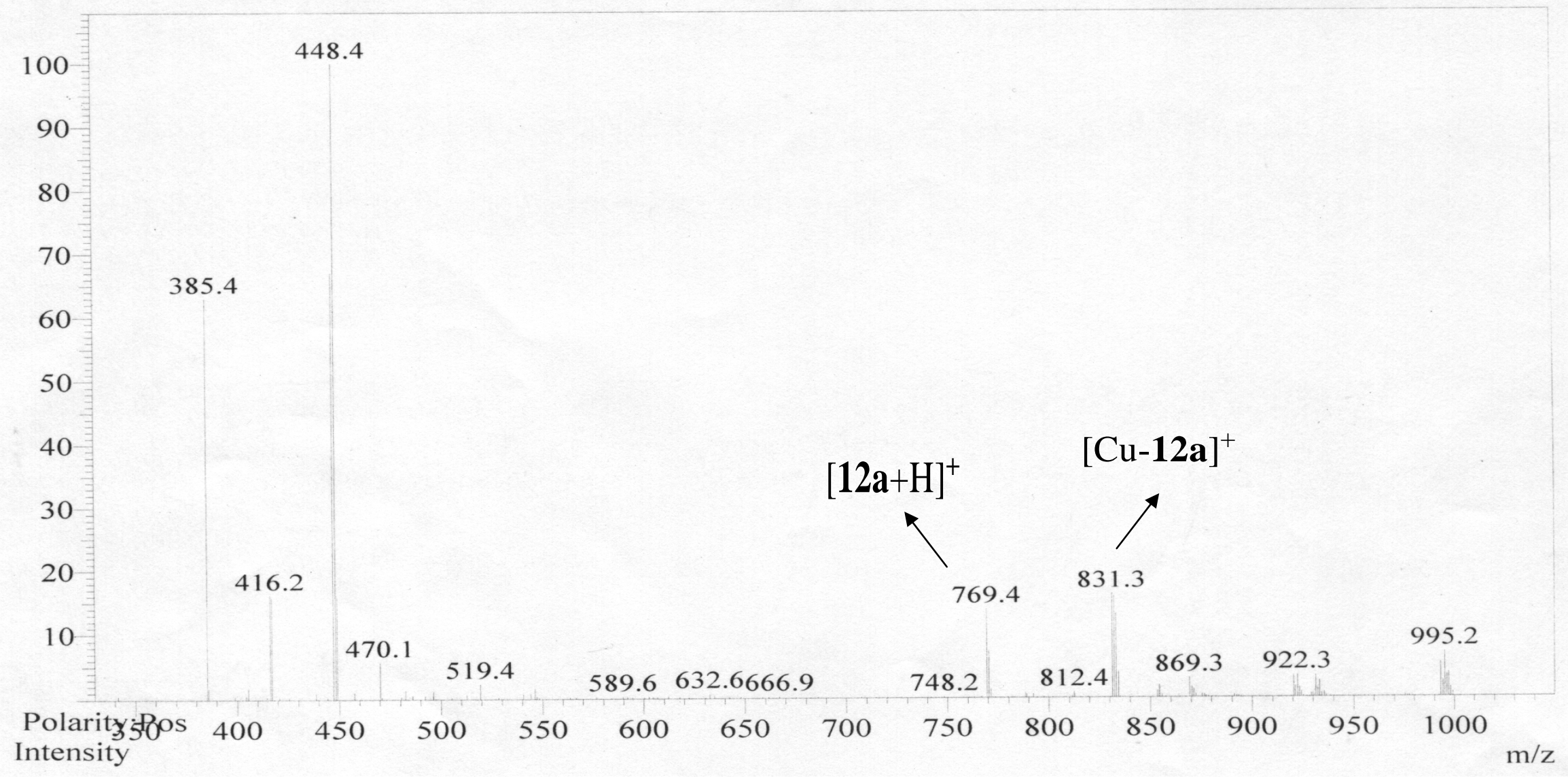




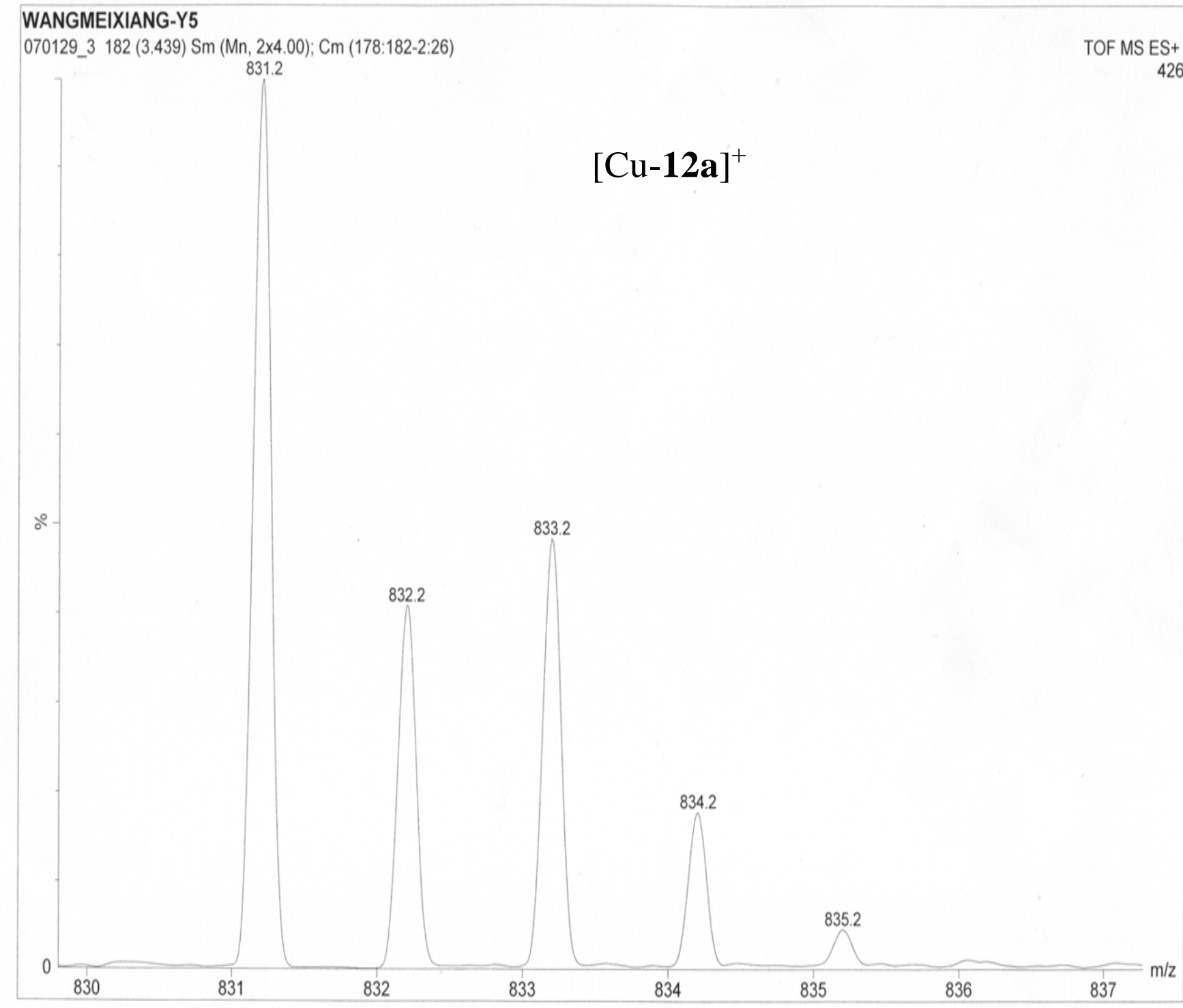

experimental

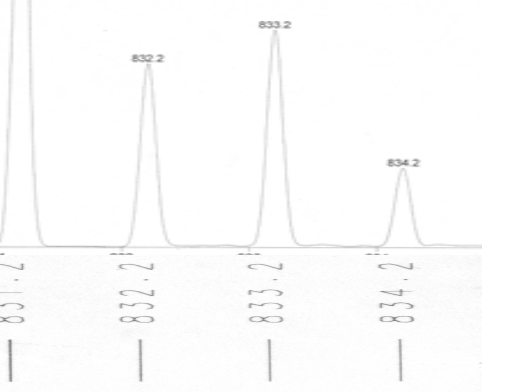

Calcd. for [Cu-12a] ${ }^{+}$

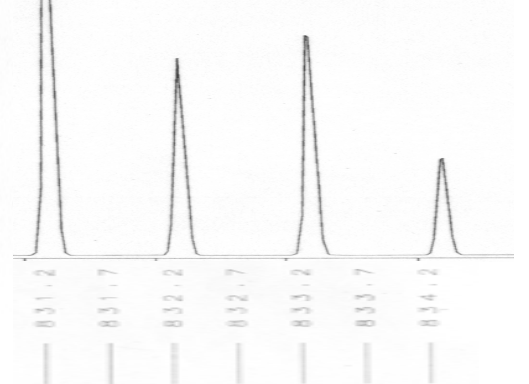

Calcd. for $\left[\mathrm{Cu}_{2}-\mathbf{1 2} \mathbf{a}_{2}\right]^{2+}$ 


\section{ESI-MS of $\mathbf{1 2 b} \cdot \mathrm{Cu}\left(\mathrm{ClO}_{4}\right)_{2}$}

ESI-MS Spectrum, Y6+Cu2+

\#:1 Ret.Time:Single 1.307(Scan\#:50)

Mass Peaks:249 Base Peak:775.85(8482210) Polarity:Pos Segment1 - Event1 Intensity

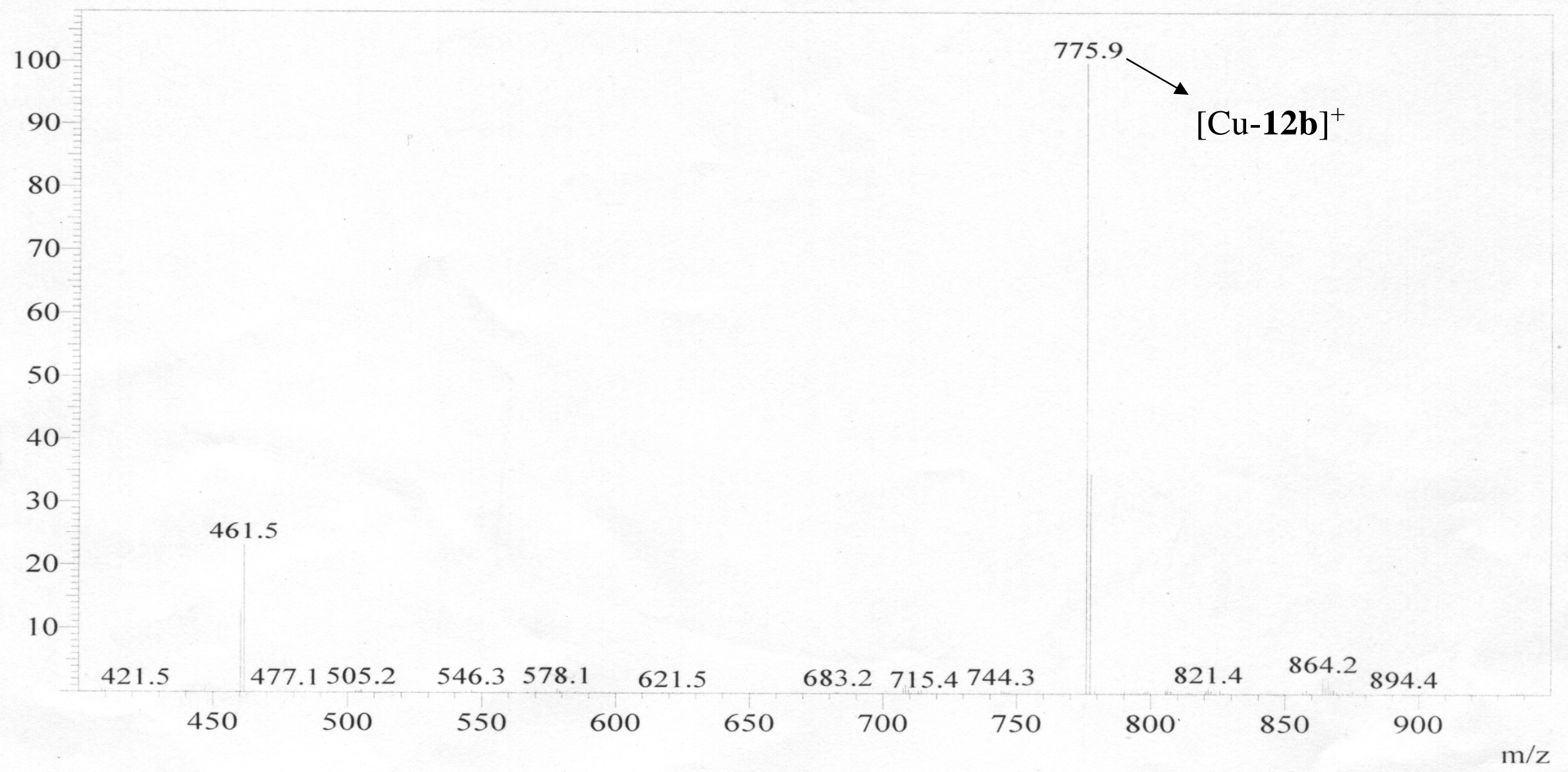




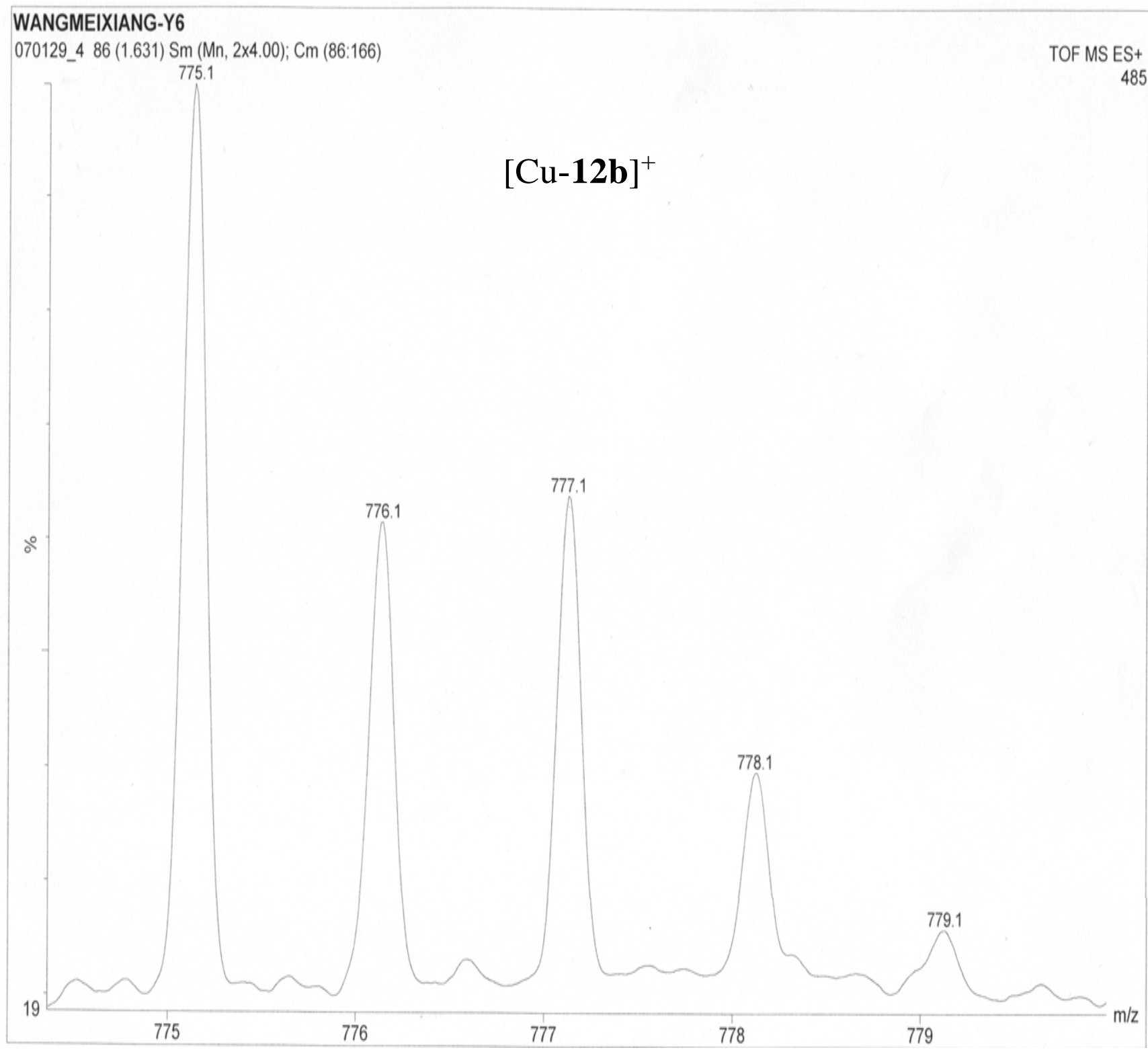

experimental

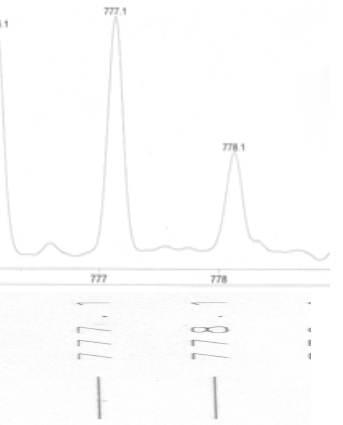

Calcd. for [Cu-12b] ${ }^{+}$

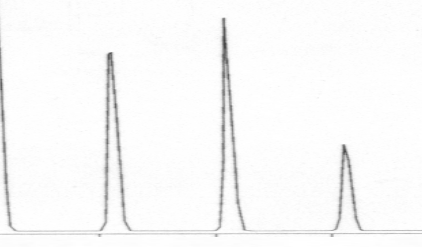

Calcd. for $\left[\mathrm{Cu}_{2}-\mathbf{1 2} \mathbf{b}_{2}\right]^{2+}$ 


\section{ESI-MS of 12c $\cdot \mathrm{Cu}\left(\mathrm{ClO}_{4}\right)_{2}$}

ESI-MS Spectrum, Y4+Cu2+

\#:1 Ret.Time:Single 1.493(Scan\#:57)

Mass Peaks:211 Base Peak:650.20(1638153) Polarity:Pos Segment1 - Event1 Intensity

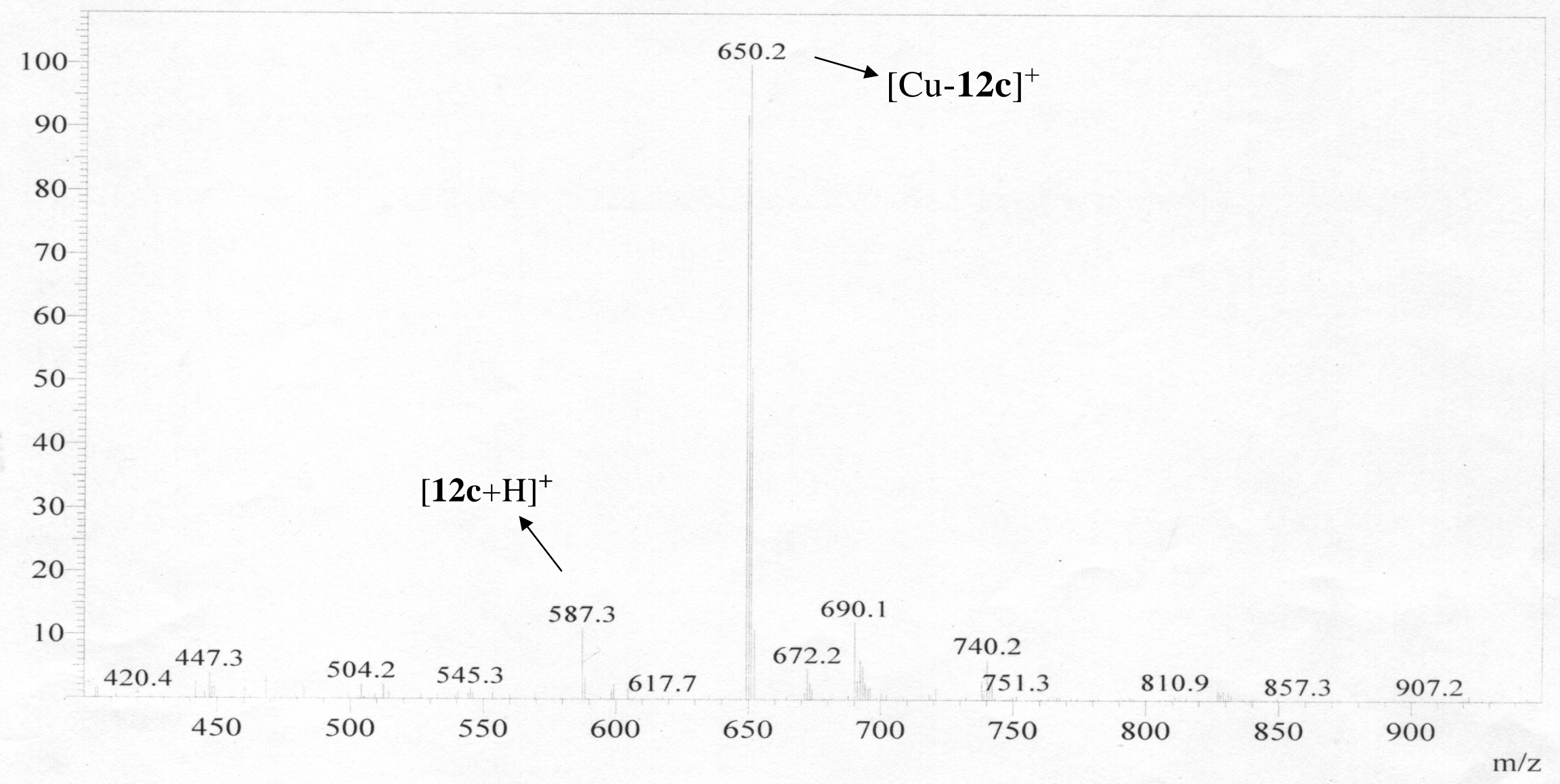




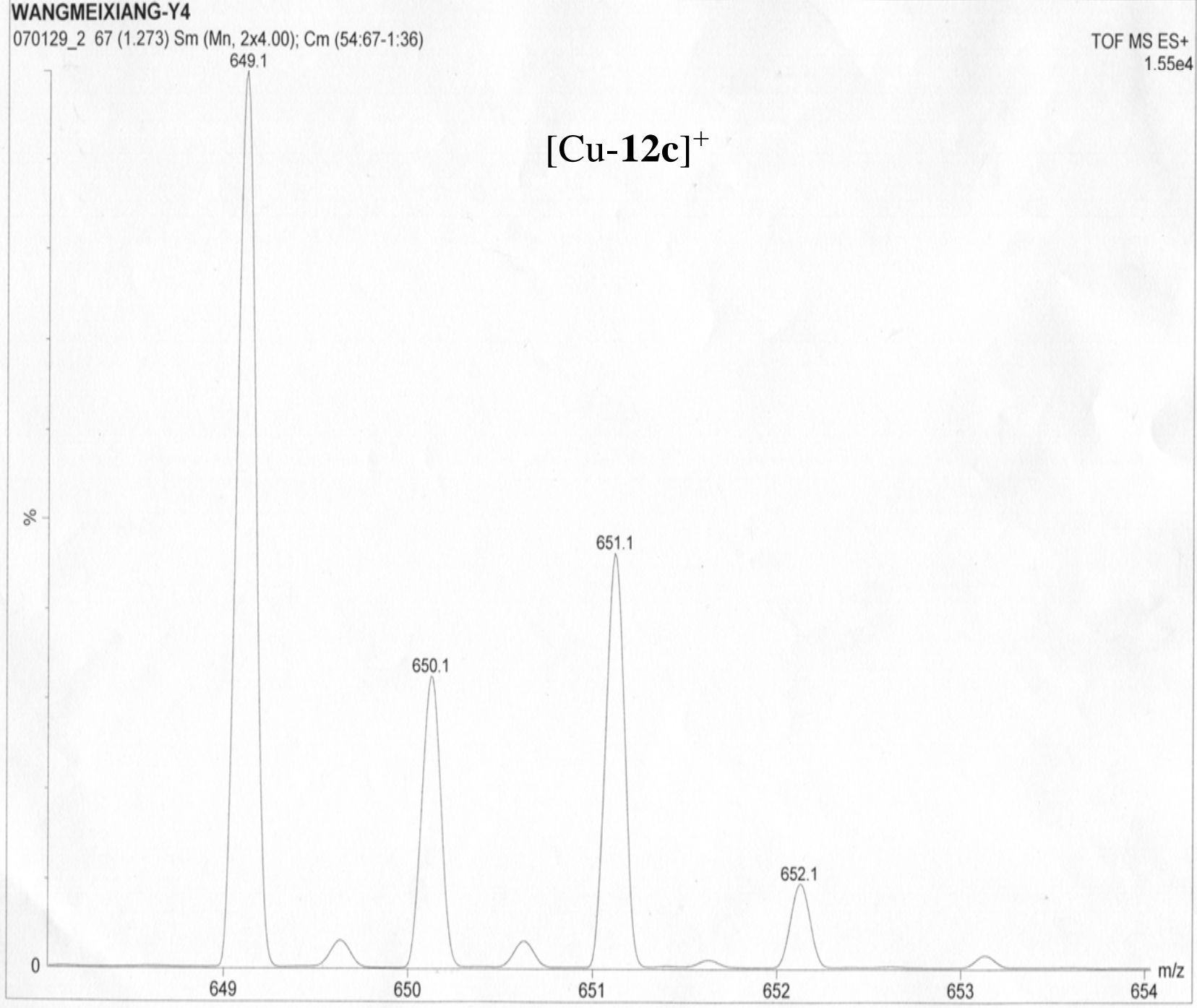

\section{experimental}

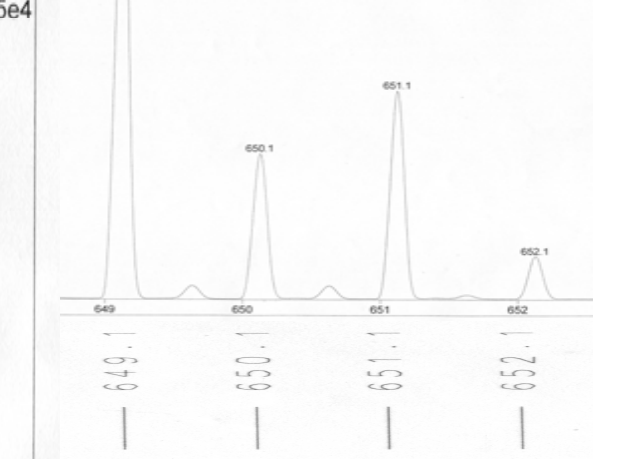

Calcd. for [Cu-12c] ${ }^{+}$

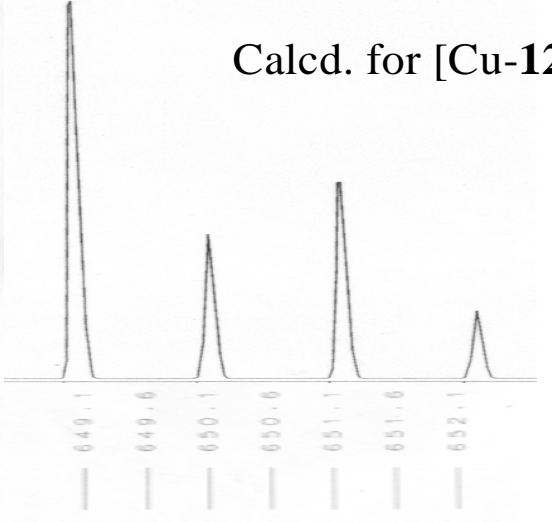

Calcd. for $\left[\mathrm{Cu}_{2}-\mathbf{1 2 c}_{2}\right]^{2+}$ 